\title{
CLER \\ NATIONAL REPORT OF FINDINGS 2019 \\ INITIAL VISITS TO SPONSORING INSTITUTIONS WITH 2 OR FEWER CORE RESIDENCY PROGRAMS
}

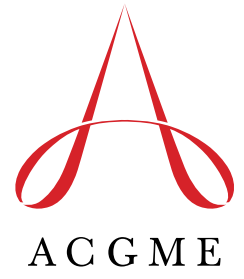

Accreditation Council for

Graduate Medical Education

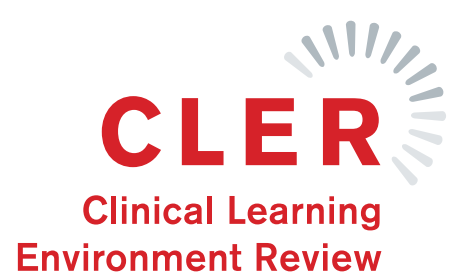

Environment Review 


\section{DEDICATION}

The Accreditation Council for Graduate Medical Education thanks the designated institutional officials at its accredited Sponsoring Institutions, as well as the executive leaders of the participating hospitals and medical centers, for graciously hosting this set of Clinical Learning Environment Review Site Visits. We appreciate the effort that went into arranging the visits and ensuring open access to residents, fellows, faculty members, and other staff. It was a privilege to spend time in your organizations, and we recognize your dedication to continually improving graduate medical education and patient care.

(C) 2019 Accreditation Council for Graduate Medical Education

ISBN: 978-1-945365-28-7

\section{SUGGESTED CITATION:}

The CLER Evaluation Committee and the CLER Program. CLER National Report of Findings 2019: Initial Visits to Sponsoring Institutions With 2 or Fewer Core Residency Programs. Chicago, IL: Accreditation Council for Graduate Medical Education; 2019: doi:10.35425/ACGME.0001 


\section{Table of Contents}

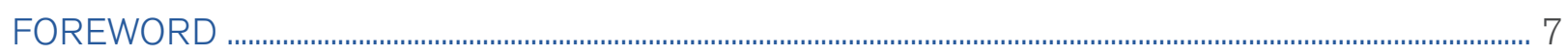

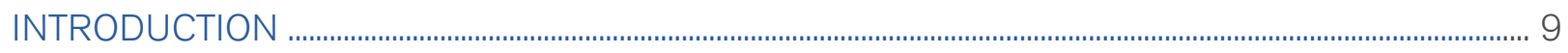

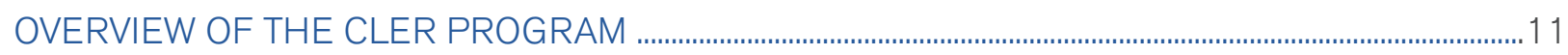

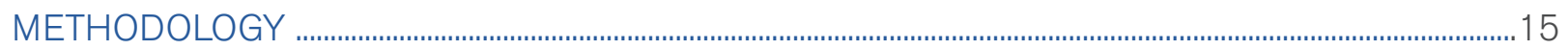

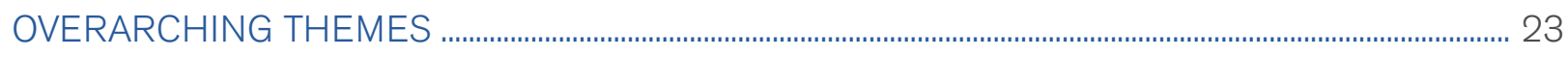

CHALLENGES AND OPPORTUNITIES IN THE 6 CLER FOCUS AREAS ….......................................... 31

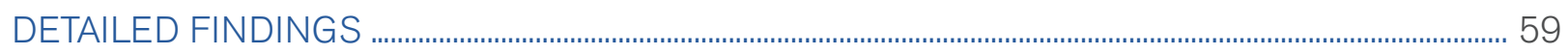

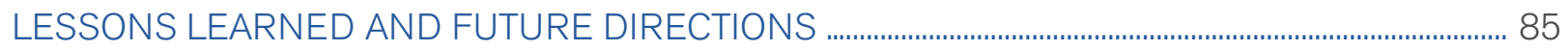

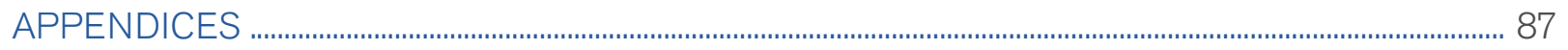




\section{Note From the CLER Evaluation Committee and the CLER Program}

This report of findings from the Clinical Learning Environment Review (CLER) Program comprises data from baseline visits to smaller Sponsoring Institutions accredited by the Accreditation Council for Graduate Medical Education. As with the 2018 report of the larger Sponsoring Institutions, this report reflects findings from CLER Protocol 2.0. Therefore, we have retained relevant and applicable information from the CLER National Report of Findings 2018 to facilitate readers' ability to easily:

- Crosswalk information in the 2018 and 2019 reports

- Understand implications of the CLER Program's findings as they relate to various clinical settings

As a result, portions of this report are reprinted and adapted from the 2018 report with permission from the Journal of Graduate Medical Education. The complete 2016 and 2018 CLER national reports are available at http://www.jgme.org.

Beginning with the next national report, we look forward to presenting all of CLER's findings-for both the smaller and larger Sponsoring Institutions-in a single biennial report. We are grateful for the graduate medical education community's ongoing support for these important efforts. 


\section{Acknowledgments}

The Accreditation Council for Graduate Medical Education (ACGME) thanks Laura Riordan, MS, for editing and overseeing the publication of this report. In addition, the ACGME acknowledges the many individuals involved in the Clinical Learning Environment Review (CLER) Program and in developing this report, including the CLER Program staff who collectively arranged and conducted the site visits, collated and analyzed the data, and provided editorial input; the members of the CLER Evaluation Committee who reviewed the results and offered their insights as to the impact of the findings; and the reviewers who generously offered their time to read early drafts and provide feedback. The ACGME thanks them for their dedication and commitment to improving graduate medical education and patient care.

(Names in alphabetical order)

\section{CLER EVALUATION}

\section{COMMITTEE MEMBERS}

John Patrick T. Co, MD, MPH, CPPS, FAAP, Co-Chair

Kevin B. Weiss, MD, Co-Chair

James P. Bagian, MD, PE*

Terry L. Cline, PhD*

Lindsay Dale, MD

David Entwistle, MHSA*

Rosemary Gibson, MSc*

Linda A. Headrick, MD, MS, FACP*

Robert Higgins, MD

Marcia Hutchinson, MD*

LCDR Dinchen Jardine, MD*

Lynne M. Kirk, MD, MACP

Anai N. Kothari, MD, MS*

Catherine M. Kuhn, MD

Tanya Lord, PhD, MPH

David Markenson, MD, MBA, FAAP, FACEP, FCCM

David Mayer, MD

Douglas E. Paull, MD, MS, FACS, FCCP, CHSE*

Lakshmana Swamy, MD, MBA*
Andrew M. Thomas, MD, MBA*

Marjorie S. Wiggins, DNP, MBA, RN, FAAN, NEA-BC

Ronald Wyatt, MD, MHA, DMS(Hon)

\section{CLER PROGRAM STAFF}

Mark R. Bixby, MD, FAAFP

Isabelle Bourgeois, MPA

Jennifer J. Buescher, MD, MSPH

Robert Casanova, MD, MHPE

Baretta R. Casey, MD, MPH, FAAFP ${ }^{\star \star}$

Marian D. Damewood, MD, FACOG

Kevin C. Dellsperger, MD, PhD

Robin Dibner, MD

David L. Dull, MD, MMM, FAAPL

Staci A. Fischer, MD

Octavia Franklin

Patrick Guthrie

Paula Hensley, MPH

Kristen Ward Hirsch

John A. Hopper, MD 
Sharhabeel Jwayyed, MD, MS

Catherine Kallal, MD**

Elizabeth Kimball, MA**

Nancy J. Koh, PhD

Kathryn E. McGoldrick, MD, MAH, FCAI(Hon)

Clifton McReynolds, PhD

Terrie Mendelson, $\mathrm{MD}^{\star \star}$

Joshua Mirôn, MA

Robin C. Newton, MD, FACP

Morgan Passiment, MS

Douglas E. Paull, MD, MS, FACS, FCCP

Daniel Picard, MD**

Kathy B. Porter, MD, MBA, FACOG

Dale Ray, MD, MMM

Laura Riordan, MS

Melissa Schori, MD, FACP, MBA

Tara Shedor, MA

Stephen Smith, MD**

Mike Strickland, MFA**

Hongling Sun, $\mathrm{PhD}$

Marie Trontell, MD

Paul Uhlig, MD, MPA

Robin Wagner, RN, MHSA

Elizabeth Wedemeyer, MD

Kevin B. Weiss, MD

Esther Woods

Martha S. Wright, MD, MEd

James R. Zaidan, MD, MBA

Jose Zayas, DO, FAAP
OTHER ACGME STAFF

Kara Etolen

Paul Foster Johnson, MFA

Olivia Orndorff, MSLIS

Cassie Pritchard, MPP

\section{REVIEWERS}

Paige Amidon, MBA, MPH

John Patrick T. Co, MD, MPH, CPPS, FAAP

John Combes, MD

John Duval, MBA

David Entwistle, MHSA

Jeffrey P. Gold, MD

Donald Goldmann, MD

Diane M. Hartmann, MD

Eric Holmboe, MD, FACP, FRCP

Susan Kirk, MD

Betsy Lee, RN, BSN, MSPH

Thomas J. Nasca, MD, MACP

Ingrid Philibert, PhD, MBA

Rowen K. Zetterman, MD, MACP, MACG

*Past CLER Evaluation Committee member

${ }^{\star *}$ Former staff member 


\section{Foreword}

Jeffrey P. Gold, MD, Chair, ACGME Board of Directors

The Accreditation Council for Graduate Medical Education (ACGME) Board of Directors is continually seeking to understand how best to serve the public in its mission to improve health care through accreditation. To help deliver on this mission, the ACGME's Board of Directors established the Clinical Learning Environment Review (CLER) Program to provide formative feedback in 6 focus areas ${ }^{1}$ to accredited Sponsoring Institutions (SIs) on the quality of their clinical learning environments (CLEs) for graduate medical education (GME). Through its national reports, the CLER Program contributes to the ACGME's understanding of the state of our nation's CLEs by providing new knowledge that can inform the ACGME's efforts to continually improve the quality of GME.

The first 2 CLER national reports, which focused on larger Sls (ie, those with 3 or more core residency programs), 2,3 provided an important baseline to the ACGME Board, the GME community, and the public, with insights into the quality of CLEs. Findings from these reports demonstrated a high degree of variability and, often, opportunity to improve how residents and fellows were exposed to basic experiences in patient safety and health care quality during their clinical training. These insights helped shape the recent revisions of the ACGME Common Program Requirements. ${ }^{4}$ The CLER Program's findings are also helping shape understanding of the nature of resident, fellow, and faculty fatigue and burnout. These important elements relate to the key issue of well-being that the ACGME is seeking to address directly and through national collaborative partnerships. ${ }^{5}$

The present report focuses on findings for visits to 270 ACGME-accredited smaller SIs (ie, those with 2 or fewer core residency programs). These Sls represent an important part of the GME community, as many of their CLEs are rural, smaller health care organizations with a focus on ambulatory care-based clinical training. Notable to this report is how these smaller Sls are generally facing the same challenges and opportunities as the larger Sls in creating and maintaining high-performing CLEs.

Findings from this CLER national report reveal a large degree of variation in how CLEs engage residents and fellows in patient safety, health care quality, and the other CLER Focus Areas. This variation was noted both within and between CLEs. This report also highlights positive findings for important aspects of the environment, such as residents' and fellows' high degree of confidence that there is appropriate supervision. Similar to the reports on larger SIs, this report identifies the importance of the clinical team and how team members-specifically nurses-can help ensure high-quality supervision and thereby improve care.
These SIs represent an important part of the GME community, as many of their CLEs are rural, smaller health care organizations with a focus on ambulatory care-based clinical training. Notable to this report is how these smaller Sis are generally facing the same challenges and opportunities as the larger SIs in creating and maintaining high-performing CLEs. 
With the publication of this baseline report of CLER findings focusing on smaller Sls, the ACGME and its Board of Directors, the GME community, and the public have a way of better understanding and improving our nation's CLEs. We look forward to the ongoing work of the CLER Program with its evolving focus on well-being and team-based initiatives. We remain excited to see how this formative evaluation process is serving as an instrument to create a new national conversation on improving the CLEs for all ACGMEaccredited GME programs. Better GME . . . better patient care!

\section{REFERENCES}

1. Weiss KB, Bagian JP, Wagner R. CLER Pathways to Excellence: expectations for an optimal clinical learning environment [executive summary]. J Grad Med Educ. 2014;6(3):610-611. doi:10.4300/JGME-D-14-00348.1

2. Wagner R, Koh NJ, Patow C, Newton R, Casey BR, Weiss KB. Detailed findings from the CLER National Report of Findings 2016. J Grad Med Educ. 2016;8(2s1):35-54. doi:10.4300/1949-8349.8.2s1.35

3. Koh NJ, Wagner R, Newton R, et al. Detailed findings from the CLER National Report of Findings 2018. J Grad Med Educ. 2018;10(4s):49-68. doi:10.4300/1949-8349.8.2s1.35

4. Accreditation Council for Graduate Medical Education. Common Program Requirements. Chicago, IL; 2017. https://www. acgme.org/Portals/O/PFAssets/ProgramRequirements/CPRs_2017-07-01.pdf.

5. Brigham T, Barden C, Legreid Dopp A, et al. A Journey to Construct an All-Encompassing Conceptual Model of Factors Affecting Clinician Well-Being and Resilience. NAM Perspectives Discussion Paper. Washington, DC: National Academy of Medicine; 2018. doi:10.31478/201801b 


\section{Introduction}

Since implementing the Clinical Learning Environment Review (CLER) Program more than 7 years ago, the Accreditation Council for Graduate Medical Education (ACGME) and the nearly 800 ACGME-accredited Sponsoring Institutions (SIs) have rapidly gained new knowledge about the clinical environments that host graduate medical education (GME). By periodically visiting ACGME-accredited Sls and providing formative feedback, the CLER Program has served to heighten awareness about the large degree of variability in how residents and fellows engage in learning related to the 6 CLER Focus Areas of patient safety; health care quality and reduction in health care disparities; care transitions; supervision; fatigue management, mitigation, and duty hours; and professionalism.

The CLER Program has provided key insights that have informed recent revisions to the ACGME Common Program Requirements. ${ }^{1}$ While aggregate information from the CLER Program will continue to inform future accreditation standards, the CLER Program will also continue to remain a formative evaluation for Sls, completely separate from institutional accreditation. As such, the CLER Program is designed to provide Sls with an objective assessment by which they can better collaborate with the leadership of their clinical care partners to advance both excellence in patient care and resident and fellow learning.

The CLER National Report of Findings 2019: Initial Visits to Sponsoring Institutions With 2 or Fewer Core Residency Programs includes a new set of insights into the clinical learning environments (CLEs) associated with ACGMEaccredited Sls that have 2 or fewer core programs. The overarching themes of this report are to a large degree consistent with prior reports of the larger Sls with more than 2 core programs. ${ }^{2}$ In addition, the present findings reveal that CLEs affiliated with smaller Sls have many of the same challenges and opportunities across the 6 CLER Focus Areas as CLEs of larger Sls. ${ }^{3}$ For example, resident and fellow recognition and reporting of patient safety events into the clinical site's patient safety event reporting system varied across CLEs. In addition, CLEs, regardless of size, appear to have similar challenges with regard to GME engagement in transitions of care and professionalism.

The Sls represented in this report disproportionally represent the CLEs associated with medically underserved populations and rural areas. Similarly, this report disproportionately represents the CLEs associated with federally qualified health centers and teaching health centers sponsored by the Health Resources \& Services Administration.

Over the past 2 years, the ACGME has increased its efforts to better understand the medically underserved areas in the United States and the populations in these areas that are in need of physicians and other health care providers. Based on the work of a large multi-stakeholder task force, the ACGME Board of Directors recently approved a new initiative that will explore ways to support the growth of new Sls and residency
This CLER National Report and those that follow will support the ACGME's ability to best understand the needs of SIs and their CLEs for this important segment of the population, with a dual goal of supporting the expansion of GME programs in rural and underserved areas to promote access and quality, while upholding the single high standards of quality of education associated with ACGME accreditation. 
and fellowship training programs in these underserved areas. This CLER National Report and those that follow will support the ACGME's ability to best understand the needs of Sls and their CLEs for this important segment of the population, with a dual goal of supporting the expansion of GME programs in rural and underserved areas to promote access and quality, while upholding the single high standards of quality of education associated with ACGME accreditation.

This report also provides national data from ACGME that reinforces how widespread the issues of burnout are among faculty members and program directors. For example, this report finds that, in nearly half of the CLEs, residents and fellows noted that they have observed some signs of burnout among faculty members and program directors. It is possible that the CLEs associated with these smaller SIs are facing special issues-as their small size means they have less total faculty available for coverage and backup leading to less opportunity to decompress from stressful workloads or take time for personal and family responsibilities.

It is essential that the ACGME continue to explore and address issues related to well-being, including burnout. In partnership with the Association of American Medical Colleges, the National Academy of Medicine, and many other organizations, the ACGME is part of a national community of learning focused on improving the wellbeing of physicians and other members of the health care workforce. The data from the CLER Program provide continued impetus for this collaboration.

In its current protocol, the CLER Program is assisting the ACGME and the GME community in gaining a deeper understanding of how well-being is addressed in our nation's CLEs. Over time, CLER data may help to assess progress in promoting well-being and addressing the disquieting level of burnout among the talented individuals who serve as GME faculty and program directors and therefore role models for residents and fellows.

Through the CLER Program's Pursuing Excellence in Clinical Learning Environments initiative, the ACGME continues to work with the GME community to acquire new knowledge and share successful models and approaches to addressing the challenges in the CLER Focus Areas. All of the Pursuing Excellence learning collaboratives to date include teams from smaller Sls. Together, the teams participating in these collaboratives are both learning from each other and inspiring new ideas on how to improve the CLE.

On behalf of ACGME, I hope that this report stimulates new discussions among the clinical and educational leadership of the hospitals, health centers, and ambulatory care sites that serve as CLEs and comprise an essential part of the US health care environment. I look forward to seeing how, over time, this information serves as a formative instrument for improving both GME and patient care.

\section{REFERENCES}

1. Accreditation Council for Graduate Medical Education. Common Program Requirements. Chicago, IL; 2017. https://www. acgme.org/Portals/O/PFAssets/ProgramRequirements/CPRs_2017-07-01.pdf.

2. Co JPT, Bagian JP, Weiss KB, on behalf of the CLER Evaluation Committee. The overarching themes from the CLER National Report of Findings 2018. J Grad Med Educ. 2018;(August):19-24. http://www.jgme.org/doi/pdf/10.4300/19498349.10.4s.49.

3. Weiss KB, Co JPT, Bagian JP, on behalf of the CLER Evaluation Committee. Challenges and opportunities in the six focus areas: CLER National Report of Findings 2018. J Grad Med Educ. 2018;10(4S):25-48. doi:10.4300/1949-8349.8.2s1.25 


\section{Overview of the CLER Program}

Robin Wagner, RN, MHSA; Baretta R. Casey, MD, MPH, FAAFP; Robin C. Newton, MD, FACP; Nancy J. Koh, PhD; and Kevin B. Weiss, MD; on behalf of the CLER Program*

\section{INTRODUCTION}

Since its inception in 2012, the Clinical Learning Environment Review (CLER) Program has had at its core a commitment to formative assessment and feedback regarding graduate medical education (GME) engagement in 6 important, cross-cutting areas of focus-patient safety; health care quality; care transitions; supervision; fatigue management, mitigation, and duty hours; and professionalism. By conducting periodic site visits and providing formative feedback to hospitals, medical centers, and ambulatory care sites that serve as clinical learning environments (CLEs) for resident and fellow physicians, the CLER Program aims to stimulate conversations and motivate CLEs to build upon their strengths and internally address opportunities for improvement.

The CLER Program refers to CLEs as living and breathing entities-the embodiment of all of the individuals within these settings that influence and imprint upon residents, fellows, and other early learners. Through its formative approach, CLER recognizes that, although there are shared elements among CLEs, each has a unique set of internal and external factors that influence the development and implementation of that CLE's strategic goals aimed at improving patient care.

The CLER Program's primary link to accreditation is that every Sponsoring Institution (SI) accredited by the Accreditation Council for Graduate Medical Education must periodically complete a CLER Site Visit. The present report includes findings from the first set of CLER visits to 270 participating sites of smaller Sls (ie, those with 2 or fewer core residency programs).

Of note, CLER visit findings from the smallest sites of the Sls visited (ie, preventive medicine programs and single fellowship programs) are not included in this national report. A separate report will be published in the near future on the data from these sites.

\section{THE CLER SITE VISIT PROCESS AS ADAPTED FOR SMALLER SPONSORING INSTITUTIONS}

For the CLER Site Visits that informed the present report, the CLER Program modified its site visit process to accommodate the unique features of the smaller Sls. The following text highlights these modifications and provides a brief overview of the protocol for the CLER Site Visit. For a more detailed description of the protocol, please see the Methodology section of this report (pp 15-22). 
For each site visit, CLER Site Visitors met with the CLE's GME and executive leadership and leaders in patient safety and health care quality; held group interview sessions with residents and fellows, faculty physicians, and program directors; and had numerous conversations with various members of the clinical care team while on walking rounds within the CLE. At the end of the visit, the CLER Site Visitors provided GME and executive leadership with an oral report that synthesized the team's findings in the 6 CLER Focus Areas. After the visit, the CLER Program provided the CLE with a more detailed written report of the site visit findings.

While the overall site visit structure and protocol were nearly identical to those used on visits to the larger Sls, ${ }^{1,2}$ they were modified slightly in the following ways on an as-needed basis to accommodate some of the unique features of these CLEs:

- The site visit agenda was modified as needed to allow for travel between the inpatient site and the main ambulatory care clinical site.

- For a small proportion of the Sls, the visit was held exclusively in the ambulatory care setting. These included visits to Sls that are teaching health centers administered by the Health Resources \& Services Administration and, in a few circumstances, visits to Sls where the major participating site for the inpatient setting had already received a CLER Site Visit under a different SI.

- For the small proportion of Sls where the visit was exclusive to the ambulatory setting, the protocol questions and scenarios were modified to fit the setting while keeping the essence of the questions to allow for comparability across settings.

- In Sls with 1 residency or fellowship program, if the program director was also the designated institutional official, the program director attended the executive leadership meetings and assigned a designee (often an associate program director) for the program director interview session.

- In Sls with 1 or 2 program directors, while the interview sessions for the program directors were separate from the sessions with faculty members, the results were combined in the verbal and written reports to maintain anonymity.

\section{THE CLER EVALUATION COMMITTEE}

From the inception of the CLER Program, the CLER Evaluation Committee has provided oversight of and guidance for all aspects of program development. The committee is composed of members with expertise in patient safety and health care quality improvement, as well as GME and executive leadership of hospitals and medical centers (eg, chief medical officer, chief nursing officer). The committee also includes postgraduate physician representation and public members.

For this report, the committee reviewed the data from the smaller SI site visits and brought an external voice in response to the findings-presented in the sections on overarching themes and challenges and opportunities in the CLER Focus Areas. Their views and commentary on the significance of the overarching themes and the challenges and opportunities are reflected in the discussion portions of these sections. 


\section{REPORTING THE FINDINGS AND ORGANIZATION OF THE REPORT}

In the following sections, CLER Program staff and the CLER Evaluation Committee present the findings and their implications from several different perspectives, including overarching themes, highlights of the challenges and opportunities in each of the 6 CLER Focus Areas, and detailed findings. The appendices of this report (pp 87-130) contain additional information about the Sls visited, demographic information, and graphic displays of selected data.

\section{REFERENCES}

1. Koh NJ, Wagner R, Weiss KB, on behalf of the CLER Program. The methodology for the CLER National Report of Findings 2016. J Grad Med Educ. 2016;8(2s1):15-19. doi:10.4300/1949-8349.8.2s1.15

2. Koh NJ, Wagner R, Sun H, Weiss KB, on behalf of the CLER Program. The methodology for the CLER National Report of Findings 2018. J Grad Med Educ. 2018;10(4s):13-18. doi:10.4300/1949-8349.8.2s1.15

\section{${ }^{*}$ CLER PROGRAM}

Mark R. Bixby, MD, FAAFP; Isabelle Bourgeois, MPA; Jennifer J. Buescher, MD, MSPH; Robert Casanova, MD, MHPE;

Baretta R. Casey, MD, MPH, FAAFP**; Marian D. Damewood, MD, FACOG; Kevin C. Dellsperger, MD, PhD; Robin Dibner, MD;

Staci A. Fischer, MD; Paula Hensley, MPH; John A. Hopper, MD; Sharhabeel Jwayyed, MD, MS; Catherine Kallal, MD**;

Elizabeth Kimball, MA**; Nancy J. Koh, PhD; Kathryn E. McGoldrick, MD, MAH, FCAl(Hon); Clifton McReynolds, PhD;

Terrie Mendelson, MD**; Joshua Mirôn, MA; Robin C. Newton, MD, FACP; Daniel Picard, MD**; Kathy B. Porter, MD, MBA, FACOG;

Dale Ray, MD, MMM; Laura Riordan, MS; Melissa Schori, MD, FACP, MBA; Stephen Smith, MD**; Mike Strickland, MFA**; Hongling Sun, PhD; Marie Trontell, MD; Paul Uhlig, MD, MPA; Robin Wagner, RN, MHSA; Elizabeth Wedemeyer, MD; Kevin B. Weiss, MD; Esther Woods; Martha S. Wright, MD, MEd; James R. Zaidan, MD, MBA; Jose Zayas, DO, FAAP

${ }^{\star \star}$ Former staff member 
$14 \mid$ OVERVIEW | CLER National Report of Findings 2019 


\section{Methodology}

Nancy J. Koh, PhD; Robin Wagner, RN, MHSA; Baretta R. Casey, MD, MPH, FAAFP; Hongling Sun, PhD; and Kevin B. Weiss, MD; on behalf of the CLER Program*

\section{INTRODUCTION}

The Accreditation Council for Graduate Medical Education (ACGME) established the Clinical Learning Environment Review (CLER) Program ${ }^{1,2}$ to provide graduate medical education (GME) leaders and executive leaders of hospitals, medical centers, ambulatory care sites, and other clinical settings with formative feedback in the 6 CLER Focus Areas. ${ }^{3}$ This feedback is aimed at improving patient care while optimizing the clinical learning environment (CLE). This report details findings of the first set of CLER Site Visits to 270 ACGMEaccredited Sponsoring Institutions (SIs) with 2 or fewer core residency programs. These site visits to the smaller Sls were conducted from September 9, 2015, to April 18, 2018. The findings from visits to the smallest sites of the Sls visited (ie, preventive medicine programs and single fellowship programs) will be published in a separate report.

The aggregated findings in this report reflect a mixed methods approach (ie, both quantitative and qualitative information gathering and analysis), which was used by the CLER Program to form a comprehensive base of evidence on how the nation's CLEs engage residents and fellows in the CLER Focus Areas.

\section{SELECTION OF CLINICAL LEARNING ENVIRONMENTS}

In 2015, there were 725 ACGME-accredited SIs and nearly 1800 major participating sites, which are the hospitals, medical centers, ambulatory care sites, and other clinical settings where residents and fellows train. This report contains findings from 270 CLEs of the smaller Sls. These CLEs were affiliated with 270 Sls that collectively oversaw 560 residency and fellowship programs (5.4\% of all ACGME programs) and 9818 residents and fellows (7.7\% of all residents and fellows in ACGME-accredited programs). ${ }^{a}$ Appendix A (see p 87) provides additional information on the general characteristics of these Sls (eg, type of SI, number of programs) compared with all ACGME-accredited Sls.

For Sls with 2 or more clinical sites that served as participating sites, the CLER Program visited 1 site due to resource limitations. This selection was based on 2 factors: (1) which CLE served the largest possible number of programs for that SI and (2) whether that CLE had the availability of both the designated institutional official (DIO) and the chief executive officer (CEO) for the opening and exit interviews.

For the majority of the 270 smaller SIs, the CLER Site Visit occurred at the hospital or medical center that served as the major participating clinical site for the SI. At many of these sites, the CLER Site Visitors spent time in both inpatient and affiliated ambulatory care practices. For a small proportion of the Sls, the site visit was conducted exclusively in the ambulatory care setting, including teaching health centers administered by the Health Resources \& Services Administration. Additionally, for a small number of Sls, the site visit was exclusive to an ambulatory care site if the major participating site in an inpatient setting had been visited for a CLER Site Visit for a different SI.

a Source: The ACGME Annual Data Report. The ACGME Annual Data Report contains the most recent data on the programs, institutions, and physicians in graduate medical education as reported by all medical residency Sls and ACGME-accredited programs. 


\section{CLER SITE VISIT PROTOCOL}

In general, the CLER Program designed its site visit protocol to be the same for all CLER Site Visits regardless of the number of core residency programs at an SI. In recognizing that the visits to the smaller Sls involved spending time in both inpatient and affiliated ambulatory care practices or solely in an ambulatory care setting, the CLER Program modified the protocol and site visit process as needed to accommodate these conditions and other unique aspects of the smaller Sls. Overall, these modifications were minor (eg, length of the site visit, site visit agenda) and are noted appropriately in the sections that follow.

The CLER Site Visit protocol included a structured schedule of events for each visit (Figure 1).

\section{Schematic Flow of a CLER Site Visit}

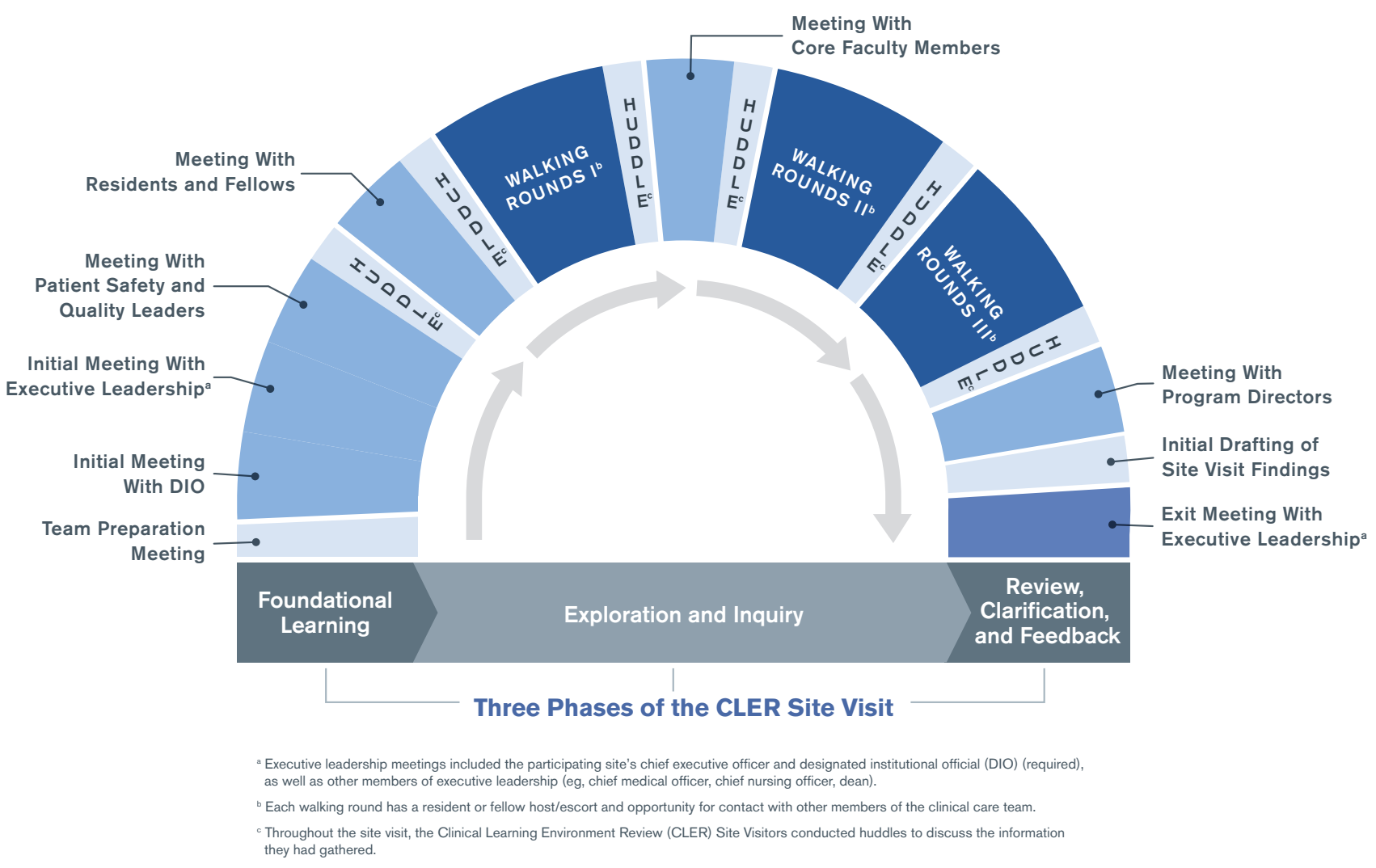

Figure 1. Schematic Flow of a Clinical Learning Environment Review (CLER) Site Visit

CLER Program staff notified clinical sites of their CLER Site Visit at least 10 working days in advance. This relatively short notice was intended to maximize the likelihood of gathering real-time information from interviewees.

All visits to the 270 CLEs of the smaller Sls lasted 2 days. Each CLER Site Visit team comprised 2 CLER Site Visitors (salaried employees of the ACGME). Occasionally, additional team members included other CLER Site Visitors or ACGME staff. 
For site visits that involved time in both inpatient and affiliated ambulatory care practices, the site visit agenda allowed time for the CLER Site Visitors to travel between the inpatient site and the main ambulatory care clinical site.

For the majority of the visits, the CLER Site Visitors conducted group interviews in the same order: (1) an interview with the DIO; (2) an initial group interview with the CEO, members of the executive team (eg, chief medical officer, chief nursing officer), the DIO, and a resident representative; (3) a short interview with patient safety and quality leaders; (4) a group interview with residents and fellows; (5) a group interview with faculty members; (6) a second interview with patient safety and quality leaders ${ }^{\mathrm{b}} ;(7)$ an interview with the program director(s); and (8) an exit meeting with the CEO, members of the executive team, the DIO, and a resident representative. Following specific guidelines, each clinical site provided the CLER Site Visitors with a list of all individuals attending the group interviews before the site visit. The site visitors conducted all group interviews in a quiet location without interruption and ensured that the interviews did not exceed 90 minutes.

The purpose of the initial meetings with executive and patient safety and quality leaders was to allow the CLER Site Visit team to become familiar with the basic language and culture of the CLE's current activities in the 6 CLER Focus Areas. This information helped inform subsequent interviews and observations during the CLER visit.

The resident and fellow group interviews comprised 1 to 18 peer-selected participants per session. Specifically, residents and fellows at the SI, excluding chief residents, voted for their peers to attend the group interviews. The participants broadly represented ACGME-accredited programs at the clinical site with proportionally more individuals from larger programs. The CLER team primarily interviewed residents and fellows who were in their postgraduate year 2 (PGY-2) or higher to ensure that interviewees had sufficient clinical experience to assess the learning environment. PGY-1 residents in a transitional year residency program were permitted to attend.

For the group interviews with faculty members and program directors, the CLER Program instructed the DIO to invite participants to attend the group interviews. In the faculty member group interviews, each session comprised 1 to 23 clinical faculty members who broadly represented the residency and fellowship programs at the CLE. Program directors were not permitted to attend the faculty member meetings. Group interviews with program directors comprised 1 to 8 leaders of ACGME-accredited core residency programs at each clinical site; sessions included associate program directors when program directors were not available.

Additionally, the CLER Site Visit team conducted a set of walking rounds, escorted by senior or chief residents and fellows, to observe various patient floors, units, service areas, and ambulatory care sites. The CLER Program asked the DIO to select residents and fellows, preferably from a range of different specialties, to guide each CLER Site Visitor. For the majority of the smaller SI visits, residents and fellows who participated in the resident and fellow group meetings or who served as the resident representative in the executive leadership meeting were not permitted to serve as escorts for the walking rounds. In CLEs with a small number of residents and fellows, the resident and fellow escorts on the walking rounds were permitted to serve as escorts more than once and could also attend the resident and fellow group meeting.

\footnotetext{
${ }^{\mathrm{b}}$ For the visits conducted exclusively in the ambulatory care setting, the 2 meetings with the patient safety and quality leaders were combined as 1 meeting.
} 
The walking rounds enabled the CLER Site Visit team to gather feedback from physicians, nurses, and other health care professionals (eg, pharmacists, radiology technicians, social workers) in the clinical setting. For the majority of the smaller Sls, each CLER Site Visitor conducted 3 sets of walking rounds per clinical site. For the site visits conducted exclusively in the ambulatory care setting, each CLER Site Visitor conducted 2 sets of walking rounds. All walking rounds lasted 90 minutes.

Throughout each visit, the CLER Site Visit team conducted huddles to discuss the information they had gathered. Later during the visit, they held a team meeting to synthesize their findings, reach consensus, and prepare both an oral report and a draft of a written narrative report. At the exit meeting, the CLER team shared its oral report with executive leadership, which covered initial feedback on the 6 CLER Focus Areas. The written report, delivered approximately 6 to 8 weeks after the site visit, reflected the same topics but with a more comprehensive and detailed set of observations. The intention of both the oral and the written report was to provide formative information that would help executive leadership assess their practices in the 6 CLER Focus Areas, inform resident and fellow training, and guide improvements in the CLE to ensure high-quality patient care.

\section{DATA SOURCES}

\section{Survey Instruments}

To conduct the group interviews, the CLER Site Visitors used a structured questionnaire developed under the guidance of experts in GME and/or the 6 CLER Focus Areas. The questionnaires contained both closed- and open-ended questions. For the site visits exclusive to the ambulatory care setting, the protocol questions and scenarios were modified to fit the setting while keeping the essence of the questions to allow for comparability across settings.

After the questionnaires were initially content validated by expert review, the CLER Program field-tested the instruments on 6 CLER Site Visits-both larger and smaller Sls were included in the field tests. At the conclusion of each of these visits, the items were refined as part of an iterative design process; with each iteration, the CLER Program reviewed and revised the items as necessary based on feedback from interviewees and interviewers.

\section{Walking Rounds}

The CLER Program designed the walking rounds to facilitate random, impromptu interviews with residents, fellows, nurses, and other health care professionals across a number of clinical areas (eg, inpatient areas, emergency departments, ambulatory care settings) where residents and fellows were trained based on the Sl's ACGME-accredited specialty and subspecialty programs.

The aims of the walking rounds were to (1) triangulate, confirm, and cross-check findings from the group interviews and (2) glean new information on residents' and fellows' experiences across the 6 CLER Focus Areas. The walking rounds provided important information that could either confirm or conflict with the information gathered in group interviews.

\section{CLER Site Visit Reports}

The CLER Site Visitors synthesized findings from each visit in a written report, working from a formal template developed and refined in the early stages of the CLER Program. The template assisted the CLER Site Visit 
team in ensuring that each of the 6 CLER Focus Areas was fully addressed in the oral and written reports for each clinical site. The reports also included a brief description of the clinical site and any of its notable aspects. All members of the CLER Site Visit team reviewed and edited each report for accuracy and to achieve consensus on the findings.

In the smaller Sls, the results from group interviews with both the faculty members and the program directors were combined in the oral and written reports to maintain anonymity.

\section{Other Sources of Data}

Several other sources of data were used to augment the site visit data, including the ACGME annual data reports $^{c}$ and the 2015 American Hospital Association (AHA) Annual Survey Database. ${ }^{d}$ The ACGME reports provided information on the Sls, programs, and physicians in GME, including the number of ACGMEaccredited programs, number of residents and fellows matriculated, and university affiliation. The AHA data offered CLE information, including type of ownership (eg, nongovernment, not-for-profit versus investorowned, for-profit) and size, as measured by the number of staffed acute care beds.

\section{DATA COLLECTION}

\section{Group Interviews With an Audience Response System}

CLER Site Visitors conducted group interviews with residents and fellows, faculty members, and program directors using a computerized audience response system (ARS) (Keypoint Interactive version 2.6.6, Innovision Inc, Commerce, MI) that allowed for anonymous answers to closed-ended questions. The ARS data were exported into a Microsoft Excel spreadsheet and then into a software package for statistical analysis. Site visitors documented responses to open-ended questions qualitatively. The 3 surveys -1 each for residents and fellows, faculty members, and program directors-consisted of 45,35 , and 35 closed-ended questions and 25, 24, and 25 open-ended questions, respectively. Of note, 1 question in the resident and fellow survey did not apply to visits exclusively in the ambulatory care setting and thus was omitted from the survey instrument used on these visits.

\section{Group Interviews With No ARS}

CLER Site Visitors documented all responses qualitatively for group interviews with the DIO (35 questions); with the CEO, members of the executive team, the DIO, and the resident representative (34 questions); and with patient safety and quality leadership (70 questions). For the visits exclusively in the ambulatory care setting, 6 questions in the patient safety and quality leadership survey did not apply to this setting and thus were omitted from the survey instrument used on these visits.

\section{DATA ANALYSIS}

\section{Descriptive Statistics}

Descriptive statistics were used to summarize and describe distribution and general characteristics of Sls, CLEs, and physician groups interviewed. For Sls, characteristics included SI type (eg, teaching hospital, medical school) and the number of ACGME-accredited residency and fellowship programs per institution.

\footnotetext{
${ }^{\mathrm{c}}$ The ACGME annual data report contains the most recent data on the programs, institutions, and physicians in GME as reported by all medical residency Sls and ACGME-accredited programs.

d The AHA Annual Survey Database includes data from the AHA Annual Survey of Hospitals, the AHA registration database, the US Census Bureau population data, and information from hospital accrediting bodies and other organizations.
} 
CLE characteristics included type of ownership (eg, nongovernment, not-for-profit), number of licensed beds, and total staff count. Demographic information included gender and medical specialty of physicians who participated in the group interviews.

\section{Analysis of ARS Data}

Analyses were conducted at both the individual (eg, resident and fellow) and the CLE level. For the individuallevel analyses, results are based on the total sample of individuals surveyed, presented as percentages. For CLE-level analyses, results show differences between CLEs after individual responses were aggregated at the CLE level and are presented as medians and interquartile ranges. These 2 levels of analysis provided a national overview of the state of CLE engagement in the 6 CLER Focus Areas and revealed how CLEs compared on these outcomes.

Chi-square analysis was used to compare resident and fellow responses and to identify any relationships in responses by (1) gender; (2) residency year; and (3) specialty grouping. Chi-square analysis was also used to explore if differences were associated with the following CLE characteristics: (1) regional location; (2) bed size; and (3) type of ownership. Categories in the annual AHA survey informed grouping of CLE-specific variables (eg, bed size). $P$ values of .05 or less were considered statistically significant. All statistical analyses were conducted using SPSS Statistics version 22.0 (IBM Corp, Armonk, NY).

\section{Analysis of CLER Site Visit Reports}

Specific findings based on responses to non-ARS questions and interviews on walking rounds were systematically coded in NVivo qualitative data analysis software version 11 (OSR International Pty Ltd, Doncaster, Victoria, Australia) following the principles of content analysis. Three members of the CLER Program staff, trained in qualitative data analysis, generated a master codebook through an iterative process by (1) independently applying codes to the data; (2) peer-reviewing coding; (3) discussing coding discrepancies; and (4) reaching agreement on the codes through consensus. The results were recorded as frequency counts for further descriptive analysis. Overall percentages and percentages stratified by CLE region, bed size, and type of ownership are reported.

\section{Development of Overarching Themes and Findings in the CLER Focus Areas}

Preliminary review of the results revealed that the overarching themes (ie, broad, high-level observations) and the challenges and opportunities (ie, findings) in the CLER Focus Areas were similar to those presented in the CLER National Report of Findings 2018. Due to the similarities, the CLER Program adopted a modified approach to the development of the overarching themes and findings in the CLER Focus Areas for this report.

In the 2018 National Report, the overarching themes and findings in the CLER Focus Areas were determined in 3 stages. First, the CLER Program staff asked each CLER Site Visitor to identify the overarching themes (ie, broad, high-level observations) and the challenges and opportunities in each of the CLER Focus Areas based on their summative experiences and observations through a key informant survey. The CLER Program staff systematically analyzed the content of all responses to discern common themes and note salient concepts. The approach to analysis was inductive in that the themes emerged from the content of the responses.

Next, the CLER Site Visitors reviewed and commented on the results and offered additional findings by consensus. Based on feedback from the site visitors, the CLER Program staff revised the summary of results and presented them to the CLER Evaluation Committee. Lastly, the members of the CLER Evaluation 
Committee reviewed the results and developed a set of commentaries on the importance of the findings and their impact on patient care and physician training. The work of the committee was achieved by consensus.

For this report, the CLER Program staff asked the CLER Site Visitors to confirm or modify the 2018 overarching themes and findings in the CLER Focus Areas based on their overall observations from the site visits to the smaller Sls. The CLER Evaluation Committee then reviewed the results and modified the commentaries as needed.

As part of this modified approach, the CLER Site Visitors also had the opportunity to identify new overarching themes and findings in the CLER Focus Areas, which were developed following the steps described above for the 2018 National Report. Similarly, the CLER Evaluation Committee developed new commentaries by engaging in the same process described above.

\section{Use of Terms to Summarize Quantitative and Qualitative Results}

For the purposes of this report, a specific set of descriptive terms is used to summarize quantitative results from both the ARS and the site visit reports:

$$
\text { few (<10\%), some (10\%-49\%), most (50\%-90\%), and nearly all (>90\%). }
$$

The summary of qualitative data (ie, responses to open-ended questions during group interviews and conversations on walking rounds) is based on the site visitors' assessment of the relative magnitude of responses. The following set of terms is intended to approximate the quantitative terms above:

uncommon or limited, occasionally, many, and generally.

\section{TRIANGULATION AND CROSS-VALIDATION}

Triangulation of the findings enhanced overall accuracy in the conclusions. The findings were cross validated for consistency and corroboration using multiple sources of complementary evidence and analytic techniques. For example, the ARS results were more meaningful when supplemented by critical qualitative information and vice versa. Multiple sources of data provided greater insight and minimized inadequacies of individual data sources when a finding was supported in multiple places. This mixed methods approach provided a richer, more balanced, and comprehensive perspective by allowing for deeper dimensions of the data to emerge.

\section{LIMITATIONS}

As with any formative learning process, limitations to the CLER Program warrant consideration in using the information in this report. Perhaps most important, these findings do not suggest cause and effect.

Second, although this aggregated set of findings is designed to be highly representative, it is based on a series of sampled populations and thus may not be generalizable to all CLEs. As previously mentioned, the CLER Site Visit teams interviewed a sample of residents, fellows, faculty members, program directors, and other clinical and administrative staff for each visit-with the aim of broad representation across all programs (eg, proportionally more individuals from larger programs). Although the goal was to achieve a broad degree 
of representativeness, the sample may or may not reflect the entire population. Given that the CLER Program provides formative assessment, this approach to sampling allowed for a broad and in-depth understanding of socially complex systems such as CLEs. The CLEs that were not included in this sample may represent different experiences and consequently could yield different conclusions as CLER goes on to consider them in the future.

\section{REFERENCES}

1. Weiss KB, Bagian JP, Nasca TJ. The clinical learning environment: the foundation of graduate medical education. JAMA. 2013;309(16):1687-1688.

2. Weiss KB, Wagner R, Nasca TJ. Development, testing, and implementation of the ACGME Clinical Learning Environment Review (CLER) Program. J Grad Med Educ. 2012;4(3):396-398.

3. Weiss KB, Bagian JP, Wagner R. CLER Pathways to Excellence: expectations for an optimal clinical learning environment [executive summary]. J Grad Med Educ. 2014;6(3):610-611.

\section{${ }^{*}$ CLER PROGRAM}

Mark R. Bixby, MD, FAAFP; Isabelle Bourgeois, MPA; Jennifer J. Buescher, MD, MSPH; Robert Casanova, MD, MHPE;

Baretta R. Casey, MD, MPH, FAAFP**; Marian D. Damewood, MD, FACOG; Kevin C. Dellsperger, MD, PhD; Robin Dibner, MD;

Staci A. Fischer, MD; Paula Hensley, MPH; John A. Hopper, MD; Sharhabeel Jwayyed, MD, MS; Catherine Kallal, MD**;

Elizabeth Kimball, MA**; Nancy J. Koh, PhD; Kathryn E. McGoldrick, MD, MAH, FCAl(Hon); Clifton McReynolds, PhD; Terrie Mendelson, MD**; Joshua Mirôn, MA; Robin C. Newton, MD, FACP; Daniel Picard, MD**; Kathy B. Porter, MD, MBA, FACOG;

Dale Ray, MD, MMM; Laura Riordan, MS; Melissa Schori, MD, FACP, MBA; Stephen Smith, MD**; Mike Strickland, MFA*; Hongling Sun, PhD; Marie Trontell, MD; Paul Uhlig, MD, MPA; Robin Wagner, RN, MHSA; Elizabeth Wedemeyer, MD;

Kevin B. Weiss, MD; Esther Woods; Martha S. Wright, MD, MEd; James R. Zaidan, MD, MBA; Jose Zayas, DO, FAAP

**Former staff member 


\section{Overarching Themes}

\section{INTRODUCTION}

As with previous Clinical Learning Environment Review (CLER) national reports ${ }^{1,2}$ this report of the first set of site visits to the smaller Sponsoring Institutions (ie, those with 2 or fewer core residency programs) reveals a number of overarching themes that cut across the CLER Focus Areas. ${ }^{3}$ Of note, the CLER protocol did not directly assess for these themes. Rather, they are based on the CLER Site Visitors' overall observations for this cycle of CLER visits. The development of these themes is described in detail in the Methodology section of this report (pp 15-22).

These overarching themes appear in the following shaded boxes and are numbered for easy reference within the report; these numbers do not suggest order or importance. Each theme is accompanied by a discussion section authored by the CLER Evaluation Committee, which highlights the theme's relevance to the graduate medical education (GME) community and the clinical learning environments (CLEs) in which residents and fellows train.

\section{OVERALL REFLECTIONS OF THE CLER EVALUATION COMMITTEE}

In general, this cycle of CLER Site Visits to the smaller Sponsoring Institutions revealed 4 overarching themes that have carried forward since CLER's 2016 national report $^{1}$ (ie, themes 1 through 4 on the following pages). Theme 6 has carried forward from the 2018 national report. ${ }^{2}$ Theme 5 is a new observation. CLEs face significant challenges in implementing change at the speed and magnitude needed to keep pace with, or ideally anticipate, the future of health care delivery.

The unifying goal for health care systems is to consistently and reliably deliver patient care today that is the safest and highest quality possible. Health care systems that choose to serve as CLEs have the added responsibility of making certain that new learners acquire systems-orientated skills to deliver the highest level of care for the patients of tomorrow.

Transformational change within a CLE requires a complete organizational commitment to quality and safety. Each individual in the organization must model behavior that promotes improvements in patient care. In addition, CLE and GME leadership must collaborate at all levels, from strategic planning, to faculty development, to the front lines of enhancing interprofessional team-based care. When positive relationships and alignment exist, educational and clinical programs are well positioned to demonstrate continued improvements in quality and safety.

Real investment in transformation will likely enhance quality of care and patient care outcomes, as well as create a thriving work climate-improving well-being and retention and yielding overall benefit for the CLE. 


\section{OVERARCHING THEMES}

\section{Theme 1}

Clinical learning environments vary in their approach to and capacity for addressing patient safety and health care quality. In many clinical learning environments, organizational efforts to engage residents in these areas are emerging. Compared with that of residents, there appears to be less focus on participation of fellows in the clinical learning environment's quality and safety activities.

\section{Discussion}

The findings from this first set of CLER Site Visits to the smaller Sponsoring Institutions suggest that CLEs have a number of opportunities in the areas of patient safety and quality improvement that would likely improve the quality of GME as well as patient care.

Acquiring competency in patient safety and quality improvement requires experiential learning. Therefore, engaging residents and fellows in the CLE's quality improvement and patient safety activities is essential. An optimal CLE has consistency of purpose and action with well-articulated strategies, well-defined tools and methods, and common agreement on the role of each member of the clinical team in the organization's patient safety and quality improvement efforts.

To ensure optimal experiential learning, CLEs would benefit from assessing their patient safety and quality improvement activities in the context of how well these programs build competency and capacity for all members of the clinical care team-including residents, fellows, faculty members, and others such as nurses and pharmaciststo create sustainable, system-based solutions for improving care. High-performing CLEs will purposefully design their patient safety and quality improvement programs to engage learners in building competency in these areas.

The findings from the present report suggest that most hospitals, health systems, and ambulatory care sites have operationalized their efforts to address patient safety and health care quality, principally in response to regulatory requirements and performance-based contracting. Success toward these operational objectives can easily co-exist with efforts to create an optimal learning environment that fosters competency of all clinical care team members-including residents and fellows. Optimizing patient safety and health care quality requires systemsbased collaborative team efforts. Therefore, residents and fellows need to be exposed to interprofessional work in patient safety and quality improvement throughout their training. Solutions are more likely to succeed when they are systems based and designed with input from all clinical care team members-including residents and fellows.

The findings from the CLER Site Visits also highlight the need for fellows in subspecialty programs accredited by the Accreditation Council for Graduate Medical Education to be more engaged in quality improvement and patient safety. Some may take the view that fellows should have acquired all of the basic competencies in patient safety and quality improvement while in their core residency programs. However, there are a number of important reasons for fellows to continue to engage in these efforts. First, such engagement will likely benefit patient care. Fellows often care for sicker, more complex patients and need to understand patient safety in this context. Additionally, from an educational perspective, fellows need to learn how to develop and apply patient safety and quality improvement tools and methods within their own subspecialty, which may be very different from the tools and methods used in their previous GME training. Lastly, fellows serve as essential role models for residents of the core residency programs. As role models, they need to develop the knowledge and skills needed to mentor junior colleagues toward professional competency in these areas. 


\section{Theme 2}

Clinical learning environments vary in how they align and collaborate with graduate medical education in developing the organization's strategic goals aimed at improving patient care. In many clinical learning environments, graduate medical education is largely developed and implemented independently of the organization's other areas of strategic planning and focus.

\section{Discussion}

Enhanced collaboration and integration of the CLE and GME can lead to improved patient care. Residents and fellows, who are at the frontlines of patient care, have an excellent knowledge of and ability to manage the patient care experience. These efforts to integrate the CLE and GME can also be viewed as an investment in the organization's clinical workforce.

The findings of the present report suggest that one of the barriers to fully integrating GME into the CLE may be a lack of mutual understanding of how the CLE governance process can help set the strategic direction for optimizing learning in the context of delivering patient care. One example is the absence of stated expectations for GME and other clinical learners in the organization's quality and safety plans.

CLE governance has an important role to play in ensuring that GME is integrated into the CLE's strategic goals for improving patient care. For example, governing bodies can identify how they view GME's contribution to developing the CLE's physician workforce or enhancing the CLE's prestige within their community. In setting the strategic direction for the organization in its role as a CLE, governing bodies can clarify the value of GME within the organization and message the imperative to integrate GME in the development, implementation, and evaluation of strategic goals.

\section{Theme 3}

A limited number of clinical learning environments have designed and implemented educational programs to ensure that all graduate medical education faculty members and program directors have the knowledge, skills, and attitudes necessary for their respective roles in training residents and fellows in patient safety and quality improvement.

\section{Discussion}

To ensure high-quality education for their residents and fellows, CLEs need to ensure that their entire medical staff, particularly faculty members and program directors, is engaged in and able to provide a constructive role in teaching the sciences of patient safety and quality improvement.

Importantly, strong faculty knowledge, skills, and participation in these areas will help CLEs to improve patient safety and health care quality. CLEs that ensure such faculty development will likely see added value by creating a pool of mentors to draw upon year after year. In addition, the CLE will retain some residents after they complete their training, and these junior faculty members will begin their new roles already equipped with these essential skills. 
In addition, faculty development serves a dual purpose-achieving at minimum faculty competency to participate in efforts to improve patient safety and health care quality and ensuring that faculty have the skills and competency to mentor residents and fellows in these areas.

There are both challenges and opportunities associated with implementing an organization-wide plan for faculty development in patient safety and quality improvement. If faculty and staff are to view patient safety and quality improvement activities as an organizational priority, the CLE's executive leadership must message the importance of these efforts, emphasizing the connection to sustainable improvement. They must clearly support such messages with ongoing dedicated resources, successful programs, and accountable goals-all linked to professional advancement.

Executive leadership may also seek to accelerate its plan for faculty development by recruiting individuals with applicable skill sets (eg, patient safety managers, human factors engineers, improvement scientists, implementation coaches) to teach important principles of patient safety and quality improvement and to guide faculty through experiential learning.

Importantly, CLEs that invest in a robust plan for faculty development in patient safety and health care quality are likely to see a reduction in waste, medical liability, and patient harm.

\section{Theme 4}

Clinical learning environments vary in the degree to which they coordinate and implement interprofessional collaborative learning in the context of delivering patient care.

\section{Discussion}

In most CLEs, educational programming appears to focus primarily on acquisition of knowledge and skills specific to each profession. Physicians educate other physicians, nurses educate other nurses, pharmacists educate other pharmacists, etc. The current and evolving practice of medicine necessitates complex, collaborative, team-oriented care and systems-based approaches to coordinating and evaluating health care delivery and outcomes. There are clear needs for interprofessional learning.

Interprofessional education provides a good foundation for learning across the professions based in undergraduate health care education. ${ }^{4}$ There are also models of interprofessional collaborative practice that seek to address this need; however, for many clinicians this type of experience is limited if available at all. Many early learners enter into patient care environments with traditional cultures of siloed professional hierarchy that inhibit collaborative learning and practice..$^{1,2}$

CLEs will excel in providing team-based, collaborative care through developing and implementing programs of interprofessional learning that occurs in the context of the patient care environment.

Highly functioning interprofessional CLEs (IP-CLEs) formally design plans for interprofessional systems-based learning across the clinical workforce-for both early learners such as residents and fellows and learners in other stages of their professional careers. One of the hallmarks of an optimal IP-CLE is a robust collaborative practice model that incorporates structured interprofessional experiential learning as part of routine professional activities. ${ }^{5,6}$ Such a model entails the ongoing attention, support, and oversight of the CLE's executive leaders. 
Ultimately, robust interprofessional collaborative practice, as supported by a high-performing IP-CLE, has the potential to decrease serious patient safety events, increase trust in the clinical care team, improve patient care management and timeliness in care, and improve the efficiency and effectiveness of patient care. Such interprofessional learning can also improve the workforce experience, leading to better recruitment and retention and lower turnover.

\section{Theme 5}

Across clinical learning environments of smaller Sponsoring Institutions, residents' and fellows' experience in health care quality efforts appear to take place within ambulatory care settings. Their experience in health care quality within inpatient settings appears to be less prevalent. In these ambulatory care settings, resident and fellow training also appears to focus more on health care quality than on patient safety.

\section{Discussion}

Understanding health care quality improvement ( $\mathrm{Ol})$ in both ambulatory and inpatient care settings is an essential part of resident and fellow training. As noted in this overarching theme, these clinical learning environments (CLEs) appear to have an enhanced focus on resident and fellow Ql training in ambulatory care settings. This focus is likely leading to residents and fellows with strong ambulatory-oriented QI skills. It also suggests that, in these CLEs, Ol training is less robust in inpatient settings.

Although some residents and fellows may predominantly practice in the ambulatory care setting, they care for patients in both ambulatory and inpatient settings. Therefore, these trainees need experiential learning in $\mathrm{Ql}$ in both environments. Both ambulatory-based and inpatient-based Ql training expose residents and fellows to a number of opportunities and challenges. For example, ambulatory care settings may have longitudinal patient data and a focus on preventive team-based care. They often have patients with chronic illnesses requiring longitudinal complex care. Ol processes in these settings may be managed across different specialty settings and-often-different organizations (eg, consulting with a cardiologist from a different practice). In contrast, inpatient settings may have highly resourced departments; rich data sets from the hospital's electronic health record; and care teams with members all working within the same organization. These settings often have patients with acute care needs, and competition for resources may mean that access to data is limited or in demand by other departments. Additionally, these settings require different health care team configurations and health care resources. Because both ambulatory and inpatient care are important in physicians' clinical activities, it is important to balance residents' and fellows' Ol knowledge, skills, and experience in both of these settings.

The overarching theme also highlights variability in the training that residents and fellows receive in patient safety in the ambulatory environment. This variability is likely a reflection of how patient safety programs are designed, implemented, and deployed in patient care settings outside of the hospital inpatient environment.

The quality of the CLE's patient safety program plays an important role in resident and fellow training in this area. Residents and fellows who train in CLEs with high-quality patient safety programs have a greater opportunity to develop high-quality patient safety skills than those who train in environments without such 
programs. Although ambulatory and inpatient care may have different types of patient safety events, patient safety science principles, methodologies, and expected practice apply regardless of the setting.

Residents and fellows who receive training in $\mathrm{Ql}$ and patient safety that is consistent in both inpatient and outpatient settings will be prepared to address these issues across the continuum of care, ensuring a consistent and high-quality care experience for the patient.

\section{Theme 6}

Health care system consolidation and the concomitant organizational changes in infrastructure, governance, priorities, and values are creating new challenges for clinical learning environments to align graduate medical education with initiatives to improve patient care.

\section{Discussion}

Health care in the United States is undergoing rapid evolution. One of the more notable changes is the degree and pace with which hospitals and other health care environments are consolidating and reorganizing. Often, this consolidation or reorganization reflects a strategic plan of growth through mergers or acquisitions to provide a wider range of health care services to address the movement from hospital-based care to ambulatory, community-based, and home-based services.

Although the reorganization of health care systems is not new, it is now happening at a very rapid pace, which can pose important challenges for GME specifically. Without careful planning, reorganizations place GME educational programming at risk (eg, supervision issues, disruptions to curriculum, ability to meet accreditation requirements); create challenges in the recruitment and retention of faculty and GME leadership; and create challenges in the recruitment of residents and fellows.

Reorganization in US health care also increases risks to patient safety and quality during the transitions due to disruptions and changes in leadership and services. Hospitals and other health care settings that serve as CLEs need to engage GME leadership in any plans for reorganization as early in the process as feasible to mitigate risk of disrupting GME and to ensure patient safety and optimal patient care experiences.

This period of health system reorganization provides an opportunity for Sponsoring Institutions and their respective CLEs to collaboratively define and improve the value that medical education brings to the organization. Furthermore, reorganizations are an ideal time to implement values that optimize the CLE such as collaboration, integration, and shared accountability and oversight-perhaps replacing what was formerly an ad hoc approach to these important principles. At times of reorganization, CLEs have the opportunity raise the bar and apply such principles broadly and consistently at a higher level. 


\section{REFERENCES}

1. Bagian JP, Weiss KB, on behalf of the CLER Evaluation Committee. Overarching themes from the CLER National Report of Findings 2016. J Grad Med Educ. 2016;8(2s1):35-54. doi:10.4300/1949-8349.8.2s1.35

2. Co JPT, Bagian JP, Weiss KB, on behalf of the CLER Evaluation Committee. The overarching themes from the CLER National Report of Findings 2018. J Grad Med Educ. 2018;10(4s):19-24. doi:10.4300/1949-8349.10.4s.49

3. Weiss KB, Bagian JP, Wagner R. CLER Pathways to Excellence: expectations for an optimal clinical learning environment [executive summary]. J Grad Med Educ. 2014;6(3):610-611. doi:10.4300/JGME-D-14-00348.1

4. Cox, Naylor M. Transforming Patient Care: Aligning Interprofessional Education with Clinical Practice Redesign. New York, NY; 2013. doi:10.1023/A:1022480016567

5. Kashner TM, Hettler DL, Zeiss RA, et al. Has interprofessional education changed learning preferences? A national perspective. Health Serv Res. 2017;52(1):268-290. doi:10.1111/1475-6773.12485

6. US Department of Health and Human Services; Health Resources \& Services Administration; National Advisory Council on Nurse Education and Practice. Incorporating interprofessional education and practice into nursing. https://www.hrsa.gov/ advisorycommittees/bhpradvisory/nacnep/Reports/thirteenthreport.pdf. Accessed April 24, 2019.

\section{${ }^{*}$ CLER EVALUATION COMMITTEE}

John Patrick T. Co, MD, MPH, CPPS, FAAP, Co-Chair, Partners Healthcare, Boston, MA; Kevin B. Weiss, MD, Co-Chair, ACGME, Chicago, IL; James P. Bagian, MD, PE**, University of Michigan, Ann Arbor, MI; Terry L. Cline, PhD**, Oklahoma State Department of Health, Oklahoma City, OK; Lindsay Dale, MD, Virginia Commonwealth University, Richmond, VA; David Entwistle, MHSA**, Stanford Health Care, Stanford, CA; Rosemary Gibson, MSc ${ }^{\star *}$, JAMA Internal Medicine, Author, "Wall of Silence," "The Treatment Trap," Arlington, VA; Linda A. Headrick, MD, MS, FACP**, University of Missouri School of Medicine, Columbia, MO; Robert Higgins, MD, Atrium Health, Charlotte, NC; Marcia Hutchinson, MD**, Mercer University School of Medicine, (Savannah Campus), Savannah, GA; LCDR Dinchen Jardine, MD**, Naval Medical Center - Portsmouth, Portsmouth, VA; Lynne M. Kirk, MD, MACP, The University of Texas Southwestern Medical, Center at Dallas, Dallas, TX; Anai N. Kothari, MD, MS**, Loyola University Chicago, Maywood, IL; Catherine M. Kuhn, MD, Duke University Health System, Durham, NC; Tanya Lord, PhD, MPH, Foundation for Healthy Communities, Concord, NH; David Markenson, MD, MBA, FAAP, FACEP, FCCM, New York Medical College, Valhalla, NY; David Mayer, MD, MedStar Institute for Quality \& Safety, Columbia, MD; Douglas E. Paull, MD, MS, FACS, FCCP, CHSE**, VA National Center for Patient Safety, Ann Arbor, MI; Lakshmana Swamy, MD, MBA**, Boston University \& VA Boston Healthcare, Boston, MA; Andrew M. Thomas, MD, MBA**, Wexner Medical Center at The Ohio State University, Columbus, OH; Marjorie S. Wiggins, DNP, MBA, RN, FAAN, NEA-BC, Maine Medical Center, Portland, ME; Ronald Wyatt, MD, MHA, DMS(Hon), Cook County Health and Hospitals, Chicago, IL.

**Past CLER Evaluation Committee member 
30 OVERARCHING THEMES | CLER National Report of Findings 2019 


\section{Challenges and Opportunities in the 6 CLER Focus Areas}

Kevin B. Weiss, MD, and John Patrick T. Co, MD, MPH, CPPS, FAAP, on behalf of the CLER Evaluation Committee*

\section{INTRODUCTION}

The findings in the present report are integral to the nation's understanding of the current condition of how clinical learning environments (CLEs) are engaging residents and fellows in the Clinical Learning Environment Review (CLER) Focus Areas. ${ }^{1}$ They also provide insight on how CLEs can take important steps to purposely enhance the connection between graduate medical education (GME) and optimal patient care. The findings from the first set of CLER Site Visits to the Sponsoring Institutions with 2 or fewer core residency programs suggest that CLEs are raising awareness in the CLER Focus Areas through education and training. These findings also suggest that CLEs are working to establish mechanisms to improve patient safety in their organizations. For example:

- In general, residents and fellows reported awareness of their CLE's process for reporting patient safety events.

- Approximately $86 \%$ of residents and fellows reported knowing their clinical site's priorities in the area of quality improvement.

- Across most CLEs, residents and fellows reported participating in training with nurses and other health care professionals in how to transition patients' care.

- Many CLEs reported efforts to implement online systems by which nurses and other clinical staff members can verify the competency of an individual resident or fellow to perform patient procedures without direct supervision.

- Nearly $90 \%$ of residents and fellows reported that their clinical site provided a supportive, nonpunitive environment for coming forward with concerns regarding honesty in reporting (eg, patient data, duty hours).

As demonstrated in these examples, the present findings revealed positive attributes of the CLEs visited. These findings also revealed continuing challenges and opportunities for improvement. By disseminating these findings, the CLER Program aims to stimulate conversations that result in efforts to improve the quality of the learning environment and patient care.

This section presents the views of the CLER Evaluation Committee on the significance of the findings from the first set of CLER Site Visits to the smaller Sponsoring Institutions and the challenges and opportunities within each of the CLER Focus Areas. The shaded boxes that follow highlight both quantitative and qualitative findings from the CLER Site Visits. (Please refer to the Methodology section on pp 15-22 for more information on the specific terminology used in describing the findings.) As with the section reporting on the overarching themes (pp 24-29), the CLER Program staff presented the CLER Evaluation Committee with a summary of results in each of the CLER Focus Areas to review in the development of this section. The findings are numbered for easy reference within the report. These numbers do not suggest order or importance. 
The following findings highlight the need for CLEs to move from awareness and knowledge to comprehensive training and interprofessional engagement that includes experiential learning and demonstrated competence in the CLER Focus Areas. Additionally, other areas may require CLEs to further explore system-wide strategies to enhance the quality of learning and patient care in clinical settings.

\section{PATIENT SAFETY}

\section{Patient Safety Finding 1}

In general, residents and fellows were aware of their clinical learning environment's (CLE's) process for reporting patient safety events. Some residents and fellows appeared to have used their CLE's patient safety event reporting system.

Residents and fellows appeared to be most comfortable reporting through the chain-of-command and resolving issues at the local or departmental level. Often, these events did not appear to be entered into the CLE's patient safety event reporting system.

When residents or fellows did file a report, or when they had others file it for them, many received little or no feedback from the CLE.

\section{Discussion}

Improving patient safety has to start with those who are closest to daily direct patient care. In this regard, it is essential that residents, fellows, and faculty members fully participate in their CLE's systems and processes for improving patient safety.

The findings from this cycle of CLER Site Visits suggest that GME-based curricular efforts, such as presenting information at resident and fellow orientation and offering web-based modules, are insufficient to ensure effective ongoing engagement in systems-based efforts to address patient safety. To enhance participation, CLEs need to involve residents, fellows, and faculty members in purposeful, planned, and continual experiential learning.

The findings suggest that residents and fellows have an awareness of their CLE's process for reporting patient safety events. However, overall resident, fellow, and faculty reported use of these systems remains modest.

Residents and fellows may not use the CLE's patient safety event reporting system for a variety of reasons, including belief that the chain-of-command is preferred. Resident and fellow behaviors that involve reporting patient safety events through the chain-of-command may be a result of embedded norms. In these situations, GME's hierarchical model of supervision may be unintentionally and counterproductively being applied to patient safety event reporting.

CLEs that primarily rely on the chain-of-command for patient safety event reporting may benefit from examining this approach for its impact on patient care and its effect on role modeling. As reports work their way up the chain from junior residents to senior residents to faculty, important information may be filtered out or lost along the way-thereby preventing the CLE from knowing and sharing the full breadth and extent of the events affecting their patients. CLEs that recognize chain-of-command as a mechanism for patient safety event reporting need to monitor the ability of this approach to ensure optimal input. 
Residents and fellows may also choose not to use the CLE's patient safety event reporting system based on beliefs that reporting is not a physician responsibility and, therefore, can be delegated to other health care team members. This may also be true of faculty members.

It is important that executive and GME leadership use active collaborative interventions to increase faculty engagement in reporting patient safety events, as well as emphasize faculty members' value as role models to residents and fellows. As noted in the 2016 and 2018 CLER national reports, ${ }^{2,3}$ executive leaders have a responsibility to ensure that faculty members have the skills to educate and train residents and fellows to become competent in risk identification, harm reduction, and creating a culture of safety.

Additionally, low levels of reporting among residents, fellows, and faculty members may relate to lack of feedback from the CLE that demonstrates how patient safety event reporting results in improved patient care. When a resident, fellow, or faculty member receives a response to his or her patient safety event report that contains informative feedback with specific improvement efforts, it encourages future reporting. It is important for each patient safety event to be viewed both as a learning experience for the individuals who took the time to report the event, as well as an opportunity to improve patient care.

To optimize reporting, CLEs will likely need a strategy that includes sustained active efforts anchored by demonstrable improvements in patient care that can be directly linked back to the patient safety event reporting system.

\section{Patient Safety Finding 2}

In general, residents, fellows, and nurses lacked clarity and awareness of the range of reportable patient safety events, including what defines a near miss/close call.

When queried, residents, fellows, and nurses also appeared to vary in their understanding of how their clinical learning environment used the reporting of adverse events and near misses/close calls to improve systems of care.

\section{Discussion}

One of the key challenges of patient safety programs is that they focus primarily on harm events. Harm events are much less frequent than nonharm events and occur at the later stages of system failures. Therefore, it is important to emphasize the role of risk-based reporting and to encourage reporting of nonharm events or near misses/close calls.

In the absence of high-functioning systematic approaches to patient safety risk reduction, individuals often use "work-around" efforts to avoid patient safety problems or to expedite patient care. There are numerous reasons why the use of "work-around" efforts to solve system problems is a poor solution for patient care. These 1-time solutions typically solve for an individual's workflow needs and are not system based, which may have unintended consequences. In addition, such solutions offer few shared learning opportunities among team members across units, departments, and service lines-resulting in inconsistent, inefficient, and potentially unsafe care.

CLEs need to ensure that their residents, fellows, faculty, and other members of the health care team clearly recognize what defines a patient safety event or near miss/close call, their responsibility to report it, and the 
value of reports in consistently improving patient care. In addition, it is important that CLEs share the benefits derived from addressing near misses, close calls, and unsafe conditions across clinical units and that all members of the team participate in that learning.

As noted in previous CLER national reports, ${ }^{2,3}$ by broadly communicating the key learnings and improvement plans from patient safety event reviews to residents, fellows, faculty members, and other staff, CLEs can promote the value of systems-based solutions to ensure higher levels of patient safety.

\section{Patient Safety Finding 3}

Across clinical learning environments, a limited number of residents, fellows, and faculty members participated in interprofessional, interdisciplinary, systems-based improvement efforts, such as patient safety event reviews and analyses.

Many residency and fellowship programs used scheduled departmental morbidity and mortality (ie, M\&M) conferences, case conferences, or grand rounds as the primary means of engaging residents and fellows in analyzing patient safety events rather than real-time interprofessional patient safety investigations.

\section{Discussion}

It is likely that the best way for members of the clinical care team to learn to value patient safety event reporting is by participating in interprofessional, interdisciplinary analysis of events. In addition, feedback on patient safety event reports-including individual feedback-encourages reporting and helps all members of the health care team (residents, fellows, faculty members, and others) understand how patient safety can be improved in individual departments and across the organization. As early learners, residents and fellows need a coherent understanding of patient safety event analysis, even though they may experience these analyses in a variety of clinical settings. The variability found across CLEs highlights some key opportunities for improvement. One opportunity is to ensure that, as residents and fellows are exposed to patient safety event analysis, they also understand how the analysis translates into direct value-such as an enhanced culture of safety and actions that lead to rapid improvements in patient care that are monitored for sustained impact.

Many GME communities appear to rely on existing conference structures, such as M\&M conferences or case conferences, as their primary educational activity to ensure residents know how patient safety events are investigated within the CLE. These types of activities are useful and convenient learning opportunities and address the complicated challenge of finding time in resident, fellow, and faculty schedules to learn as a community. They can also provide an educational opportunity for introducing foundational issues in patient safety.

These conference-based educational activities also present substantial challenges to providing residents and fellows with adequate exposure to patient safety event analysis. Conference-based educational activities most often reflect a set of cases that have been prioritized and batched for presentation, resulting in important time delays between the event and the educational activity. Such conferences often place time limits on the discussion of each event because of the need to review multiple events during each session. These group learning opportunities may have special purposes such as regulatory oversight (eg, peer review) or focus on 
issues related to a particularly challenging patient (eg, tumor boards or clinical pathology conferences) and do not necessarily focus on patient safety or address systems-based issues. For many of these conferences, interprofessional participation tends to be infrequent (particularly if the event is also used for peer review).

Considering the challenges, these conference-based activities would best serve as complementary experiences that prepare residents and fellows to participate in real-time, comprehensive patient safety event investigation and analysis.

\section{HEALTH CARE QUALITY}

\section{Health Care Quality Finding 1}

Although most residents and fellows indicated that they participate in quality improvement (QI) projects, many interviewed appeared to have a limited knowledge of $\mathrm{Ol}$ concepts and of the specific methods and approaches to Ql employed by the clinical learning environment.

\section{Discussion}

It has been nearly 20 years since the publication of Crossing the Quality Chasm. ${ }^{4}$ Noting this length of time, it is concerning that the CLER Site Visits have identified variability in residents' and fellows' working knowledge of quality improvement (QI) concepts. During these past decades, hospitals and other health care organizations that serve as CLEs have developed substantial efforts to advance Ql-in part related to regulatory, market, and consumer needs. Yet, it appears that this health system knowledge and experience is not consistently and successfully transferred to residents and fellows during their GME experiences.

As noted in the discussions from previous CLER national reports, ${ }^{2,3}$ didactic educational strategies, while common, appear insufficient. Data from the CLER Site Visits suggest that residents' and fellows' exposure to $\mathrm{Ql}$ is often fragmented. They rarely have the opportunity to work through the full scope of an improvement effort. Instead, they may plan an intervention that they never get to test or implement a change with limited knowledge of the background evidence and no opportunity for follow-up evaluation. Experiential training in all phases of $\mathrm{Ol}$ is necessary for developing the skills essential to improving health care quality. CLEs that do not fully engage residents and fellows in their Ql efforts miss important opportunities to enhance the quality of patient care.

Other sections of this report highlight that GME faculty members also vary in their understanding and practice of QI, and it appears that many CLEs may lack a critical mass of faculty members who are competent in performing and instructing Ql activities. Given the importance of faculty members in delivering and overseeing care and their influence as role models, it is essential for CLEs to invest in faculty development in Ol. For many faculty members, learning about $\mathrm{Ql}$ and mentoring residents and fellows in Ql may have to happen in tandem. 


\section{Health Care Quality Finding 2}

In many clinical learning environments (CLEs), resident and fellow engagement in quality improvement (QI) appeared to be limited to implementing solutions prescribed by the CLE or the resident's or fellow's department. When residents and fellows participated in Ql projects, many of the projects did not align with the CLE's overall goals, were limited in scope, or lacked all of the components of a complete Ql cycle.

A limited number of CLEs integrated OI as part of system-wide efforts to provide residents and fellows with experiential learning aimed at achieving sustained improvements in patient care.

\section{Discussion}

From the residents' and fellows' perspective, learning becomes more meaningful if they can understand the full context of why the CLE is pursuing specific improvement goals and if they can see how efforts in Ol can directly benefit their patients.

As highlighted in the 2016 and 2018 CLER national reports, ${ }^{2,3}$ optimal Ql strategies should include formal, reliable, and routine structural links between the efforts generated by the residents, fellows, and faculty and the CLEs' staff-led efforts to improve care. Coordinating the residents' and fellows' Ql efforts with those of the organization would benefit patients, tap into a rich resource of innovation, and provide the foundation for lifelong $\mathrm{Ql}$ success.

To ensure that residents and fellows are competent in Ql at the end of training, CLEs need to continually expose them to real-world metrics and outcomes that the CLE is tracking to help them understand how the CLE is seeking to improve and achieve its goals.

Although residents and fellows can obtain basic knowledge of $\mathrm{Ql}$ through formal didactic methods or simulated efforts, they acquire lifelong behaviors through real-life experiences and direct engagement in Ql. It is therefore important for this engagement in Ol to begin early in the residents' and fellows' CLE experience and to build throughout their training.

\section{Health Care Quality Finding 3}

In most clinical learning environments, residents and fellows appeared to have limited participation in interprofessional quality improvement teams.

\section{Discussion}

The findings from the CLER Site Visits noted both variability and, often, lack of resident and fellow involvement in interprofessional $\mathrm{Ql}$ efforts. Residents and fellows often view interprofessional engagement as each professional being tasked to implement improvement activities. Genuine interprofessional engagement involves collaboration by all members of the clinical team at each stage of QI work, including planning projects, implementing activities, and monitoring outcomes. 
Substantial challenges exist to achieving full interprofessional engagement in Ql, including schedule demands and rotational assignments. The GME community can overcome these challenges by collaborating with executive leadership across the professions (ie, nursing, pharmacy, social work, and others).

By participating in interprofessional Ql efforts, residents and fellows have the opportunity to gain a comprehensive understanding of how to achieve Ol success through sustainable system-based solutions. Furthermore, interprofessional engagement in Ql provides positive models that enhance interprofessional, collaborative, team-based patient care and workplace well-being.

\section{Health Care Quality Finding 4}

Across clinical learning environments, a limited number of residents and fellows reported access to data on quality metrics and benchmarks for the purposes of quality improvement, including data on outcomes of care for the population of patients for whom they are providing care.

\section{Discussion}

These findings highlight that, often, residents and fellows develop their skills primarily in the context of treating individual patients; they do not develop the skills of using practice-based data (ie, aggregate data on their patient populations) to understand and improve patient care. It is essential that practice-based patient data be part of the formative learning process during residents' and fellows' clinical training. When residents and fellows become independent physicians, the health care systems in which they work will hold them accountable to practice-based data linked to value-based incentive programs and other publically reported data-and, in some cases, these data will be linked to their specialty certification or medical license.

It is important for GME leadership to work closely with executive leadership in both Ql and health information technology (HIT) to improve residents' and fellows' access to and use of practice-based data. Some of the issues to be addressed include determining how the HIT system recognizes the role of residents and fellows and how HIT operations can attribute patient care to residents and fellows. Other issues include identifying the best metrics for assessing resident and fellow learning and patient care, as well as sharing data with residents and fellows that reflect resident and fellow needs and organizational goals and priorities. Data that reflect the interprofessional team are also needed.

CLEs are likely to discover and solve many other important challenges as they work to more closely involve residents and fellows in analyzing and interpreting practice-based data. More importantly, by having residents and fellows spend time with their faculty reflecting on practice-based data, CLEs will promote Ql innovation within their organization. 


\section{Health Care Quality Finding 5}

In a few clinical learning environments, the graduate medical education community has established resident and fellow work groups (such as committees) to increase resident and fellow engagement in quality improvement (QI). Of these work groups, few were integrated with the clinical learning environment's formal Ql processes.

Occasionally, residents and fellows served on departmental Ql committees; fewer were involved in institutional Ql committees. If assigned to these committees, many had limited opportunities for meaningful participation.

\section{Discussion}

Resident and fellow QI work groups are likely to be most effective if they are established to function as part of the formal quality and patient safety operations of the CLE rather than activities based solely in GME. Resident and fellow work groups that are maintained by GME may not be formally integrated into CLE operations. Lack of formal integration is likely to result in inefficient and ineffective communication and misaligned expectations related to Ql innovations, challenges, and successes.

A notable finding is that, occasionally, residents and fellows are being asked to serve on departmental QI committees, and some on organizational Ql committees. To optimize this involvement, GME and CLE leaders must ensure that residents' and fellows' roles are well defined and include the expectation that they participate as full members of the committee. Furthermore, as learners, residents and fellows on Ql committees should be encouraged to ask questions about how the committee does its work and how well the committee is achieving its goals. In addition, they can be a conduit to inform the committee about ongoing GME efforts in patient safety and QI. 


\section{HEALTH CARE DISPARITIES}

\section{Health Care Disparities Finding 1}

Generally, across clinical learning environments, residents and fellows indicated awareness of and were able to describe populations served by the clinical site that were at risk for health care disparities.

\section{Discussion}

The findings suggest that, across many CLEs, residents and fellows are aware of populations at risk for health care disparities. This awareness is an essential early step in building resident and fellow capacity to identify and address health care disparities in their clinical care environments. Part of building such capacity involves recognizing that patients can be at risk for health care disparities through a number of explicit and implicit biases associated with health care delivery.

Optimal CLEs recognize that patients at risk for disparities, as with all patients, require care personalized to their specific needs-based not only on their biological differences, but also on other social determinants of health (eg, personal social support networks, economic factors, cultural factors, safe housing, local food markets)-and impart this knowledge to their residents and fellows.

Disparities in health care that are associated with sociocultural determinants can be a result of CLEs and health care professionals not tailoring treatment according to patients' individual needs or circumstances. Sustainable systems-based solutions ensure that all patients are cared for in a manner that appropriately takes into account the individual's sociocultural background and its impact on health care needs.

Sustainable systems-based solutions also ensure that, early in their GME experience, residents and fellows are able to describe populations who, from the CLE's perspective, are at risk-and understand how these patient populations may receive disparate health care as a result of unintended, unrecognized bias in their individual patient care experiences.

As frontline caregivers, residents and fellows are a valuable resource to the CLE as it formulates strategies to address and eliminate the factors that contribute to health care disparities.

It is also important to note that, as with health care quality and patient safety, residents and fellows cannot learn how to effect positive change around the complex issue of health care disparities unless their faculty and GME leadership have the skills, tools, and data to teach and mentor them in this area. 


\section{Health Care Disparities Finding 2}

Few clinical learning environments appeared to have a formal strategy to address health care disparities or a systematic approach to identify variability in the care or clinical outcomes of their patient populations at risk for health care disparities. A limited number of clinical learning environments were engaged in comprehensive efforts to identify and eliminate health care disparities in a systematic manner; it was uncommon for residents and fellows, faculty members, or program directors to be involved in these efforts.

\section{Discussion}

The CLER Site Visits found that few CLEs have a well-defined or clearly articulated formal strategy to routinely monitor and address health care disparities among the patients to whom they provide care. Therefore, residents and fellows may not learn how best to manage these issues until later in their careers.

In the absence of strategic efforts to address health care disparities within CLEs, residents, fellows, and other health care professionals are likely to develop ad hoc solutions to address individual patient needs. Although such efforts are within health care professionals' direct capacity to achieve, they are not necessarily anchored in sustainable systems-based solutions that benefit other patients with similar risks.

Executive leadership, residents, fellows, and other members of the health care team could serve an important role in developing and implementing robust, systems-based solutions for patient populations with similar special needs-while still allowing for a personalized approach for each patient.

In their efforts to eliminate health care disparities, many CLEs noted they are taking steps to ensure that all patients are treated the same. For example, CLEs may aim to achieve little if any between-patient differences in quality metrics that examine key processes, such as flu vaccination rates or venous thromboembolism prophylaxis.

However, health care disparities can often be missed if addressed solely with this type of outcome assessment that ignores the underlying processes that produce the outcome. For example, studies demonstrate that health care disparities can occur as a result of diagnostic delays or challenges with follow-up or continuity of care. CLEs would benefit from conducting a more systems-based comprehensive assessment of health care disparities among the patients they serve to better understand clinical outcomes that may differ by social, economic, or cultural backgrounds.

Residents and fellows have direct contact with patients and their families. As such, they are in an excellent position to be a critical member of any team working to solve health care disparities. The culture of GME is one of inquiry and learning, and residents and fellows are expected to be engaged in Ql efforts. These efforts could be aligned with those of the CLE toward the common goal of eliminating health care disparities.

Faculty can also be an asset in the CLE's strategic efforts to eliminate health care disparities. To engage faculty, CLEs could align strategic efforts in this area with faculty incentives for best patient care. It is important for CLEs to also provide faculty development to ensure faculty have the skills necessary to create sustainable, systems-based improvements to eliminate health care disparities. 


\section{Health Care Disparities Finding 3}

In addressing health care disparities, many clinical learning environments focused primarily on specific issues such as improving access to care or meeting regulatory requirements. When residents and fellows engaged in addressing health care disparities, it was most often at the level of enhancing patient care access through providing direct service; it was uncommon for them to participate in other systems-based solutions to eliminate health care disparities.

\section{Discussion}

Access to care-limited by financial constraints, workforce shortages, and geographic challenges-is an important contributing factor to health care disparities. At some time during their GME experience, many residents and fellows are likely to participate in efforts to improve patient access to care-such as providing care at community-based clinics for low-income populations or providing care in the context of short-term community outreach projects (eg, health fairs and screening programs).

Access to care, however, is not the only factor that underlies health care disparities. Many non-access-related sociocultural and economic barriers affect patient care as well.

Absent a well-defined and communicated strategy, residents, fellows, and other health care team members learn about populations at risk for health care disparities within their CLE in an ad hoc fashion, often at the point of care. By developing and implementing a strategic plan focused on eliminating health care disparities, CLEs can improve resident and fellow engagement and skill development in this important aspect of health care and thus improve patient care. For the CLE, a strategic plan might include answering some of the following questions:

- What types of health care disparities-beyond issues of access-may unintentionally exist in that CLE's health care system?

- How does the CLE set priorities to address these concerns?

- What are the performance measurements that may address these issues and maintain the success of interventions?

- Can any differences in outcomes be attributed to unequal treatment?

- Conversely, can any differences in outcomes be attributed to failure to consider individualized needswhere certain populations may need more care rather than equal care to achieve the same outcomes?

In addition to the impact on patient care, persistent unaddressed issues in health care disparities can have lasting impact on the health care team, including residents and fellows. Caring for patients at risk for health care disparities can accelerate burnout because health care providers go to extraordinary lengths to meet patient needs absent sustainable, systems-based solutions to support the optimal clinical care of these patients. 


\section{Health Care Disparities Finding 4}

Generally, residents and fellows reported that learning about cultural competency happened informally while providing clinical care. Across most clinical learning environments, formal education and training on cultural competency did not address the specific populations served by the institution.

\section{Discussion}

The diverse, often vulnerable, patient populations served by CLEs provide an important opportunity for teaching residents and fellows to be respectful of patients' cultural differences and beliefs and to recognize the social determinants of health. While, historically, resident and fellow programs have focused on cultural competency, it is now recognized that CLEs' engagement of residents and fellows in the practice of cultural humility is also essential. The practice of cultural humility involves recognizing and reflecting on the possible implicit biases of one culture over another and the limitations of one's capacity to fully understand another person's culture.

In learning and practicing cultural competence and cultural humility, residents and fellows would benefit from moving beyond one-time educational activities (eg, orientation) to a more formal, longitudinal program of progressive educational activities that continues throughout training. Similarly, residents and fellows may benefit from experiential learning within a community context for some of the culturally unique groups in the local environment. These experiences would prepare them not only to address the disparities they face today; they also would contribute to building the skills to manage other unknown challenges in health care disparities that might arise in their future careers.

In optimal CLEs, residents and fellows gain their skills in cultural competency and cultural humility based on experiential learning that involves educating them about how differences in patients' sociocultural backgrounds can affect clinical care, engaging them to identify and implement strategies to address these challenges, and having them apply appropriate assessment tools to determine the efficacy of the actions. CLEs and GME will most likely succeed in this effort via an interprofessional, team-based, systems-oriented approach.

An often-overlooked issue in achieving cultural competency and cultural humility in clinical care is the impact of socioeconomic and cultural differences within the care team, including residents, fellows, faculty, and other clinical staff. It would benefit both patient care and the CLE's workforce engagement if any program designed for improving cultural competency included a focus on understanding these differences among the care team members. 


\section{CARE TRANSITIONS}

\section{Care Transitions Finding 1}

Most clinical learning environments did not appear to have a standardized approach for facilitating resident and fellow change-of-duty handoffs. There appeared to be little understanding of the difference between standardization and uniformity.

In general, residents and fellows lacked awareness and understanding of the importance of standardizing essential elements of the handoff process.

Templates or tools were frequently used to facilitate the handoffs. Across programs and the clinical learning environment, the use of and type of templates varied. It appeared that residents most often engaged in face-to-face handoffs; fellows often conducted handoffs by telephone or e-mail.

\section{Discussion}

In previous CLER national reports, ${ }^{2,3}$ it was noted that, in many CLEs, faculty members and program directors confused standardizing handoffs with a request to create a single and uniform "one-size-fits-all" solution. CLEs and the GME community are encouraged to continue to find solutions that standardize essential properties of the handoff process while allowing for additional specialty- or unit-specific components as needed. Although no generally agreed upon essential properties have been established, several properties are important, including:

- the creation of "to-do" lists;

- the use of "if-then" statements;

- the ability and expectation for the receiver of information to ask questions;

- the practice of "read-back" at the end of a patient handoff; and

- the setting of expectations for when it is essential to move the handoff to the patient's bedside.

It was also noted in previous national reports ${ }^{2,3}$ that patient handoffs are an important communication skill that transcends any individual training program. With their increasing reliance on electronic communication, CLEs would benefit from greater diligence in ensuring that residents and fellows develop the oral communication skills needed to ensure high-fidelity handoffs. Faculty members should supervise and evaluate resident and fellow handoffs in a fashion equal to that of other technical skills. Faculty and senior residents can also be very influential role models for residents and fellows in how patient handoffs should be conducted. By calling attention to the importance of good handoffs, supervision could promote better care transitions throughout the CLE.

A systems-based and standardized approach to patient care handoffs can improve patient safety by ensuring that important elements of care are communicated in an efficient and reliable manner. Ensuring that residents and fellows have the skills to conduct effective and reliable patient handoffs increases the quality of transitions, decreases errors of omission, eliminates confusion of the handoff process or the information that 
is being relayed, and enhances professionalism. Standardization also may decrease anxiety by providing those on the receiving end of the transfer with a clear narrative of patient care expectations. Having a standardized approach to handoffs also allows faculty members to provide a more consistent assessment of resident and fellow skills in transitioning care.

CLEs would benefit from establishing a common set of expectations of what they-as a CLE-would define as an appropriate handoff. With a common set of expectations, all of the health care professionals in the CLE could align clinical care goals.

\section{Care Transitions Finding 2}

Residents, fellows, and nurses expressed concerns that communication during transitions of care was often incomplete or inaccurate and created risk to patient safety. Examples included transitions from the emergency department to inpatient care, from service to service in inpatient settings, from inpatient care to outpatient care, and from one hospital to another, as well as transitions involving skilled nursing facilities or rehabilitation settings.

A standardized, organization-wide approach to training in and managing transitions in care between clinical services assigned to resident and fellow teams (eg, emergency department to inpatient care, operating room to intensive care unit, intensive care unit to floor, and medicine to surgery) was uncommon across clinical learning environments.

\section{Discussion}

There are many advantages to including residents and fellows in strategic planning around transitions of care. As individuals at the frontlines of care, residents and fellows often see firsthand the consequences of ineffective and inefficient care transitions. When residents' and fellows' role in such strategies is limited to implementing changes designed by others, they lose the opportunity to gain experience in developing systemsbased approaches to Ql.

Similarly, when executive leadership excludes residents and fellows from their QI activities around transitions of care, they run the risk of creating suboptimal solutions, such as preventable readmissions. Nearly all transitions of patient care necessitate a team effort that, in addition to the patient, includes clinical staff, administrative support, and CLE operations. Residents and fellows can bring unique insights to improving these critical junctures of patient care. 


\section{Care Transitions Finding 3}

Across clinical learning environments, a limited number of programs appeared to use formal criteria to assess residents' and fellows' skills in change-of-duty handoffs. Across programs, it was uncommon to find faculty members consistently engaged in direct observation of resident and fellow change-of-duty handoffs. When faculty members were involved, the level of engagement and the process for supervision varied. Little or no monitoring of change-of-duty handoffs by graduate medical education leadership, executive leadership, or patient safety and quality leaders of the clinical learning environment was reported.

\section{Discussion}

Change-of-duty handoffs are high-risk moments in patient care and, as such, necessitate skill building for optimal performance. Some of these skills can be developed through validated simulation-based education strategies. However, it is important that much of this skill development occur during supervised clinical care.

In developing their skills, residents and fellows benefit from observing and participating in handoffs that include clinicians with many years of experience in both simulated and authentic patient care experiences. When handoffs are conducted solely among residents who are very early in training, the residents do not have the opportunity to learn from the expertise and advanced skills of more senior residents, fellows, or faculty.

Assessment and monitoring of handoffs involves skill building for the faculty as well and is an important component of faculty development. Achieving high-fidelity handoffs requires continual faculty diligence in role modeling, observing, assessing, monitoring, and providing constructive feedback to learners on their performance.

CLEs with faculty who are vigilant in their attention to building residents' and fellows' skills in handoffs and transitions of care contribute to patient safety and improvement in patient care. 


\section{SUPERVISION}

\section{Supervision Finding 1}

Across most clinical learning environments, residents, fellows, and faculty members reported an overall culture of adequate supervision within the graduate medical education community.

Clinical learning environments also faced challenges of under- and oversupervision.

- Residents, fellows, faculty members, and program directors perceived that undersupervision occurred mainly during times of high acuity, high patient volume, nights and weekends when the number of faculty members available to supervise was limited, and when the demands of competing clinical responsibilities exceeded the capacity of faculty members to provide adequate supervision.

- Faculty members and program directors perceived that external factors were contributing to oversupervision that impeded resident and fellow readiness for clinical practice after training. The most common reasons given for concerns regarding oversupervision related to billing rules and medical liability concerns.

\section{Discussion}

As seen in previous CLER national reports, ${ }^{2,3}$ the findings illustrate the inherent challenge that CLEs have in achieving the right balance in supervision, an issue that is necessarily complicated by the need for different levels of supervision at different levels of training.

Oversupervision of residents and fellows can have the negative consequence of producing physicians who are unprepared for independent practice. This lack of preparedness may contribute to patient safety risks and, for the physicians, may contribute to stress and burnout. Also, as noted in the 2016 and 2018 national reports, ${ }^{2,3}$ billing requirements, payment policies, and regulatory and accreditation rules appear to continue to influence CLEs and residency programs by placing significant restrictions on the amount of patient care that residents and fellows can perform without close direct supervision. An environment in which residents/fellows are oversupervised can lead to a cycle that is demoralizing to residents and fellows, decreasing their ownership of patient care and thus perpetuating oversupervision due to faculty concerns of their competence. The privilege of progressive autonomy, on the other hand, promotes a learning environment in which residents/fellows can build confidence and grow in their clinical competence.

Alternatively, undersupervision potentially creates patient safety vulnerabilities, as well as contributes to residents' and fellows' stress, anxiety, and possible burnout-all factors affecting patient safety as well. Undersupervision also decreases the opportunities for teaching and learning.

The issues of undersupervision and oversupervision are necessarily complex and demand active, constant engagement and monitoring through a collaborative effort between GME leadership and the CLE's executive leadership. 


\section{Supervision Finding 2}

Across many clinical learning environments, residents and fellows expressed concerns about their peers providing consultative services without adequate supervision, leading to patient safety vulnerabilities.

\section{Discussion}

This finding highlights the importance of adequate supervision of resident and fellow consultative services within a teaching environment. Consultations are a major source of learning for residents and fellows, and undersupervised consultations can be important missed learning opportunities. They can also become preconditions to patient safety events. Although the concerns detailed in this finding were specific to peerto-peer consultation, they may also be a reflection of the larger clinical learning environment and consultative processes in general.

Consultative services form a critical component of most patient care. Consultations can be inefficient due to delays in completing the consultation, perceived lack of responsiveness to the reason for the consultation, and inadequate communication of findings and recommendations, all of which can adversely affect patient care.

Many factors potentially contribute to inefficient and ineffective consultations. The referring resident or fellow may create delays in initiating a consultation or not state clearly the reason for the consultation. Both of these problems can be mitigated by appropriate supervision and mentorship. Similarly, the consulting physician may not complete the consultation in a sufficiently timely fashion to optimally address patient care needs or may fail to directly address the reason for the referral. These challenges are more likely to arise when consultative services are unsupervised and are of particular concern during nights and weekends, when direct communication with a faculty consultant may be more difficult to secure.

To ensure that patients receive expert and high-quality care throughout the care process, executive leadership may want to consider periodically assessing the quality of consultative services as part of their systems approach to improving patient safety and health care quality.

\section{Supervision Finding 3}

Across many clinical learning environments, residents and fellows expressed reluctance to request help from the attending physician, noncore faculty, and consultants or to report concerns regarding supervision. Residents and fellows were hesitant to ask for assistance for several reasons, including a lack of understanding about when to escalate concerns to a supervisor; an unwillingness to appear unprepared by asking for assistance; a fear of retaliation; a sense of shame; and concerns of pushback from peers, attending physicians, and consultants.

\section{Discussion}

A culture of patient safety and learning best operates in an environment that is psychologically safe for the residents and fellows. Faculty and consultant behaviors that instill residents and fellows with a sense of shame or fear of inquiry adversely affect learning and worsen the quality of patient care. Therefore, it may be helpful for 
CLEs to ensure that they train residents, fellows, and faculty on expectations related to seeking or responding to requests for assistance.

Senior residents, fellows, faculty members, and consultants need to have supervisory systems that eliminate barriers to requests for assistance. Senior residents, fellows, faculty members, and consultants need to be supportive regardless of the quality of the inquiry-no matter the time of day or day of the week.

Executive leadership in collaboration with the GME leadership needs to develop and implement mechanisms that optimize skills in supervision and find ways to recognize and value faculty members and consultants who model desired supervisory behaviors.

\section{Supervision Finding 4}

Many clinical learning environments made efforts to implement online systems by which nurses and other clinical staff members could verify the competency of an individual resident or fellow to perform various patient procedures without direct supervision. When an online system was available, nurses were not aware of its existence, did not know how to access it, or rarely used it.

Across many clinical learning environments, nurses indicated that, in the absence of an attending physician, they relied on familiarity, trust, or year of training.

\section{Discussion}

Patient safety and quality departments could benefit from working with GME programs to jointly develop proactive monitoring of residents and fellows. For example, if the CLE provided accessible and transparent information regarding which procedures an individual resident or fellow is competent to perform, other members of the care team could more easily determine when direct supervision is needed. However, these systems are likely to be underused unless the CLE clearly communicates expectations regarding use of this information in the daily workflow of clinical care. Many CLEs may need educational activities to assist the nursing staff in understanding their important roles and responsibilities in working with the GME faculty to ensure optimal supervision and safe patient care.

It appears that patient care may benefit from CLEs strengthening their systems for interprofessional involvement that ensure that residents and fellows are always practicing under the most appropriate level of supervision. It is important that such systems are a standard part of the workflow process rather than based on individual judgment. Behaviors that are established as a routine part of the workflow process decrease defensiveness and minimize the potential for expected actions to be misinterpreted. 


\section{Supervision Finding 5}

Residents and fellows, faculty members, program directors, graduate medical education leadership, patient safety leadership, and executive leadership varied in their awareness of patient safety events related to supervision.

In general, the executive leadership and the patient safety and quality leaders of the clinical learning environments indicated that they did not actively monitor supervision of residents and fellows. They indicated that monitoring is limited to retrospective review of patient safety events. Responsibility for resident and fellow supervision was viewed as primarily the purview of the graduate medical education community. Across clinical learning environments, some program directors reported having managed issues related to resident and fellow supervision within the past year that resulted in a patient safety event.

\section{Discussion}

Comprehensive solutions for adequate, but not excessive, supervision of resident and fellow physicians within CLEs entail ongoing attention and monitoring. Although this responsibility is centered within GME, it is essential for patient care that it include regular review by the executive leadership of CLEs.

Involvement of CLE executive leadership on issues of supervision can lead to a more comprehensive awareness of the clinical areas where risks to patient safety exist and where improvements to patient care are needed. The GME community is responsible for the foundational management of resident supervision. However, the patient care environment will benefit only if both GME and executive leadership of the CLE create a clear, aligned vision of supervision requirements and monitor it closely. 


\section{FATIGUE MANAGEMENT, MITIGATION, AND DUTY HOURS}

\section{Fatigue Management, Mitigation, and Duty Hours Finding 1}

When provided with a scenario of being maximally fatigued 2 hours before sign-off, across clinical learning environments, some residents and fellows reported that they would continue to work until their sign-off rather than expect to be taken off duty. When presented with the same scenario, faculty members and program directors were less likely to express the belief that residents and fellows would continue to work under such circumstances.

\section{Discussion}

The CLER Site Visit protocol for this report of findings was largely conducted before the 2017 release of the updated Section VI of the ACGME Common Program Requirements ${ }^{5}$-and its emphasis on fatigue management and mitigation. The present findings primarily reflect the CLEs' experiences under the 2011 Common Program Requirements Section VI. ${ }^{6}$

The revisions to Section VI of the Common Program Requirements released in 2017 have relaxed the requirement for the 16-hour workday for a postgraduate year 1 resident. ${ }^{7}$ In this transition of Common Program Requirements, it is essential for patient safety that CLEs develop and maintain close monitoring of and support for residents and fellows who are fatigued while caring for their patients.

Respective of the changes in the Common Program Requirements, the findings suggest that, in some CLEs, residents and fellows view fatigue management interventions as optional. The responses to the scenario suggest that residents and fellows do not necessarily appreciate how fatigue management links to patient safety and clinical outcomes. It is the CLE's culture of patient safety that establishes the expectations for resident and fellow behavior around fatigue management.

It is recognized that faculty and residents and fellows who are in their senior years of training may face exceptional situations when they need to weigh the balances of working while fatigued versus transitioning care. When CLEs have experiential learning, assessment, and feedback to proactively address fatigue management, residents and fellows learn how to make the best judgments related to their personal fatigue and patient safety, skills that they can draw on throughout their professional career. Strong fatigue management systems enable CLEs to ensure the highest level of patient care while being conscious about stress and fatigue among its residents and fellows.

CLEs that have robust systems for fatigue management and mitigation should expect residents and fellows to consistently use them. When fatigue mitigation strategies are not used in a consistent manner, the CLE is at increased risk of adverse impact on patient care. 


\section{Fatigue Management, Mitigation, and Duty Hours Finding 2}

In many clinical learning environments, residents and fellows described witnessing signs of burnout in a number of their colleagues. The main contributors to resident and fellow burnout related to high patient volume, patient acuity, and nonphysician responsibilities. Also, residents and fellows reported observing signs of burnout among faculty members and program directors.

Faculty members and program directors reported the same contributing factors identified by residents and fellows and emphasized clinical productivity pressures, extensive documentation requirements, inadequate clinical and administrative support, and the overall challenge of balancing teaching, research, administrative responsibilities, and patient care.

\section{Discussion}

As noted in the finding above, in many CLEs, residents, fellows, faculty, and program directors witnessed signs of burnout. These findings are an important signal to both the GME community and CLEs, as they reveal a problem with the physician workforce that has serious potential for adversely affecting patient care.

For the CLE, physician burnout can lead to loss of empathy for patients and frustration on the part of patients and family members. In addition, burnout degrades the ability to learn and teach and contributes to lower quality performance and poor morale in the workplace, which can negatively affect the entire care team. Physician burnout can also lead to unprofessional behavior and risk for self-harm. All of these factors can increase medical liability, workforce turnover, institutional costs, and risk of suboptimal patient outcomes.

Residents and fellows often incorporate the skills and behaviors modeled by faculty into their own current and future clinical care. Seeing burnout among their faculty members is likely to influence how they manage their future careers and perceive their future career choices.

The issue of physician burnout is extremely complex and has become the subject of not only the medical profession but, more broadly, of the health care workforce in general. ${ }^{7}$ In optimal CLEs, faculty members are involved in both the design and the implementation of strategies to prevent burnout.

A number of factors related to financial productivity, patient complexity, and regulatory requirements have increased faculty workload over the past decade. The ACGME, through accreditation requirements and attention to the maximum number of duty hours per week, has encouraged better fatigue management for residents and fellows. However, no comparable widespread guidelines exist for faculty, resulting in increased fatigue, patient safety vulnerability, burnout, and insufficient role modeling of behaviors to manage and mitigate fatigue.

Ultimately, patient care will benefit from CLEs taking systematic approaches to burnout management and prevention that include all members of the patient care team. These approaches would best benefit the CLE if they were anchored in the strategic goals and measured as part of the organization's performance management activities. 


\section{Fatigue Management, Mitigation, and Duty Hours Finding 3}

In general, clinical learning environments had developed and implemented some form of fatigue management for residents and fellows. Mitigation focused mainly on provision of sleeping facilities (eg, designated call rooms) and transportation options (eg, taxi services).

A limited number of clinical learning environments had systematic strategies and solutions that focused on prevention, recognition, and effective mitigation of fatigue and burnout. If strategies existed, they were generally in response to an event related to fatigue or burnout.

\section{Discussion}

As previously mentioned, the CLER protocol for this report of findings was conducted primarily before the release of the updated Section VI of the ACGME Common Program Requirements in 2017-and its emphasis on fatigue management and mitigation. ${ }^{5}$ The findings primarily reflect the CLE experiences under the prior version of the Common Program Requirements Section VI. ${ }^{6}$

It appears that, while CLEs met their responsibilities to implement basic strategies for fatigue management, residents, fellows, faculty members, and nurses still reported instances of resident and fellow fatigue. Fatigued health care providers can place patients at risk for medical errors and also harm their own health and others (eg, car accidents, needle sticks, burnout). Fatigue management is about both patient safety and health care provider well-being and safety.

At the time of these CLER Site Visits, across most CLEs, assessment of resident and fellow fatigue appeared to be largely limited to monitoring the number of hours worked. Yet, many other factors can cause fatigue, including task or mental overload due to high-volume or high-acuity patient activity, circadian rhythm disruption, chronic sleep deficit, and non-work-related activities. Moreover, "fatigue" can be a risk factor for burnout or depression. ${ }^{8}$ Optimal CLEs train residents, fellows, faculty members, and other clinical staff to consider such factors-and not only duty hours-in determining a health care provider's "fitness for duty."

CLEs may benefit from implementing more advanced organizational strategies for fatigue management, such as scheduling the clinical workforce to maximize rest and account for circadian rhythms, allowing for strategic naps, batching calls to residents and fellows where appropriate, and implementing systems to relieve tired health care providers from direct patient care responsibilities. Strategies to address system and work process issues contributing to fatigue need to be informed by the interprofessional frontline care delivery experience in development and implementation. This approach to thinking about fatigue management and mitigation is embedded in the philosophy of the ACGME's 2017 Common Program Requirements, ${ }^{5}$ providing a GME lens for effecting change.

For meaningful change to occur, CLEs need to progress from individual tactics toward system-wide strategies that are implemented and routinely monitored to ensure their efficacy. Ultimately, rapid evolution to address the issue of fatigue management will necessitate the GME community working with their CLEs to effect broader system change that is directly linked to safe patient care. 


\section{PROFESSIONALISM}

\section{Professionalism Finding 1}

In many clinical learning environments, graduate medical education and executive leadership expressed intolerance for behaviors that are considered unprofessional. Across some clinical learning environments, residents, fellows, and clinical staff described witnessing or experiencing incidents of disrespectful or disruptive behavior on the part of attending physicians, residents, fellows, nurses, or other clinical staff. These findings ranged from descriptions of isolated incidents to reports of disrespectful behavior that was persistent or chronic in nature.

\section{Discussion}

Although most CLEs appear to have mechanisms to address disruptive and disrespectful behaviors of their trainees, faculty, and other clinical staff, the findings suggest that existing mechanisms are neither consistently applied nor effective. It appears that many organizations may be equating a culture of no tolerance for unprofessional behavior with acceptance of low prevalence of these behaviors, resulting in normalization of deviant behaviors.

Isolated episodes of unprofessional behavior are often different from chronic, persistent unprofessional behavior. Any chronic, persistent unprofessional behavior-either directed toward residents and fellows or emanating from residents and fellows toward other clinical team members-reflects negatively on the CLE and GME leadership and suggests their lack of ability to adequately address the issues. An optimal organizational culture includes effective ways of quickly and successfully identifying, remediating, and preventing these behaviors.

Efforts to deal with unprofessional behavior need to ensure the consistent application of standards, avoiding capricious exceptions. In addition, CLEs need to enhance their efforts to seek out and address issues of professionalism that could be detrimental to patient safety, impede quality improvement efforts, or foster an unacceptable culture within the GME community and among hospital/medical center staff.

The persistent search for and elimination of unprofessional behavior over time creates confidence in the clinical team to conduct its work in a psychologically safe environment that positively and directly impacts patient care.

\section{Professionalism Finding 2}

Residents and fellows reported instances of feeling pressured to compromise their integrity to satisfy an authority figure.

\section{Discussion}

The finding presents an opportunity to better understand how the influence of senior residents, faculty, and GME leaders shapes the professional identity of physicians in their early stages of postgraduate medical education. 
Many reasons exist for why a resident or fellow may feel pressured to compromise his or her integrity. Examples vary widely and include lack of knowledge on a subject-creating a false or imperfect assessment of the situation-and dishonest behavior on the part of a supervisor. In either example, it is essential that residents and fellows are comfortable and safe in raising concerns and are not placed in situations where they feel coerced into being dishonest.

In developing their professional identity, residents and fellows progressively construct a framework for viewing professional integrity in the context of delivering patient care. This framework is developed dynamically based on continual feedback from colleagues and mentors and is likely influenced by other members of the CLE in leadership roles as well.

Supporting residents and fellows as they develop their professional identity is primarily the responsibility of the GME community and is guided-in part-through assessment of competency. However, it is the responsibility of CLE executive leadership to establish and maintain a culture where residents and fellows (as well as other staff) can raise concerns without worries or fears of retribution.

The ultimate responsibility for creating and maintaining a safe culture rests with the CLE, as it directly affects the quality of patient care. In this regard, executive leadership has an important responsibility to ensure a clinical care environment where residents and fellows are invited and expected to speak up about issues of professional concern and are supported in the process.

\section{Professionalism Finding 3}

Across clinical learning environments, residents and fellows described experiencing professionalism issues in obtaining consultation services (eg, delays or lack of responsiveness to providing assistance in patient care, disrespectful communication in response to requests).

\section{Discussion}

All physicians need to call upon the expertise of other colleagues to help them make better diagnostic and treatment decisions. Unprofessional behavior on the part of consultative physicians is a detriment to safe and high-quality patient care. An unprofessional consultative experience may yield many untoward consequences, such as increased likelihood of errors in decision making due to incomplete communication, delays in diagnosis and treatment, insufficient supervision, antagonistic relationships between clinical services, and worst of all, patient harm.

Findings of unprofessional behavior related to consultative services are, to a large degree, a reflection of the CLE's culture and therefore a reflection of its executive leadership and attentiveness to the effects of culture on patient care.

A CLE can improve the quality of consultations in many ways, including: clarifying the value of consultations and the importance of asking for help, focusing on the value of teamwork, fostering respectful interdisciplinary interactions, and monitoring and intervening in response to signals of unprofessional behavior. They can also offer technology and other tools such as secure text messaging to facilitate rapid communications.

By ensuring consistent, high-quality consultative services at all times, CLEs can improve both the patient care experience and resident and fellow learning. 


\section{Professionalism Finding 4}

Generally, residents and fellows appeared to be aware of the mechanisms and resources available to resolve perceived mistreatment if seeking assistance beyond those offered by the graduate medical education program. The perceived effectiveness of the institution's response varied across CLEs.

Occasionally, residents and fellows indicated that they would not report mistreatment out of concern for adverse consequences of reporting.

\section{Discussion}

Optimal CLEs establish clear mechanisms both within and outside of GME leadership for residents and fellows to follow in reporting and addressing unprofessional behavior. Ideally, these various mechanisms feed into a single repository so that leadership can identify themes, frequency, and chronicity of concerns about professionalism and potentially share lessons learned across the organization.

Residents or fellows may not report unprofessional behavior that they have experienced or witnessed if they feel as though they do not have anyone to report to outside of GME or if they are confused over whom to report to. Alternatively, in such a situation, they may go outside the organization without exhausting opportunities to address their concerns within the CLE and create sustainable solutions that may mitigate the situation for other learners.

Ensuring that residents and fellows (and other health care professionals) have a high degree of psychological safety can only benefit patient care and optimize learning. Enhancing collaboration between the CLE and GME leadership in tracking and managing reports of unprofessional behavior will promote learning that benefits both the CLE and its GME programs.

Many GME leaders may believe that their residents and fellows operate in an open, safe, protective environment with no need to seek assistance outside of their GME community. However, multiple reasons exist for why a resident or fellow may feel unsafe, and these may or may not be related to the overall quality of GME. Therefore, for the well-being of the residents and fellows, it is essential that they have a well-supported, easily accessible, known, and trusted non-GME pathway for seeking assistance when faced with unprofessional behavior.

Having psychological safety at work is one of the fundamental mainstays for patient safety and health care quality. It is the responsibility of the CLE's leadership to ensure that they have such mechanisms to support well-being not only for residents and fellows but also for all of the members of the health care team. 


\section{Professionalism Finding 5}

Across clinical learning environments, some residents and fellows reported documenting history and physical findings in a patient's health record that they did not personally elicit (such as copying and pasting in the electronic health record without proper attribution).

\section{Discussion}

The deployment of electronic health records (EHRs) across the United States over the past decade has led to myriad challenges. The finding that some residents and fellows reported documenting history and physical findings in a patient's health record that they did not personally elicit is concerning and may be the result of CLEs distributing technology without supporting its health care professionals in developing the skills needed to manage change from a paper-based health record system to an EHR system. Many EHRs have functions that prepopulate areas of the patient's medical record, and for some EHRs, it is relatively easy to copy a note from a prior section of the patient's history or physical examination findings and place it in another note. These workflows by themselves are not the focus of this finding.

Residents and fellows may have many compelling reasons to copy and paste work from others. Primary among such reasons is that this practice may decrease the amount of unnecessary duplication of work-an important factor in a health care environment that focuses on patient volume and clinical productivity.

It is the lack of proper attribution that raises concern. Copying without appropriate attribution results in documentation that represents the efforts of some other individual. Most importantly, this activity can lead to perpetuation of inaccuracies in documentation, thereby increasing the risk of patient harm. Copying and pasting without attribution can also lead to increased medical liability exposure.

Copying and pasting is a workaround to a system problem and a reflection of a system impediment. It is the CLE's responsibility to address this impediment and implement an EHR that minimizes the need for copying and pasting and maximizes correct attribution when the function is used appropriately (eg, bringing forward laboratory data or vaccination dates).

Solving this issue involves close collaboration between the CLE's executive leadership, the department of health information technology, and the health care professionals (including residents and fellows) who are on the frontlines of patient care.

Work in this area is likely to be ongoing for some time, as this challenge for CLEs is also a challenge for the health care industry at large. It is important that these challenges are viewed as high priority, as they affect patient safety and the quality of care. 


\section{REFERENCES}

1. Weiss KB, Bagian JP, Wagner R. CLER Pathways to Excellence: expectations for an optimal clinical learning environment [executive summary]. J Grad Med Educ. 2014;6(3):610-611. doi:10.4300/JGME-D-14-00348.1

2. Weiss KB, Bagian JP, on behalf of the CLER Evaluation Committee. Challenges and opportunities in the six focus areas: CLER National Report of Findings 2016. J Grad Med Educ. 2016;(2s1):25-34.

3. Weiss KB, Co JPT, Bagian JP, on behalf of the CLER Evaluation Committee. Challenges and opportunities in the 6 focus areas: CLER National Report of Findings 2018. J Grad Med Educ. 2018;10(4s):25-48. doi:10.4300/1949-8349.8.2s1.25

4. Committee on Quality of Health Care in America; Institute of Medicine. Crossing the Quality Chasm: A New Health System for the 21st Century. Washington, DC; 2001.

5. Accreditation Council for Graduate Medical Education. Common Program Requirements. Chicago, IL; 2017. https://www. acgme.org/Portals/O/PFAssets/ProgramRequirements/CPRs_2017-07-01.pdf.

6. Accreditation Council for Graduate Medical Education. Summary of changes to ACGME common program requirements section Vl. http://www.acgme.org/What-We-Do/Accreditation/Common-Program-Requirements/Summary-of-ProposedChanges-to-ACGME-Common-Program-Requirements-Section-VI. Published 2017.

7. Dzau VJ, Kirch DG, Nasca TJ. To care is human-collectively confronting the clinician-burnout crisis. N Engl J Med. 2018;378(4):312-314.

8. Dyrbye LN, Shanafelt TD, Sinsky C. Burnout Among Health Care Professionals: A Call to Explore and Address This Underrecognized Threat to Safe, High-Quality Care. Washington, DC; 2017. https://nam.edu/burnout-among-health-care professionals-a-call-to-explore-and-address-this-underrecognized-threat-to-safehigh-quality-care.

\section{${ }^{*}$ CLER EVALUATION COMMITTEE}

John Patrick T. Co, MD, MPH, CPPS, FAAP, Co-Chair, Partners Healthcare, Boston, MA; Kevin B. Weiss, MD, Co-Chair, ACGME, Chicago, IL; James P. Bagian, MD, PE**, University of Michigan, Ann Arbor, MI; Terry L. Cline, PhD**, Oklahoma State Department of Health, Oklahoma City, OK; Lindsay Dale, MD, Virginia Commonwealth University, Richmond, VA; David Entwistle, MHSA ${ }^{\star \star}$, Stanford Health Care, Stanford, CA; Rosemary Gibson, MSc ${ }^{\star \star}, J A M A$ Internal Medicine, Author, "Wall of Silence," "The Treatment Trap," Arlington, VA; Linda A. Headrick, MD, MS, FACP**, University of Missouri School of Medicine, Columbia, MO; Robert Higgins, MD, Atrium Health, Charlotte, NC; Marcia Hutchinson, MD**, Mercer University School of Medicine, (Savannah Campus), Savannah, GA; LCDR Dinchen Jardine, MD**, Naval Medical Center - Portsmouth, Portsmouth, VA; Lynne M. Kirk, MD, MACP, The University of Texas Southwestern Medical, Center at Dallas, Dallas, TX; Anai N. Kothari, MD, MS ${ }^{\star *}$, Loyola University Chicago, Maywood, IL; Catherine M. Kuhn, MD, Duke University Health System, Durham, NC; Tanya Lord, PhD, MPH, Foundation for Healthy Communities, Concord, NH; David Markenson, MD, MBA, FAAP, FACEP, FCCM, New York Medical College, Valhalla, NY; David Mayer, MD, MedStar Institute for Quality \& Safety, Columbia, MD; Douglas E. Paull, MD, MS, FACS, FCCP, CHSE**, VA National Center for Patient Safety, Ann Arbor, MI; Lakshmana Swamy, MD, MBA**, Boston University \& VA Boston Healthcare, Boston, MA; Andrew M. Thomas, MD, MBA ${ }^{\star \star}$, Wexner Medical Center at The Ohio State University, Columbus, OH; Marjorie S. Wiggins, DNP, MBA, RN, FAAN, NEA-BC, Maine Medical Center, Portland, ME; Ronald Wyatt, MD, MHA, DMS(Hon), Cook County Health and Hospitals, Chicago, IL.

**Past CLER Evaluation Committee member 


\section{Detailed Findings}

Nancy J. Koh, PhD; Robin Wagner, RN, MHSA; Baretta R. Casey, MD, MPH, FAAFP; Robin C. Newton, MD, FACP; Hongling Sun, PhD; and Kevin B. Weiss, MD; on behalf of the CLER Program*

\section{INTRODUCTION}

This section includes detailed findings from the first set of visits (2015-2017) of the Clinical Learning Environment Review (CLER) Program in the 6 CLER Focus Areas ${ }^{1}$ to the major participating clinical sites (ie, hospitals, medical centers, and ambulatory settings) for 270 Accreditation Council for Graduate Medical Education (ACGME)-accredited Sponsoring Institutions (SIs) with 2 or fewer core residency programs. These clinical sites serve as clinical learning environments (CLEs) for the Sls. A separate report will be published on the data from the visits to the smallest sites of the Sls visited (ie, preventive medicine programs and single fellowship programs).

Collectively, the 270 Sls oversee 560 ACGME-accredited residency and fellowship programs. These smaller Sls account for $7.7 \%$ of all residents and fellows in ACGME-accredited programs-with a range of 1 to 144 trainees per SI (median=28).

Approximately $28 \%$ of the CLEs were located in the Southern region of the United States, $23.7 \%$ in the Northeast, $23.0 \%$ in the Midwest, and 22.2\% in the West. The sites ranged in size from 41 to 1111 acute care beds (median=295). The majority $(70.9 \%)$ were nongovernment, not-for-profit organizations; $12.4 \%$ were investor-owned, for-profit; $11.6 \%$ were government, nonfederal; and $5.2 \%$ were government, federal.

In total, the CLER Site Visit teams interviewed more than 1000 members of executive leadership (including chief executive officers), 1840 residents and fellows, 1789 core faculty members, and 459 program directors of ACGME-accredited programs in the group meetings. Additionally, the CLER teams interviewed the CLEs' leadership in patient safety and health care quality and thousands of residents, fellows, faculty members, nurses, pharmacists, social workers, and other health care professionals while on walking rounds in the clinical areas.

These findings are based on a mixed methods approach to data gathering and analysis to improve the accuracy of the findings by combining quantitative, descriptive, and qualitative evidence in a complementary manner (see Methodology, pp 15-22). As such, some of the findings are represented quantitatively while others are described qualitatively.

The combination of methodologies and varied representation of findings should be considered when interpreting the results, making comparisons, or drawing conclusions. Both supporting and conflicting evidence may be presented to explain or qualify findings. For example, results from the group interviews may appear more positive than information gathered on walking rounds. Alternatively, practices reported during group interviews may have been verified on walking rounds. 


\section{INTERPRETING QUANTITATIVE RESULTS FROM THE GROUP INTERVIEWS}

During the group interviews with residents and fellows, faculty members, and program directors, an electronic audience response system (ARS; Keypoint Interactive version 2.6.6, Innovision Inc, Commerce Township, MI) was used to collect anonymous responses to closed-ended questions. The results from the ARS were analyzed at both the individual (eg, residents and fellows) and the CLE levels.

At the individual level of analysis, results are presented as percentages of the total number of individuals surveyed. For example:

"In the group interviews, $46.6 \%$ of residents and fellows reported that they had received cultural competency training that was specific to populations at risk for health care disparities at their clinical site."

At the CLE level of analysis, individual responses were aggregated at the CLE level and results are presented as median and interquartile range (IOR) percentages. For example:

"Across CLEs, a median (IOR) of 50.0\% (37.5\%-66.7\%) of the residents and fellows indicated that they had received cultural competency training that was specific to populations at risk for health care disparities at their clinical site."

Statistically significant differences (ie, $P \leq .05$ ) in responses due to resident and fellow characteristics (eg, residency year) and CLE characteristics (eg, bed size) are also reported. Of note, statistical significance does not always imply practical significance. For example, differences in responses by residency year may be statistically significant but the differences may not be meaningful or large enough to have practical relevance or implications.

\section{ADDITIONAL CONSIDERATIONS}

As described in the Methodology section (pp 15-22), this report contains a specific set of descriptive terms that summarize quantitative results from both the ARS and specific findings that were quantified from the site visit reports. These terms and their corresponding quantitative ranges are as follows:

few $(<10 \%)$, some (10\%-49\%), most (50\%-90\%), and nearly all (>90\%)

Besides the quantitative data, this report contains qualitative data from a number of open-ended questions that CLER Site Visitors asked during group interviews and walking rounds. This information, by design, was not intended to be enumerated. For these questions, the site visit teams made an assessment of the relative magnitude of observations at each individual site. To prevent confusion, these results are presented in the report using a set of descriptive terms different from the previously described terms used for quantitative data. The qualitative descriptive terms, which are intended to approximate the quantitative terms above, are as follows:

uncommon or limited, occasionally, many, and generally

Finally, this section follows approximately the same structure as the individual CLER Site Visit reports received by participating institutions. This structure is intended to facilitate easy comparison between data from an individual site and that of this report, which aggregates results from all 270 Sls. Those who seek additional detail may consult the appendices (pp 87-130). 


\section{PATIENT SAFETY}

The CLER Program explored several aspects of resident and fellow engagement in patient safety with emphasis on 5 major topics: culture of safety, use of the patient safety event reporting system, knowledge of patient safety principles and methods, inclusion in patient safety event investigations, and disclosure of patient safety events. Generally across CLEs, members of the executive leadership team identified patient safety as their highest priority area for improvement.

\section{Culture of Safety}

The patient safety and quality leaders in many CLEs indicated that they periodically conduct a culture of safety survey that includes residents, fellows, and faculty members. Overall, $98.0 \%$ of the residents and fellows in the group interviews indicated that their CLE provides a safe and nonpunitive environment for reporting errors.

Across CLEs, physicians and other staff members also indicated use of the patient safety event reporting system to report on individual behaviors. This use included reporting on behaviors in a retaliatory fashion or in a manner that could be perceived as punitive.

Given this and based on the collective findings from the site visits, it is unclear as to whether residents, fellows, and other staff members perceived a safe and nonpunitive culture for reporting patient safety events.

\section{Use of the Patient Safety Event Reporting System}

\section{CLE Systems for Reporting}

Overall, CLEs had 1 or more mechanisms for reporting patient safety events, including an online or paperbased patient safety event reporting system, a chain-of-command system that allowed events to be reported to an immediate supervisor (eg, a more senior resident or faculty member), and a mechanism to verbally report events to the patient safety staff (eg, hotline).

In general, residents and fellows appeared to be aware of their CLE's process for reporting patient safety events such as adverse events, near misses/close calls, and unsafe conditions. During walking rounds, the CLER Site Visit teams also asked nurses about their CLE's patient safety event reporting system. Across nearly all CLEs (96.3\%), nurses appeared to be familiar with their CLE's system for reporting patient safety events.

Approximately 35\% of CLEs were able to provide information on the number of patient safety event reports submitted by residents and fellows (see Appendix C1), and $40.5 \%$ were able to provide the number of patient safety event reports submitted by attending physicians. The remaining CLEs indicated that their system did not track such information. Whereas CLEs occasionally provided the Graduate Medical Education Committee with information on the number or percentage of patient safety event reports submitted by residents and fellows, it was less common for them to routinely provide the same group with the number or percentage of patient safety event reports submitted by faculty members. Additionally, a limited number of CLEs provided their governing body with information on the number or percentage of patient safety event reports submitted by residents, fellows, and faculty members. 


\section{Understanding of Reportable Events}

Generally across CLEs, the residents and fellows interviewed on walking rounds appeared to lack understanding and awareness of the range of reportable patient safety events, including what defines a near miss/close call. In nearly all CLEs (96.5\%), nurses' understanding of reportable patient safety events also varied (see Appendix C2).

Across CLEs, residents, fellows, and nurses appeared to focus on reporting sentinel events, medication errors, patient falls, and other events with harm; they did not appear to recognize near misses/close calls, unsafe conditions, events without harm, unexpected deteriorations, or known procedural complications as reportable patient safety events. Residents, fellows, and nurses appeared to have little awareness of the importance of reporting these events and how such reporting can provide valuable information for identifying system failures, addressing vulnerabilities in the system, reducing risks, and improving patient safety.

\section{Reporting}

Overall, $66.7 \%$ of the residents and fellows in the group interviews indicated that they had experienced an adverse event, near miss/close call, or unsafe condition while at their CLE. This experience varied by year of training and specialty grouping (see Appendix B1).

Of the residents and fellows who reported that they had experienced an adverse event, near miss/close call, or unsafe condition, $56.5 \%$ indicated that they had personally reported the patient safety event using the CLE's patient safety event reporting system. Responses varied by gender and year of training. Across CLEs, the median (IOR) finding was 50.0\% (33.3\%-80.0\%) and varied by CLE bed size and type of ownership (see Appendix B2). For those who did not personally enter the patient safety event into the system, $9.7 \%$ indicated that they relied on a nurse or medical assistant to submit the patient safety event report, $24.9 \%$ indicated that they relied on a physician supervisor, and $8.9 \%$ indicated that they cared for the patient and chose not to submit a report.

When faculty members and program directors in the group interviews were asked what process residents and fellows most frequently followed when reporting a patient safety event, $61.4 \%$ of the faculty members and $49.6 \%$ of the program directors indicated that they believed residents and fellows most often reported the event themselves using the CLE's patient safety event reporting system.

In a separate query, $29.5 \%$ of the residents and fellows in the group interviews indicated that they had reported a near miss/close call event while at the CLE; responses varied by year of training and specialty grouping (Figure 1). Across CLEs, this finding ranged from $0 \%$ to $100 \%$, with a median (IQR) of $25.0 \%$ $(11.1 \%-48.6 \%)$; responses varied by CLE bed size and type of ownership (see Appendix B3). 


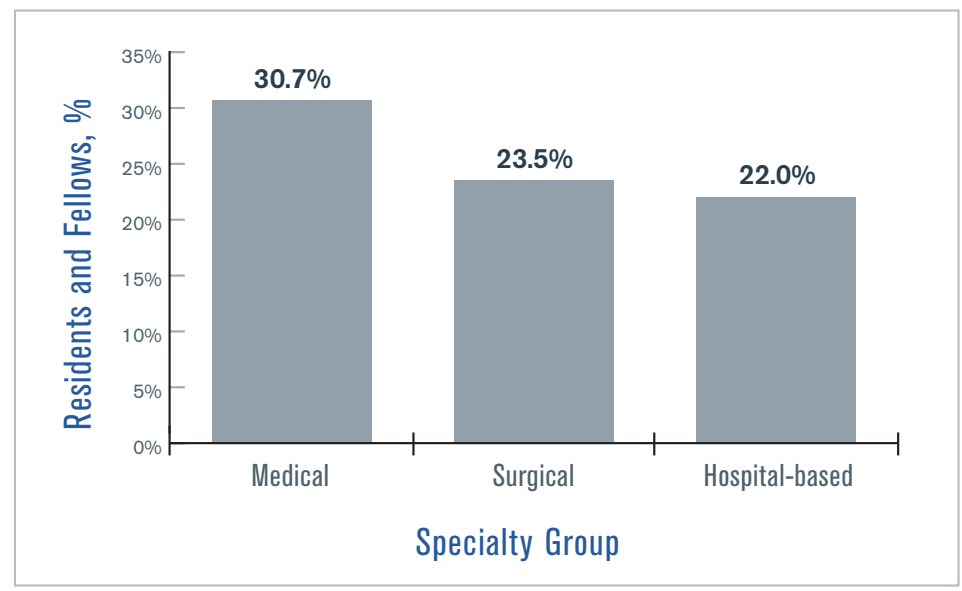

Figure 1. Percentage of Residents and Fellows Who Reported a Near Miss/Close Call Event, by Specialty Group

On walking rounds, residents and fellows in many CLEs mentioned that they often report patient safety events locally or through their chain of command while also indicating familiarity with the patient safety event reporting system and its use. When they delegated or relied on others to report, it was unclear if these reports were formally captured in the CLE's centralized patient safety event reporting system. Residents and fellows mentioned the cumbersome process of submitting a report, the time needed to enter a report, fears of repercussion, and the uncertainty of receiving feedback as reasons for not reporting. The collective information from the site visits indicated that in $\mathbf{8 2 . 8 \%}$ of the CLEs, resident and fellow reporting of patient safety events into the CLE's patient safety event reporting system was varied or infrequent (see Appendix C3).

In the group interviews, the CLER Site Visit teams also explored faculty members' and program directors' use of the CLE's patient safety event reporting system. Approximately $40 \%$ of the faculty members reported that they had personally reported an adverse event, near miss/close call, or unsafe condition in the past year (median [IQR], 40.0\% [25.0\%-55.1\%] across CLEs). Among the program directors, $46.0 \%$ reported that they had personally reported an adverse event, near miss/close call, or unsafe condition in the past year (4.4\% had no clinical responsibilities at the site). Across CLEs, the median (IQR) finding was $50.0 \%$ (0\%-100\%). In both groups, responses varied by region. 


\section{Feedback}

In the group interviews, the CLER Site Visitors asked residents and fellows whether they received feedback on patient safety event reports. Of those who had experienced an adverse event, near miss/close call, or unsafe condition and who had personally submitted a patient safety event report or relied on a nurse, medical assistant, or supervisor to submit the report, $54.1 \%$ reported that they received feedback on the outcome of the report. Responses varied by gender (Figure 2; see also Appendix B4).

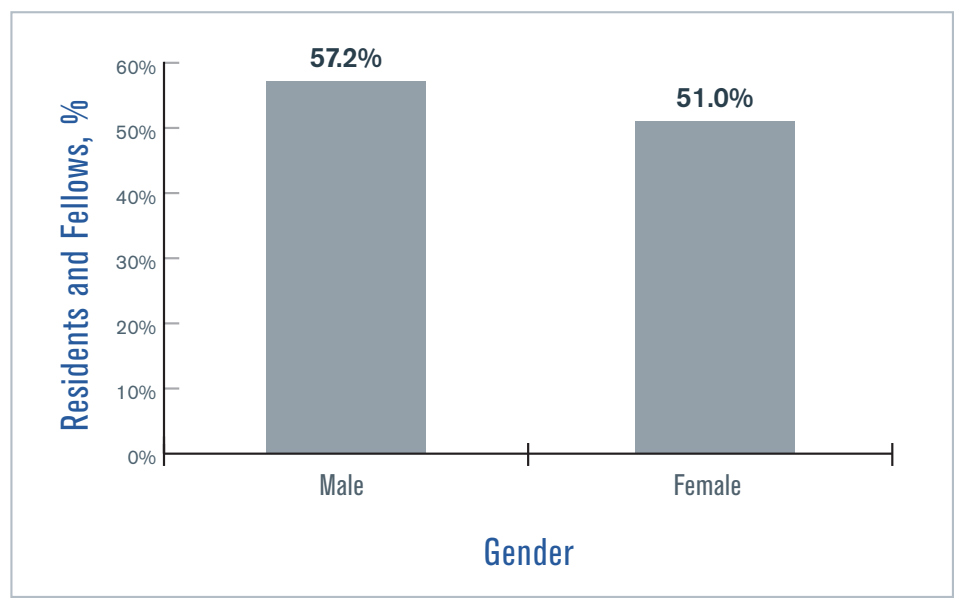

Figure 2. Percentage of Residents and Fellows Who Reported Receiving Feedback on the Outcome of a Patient Safety Event Report Submitted, by Gender

Residents and fellows often mentioned receiving an e-mail acknowledging receipt of the patient safety event report. They also noted receiving requests for additional information as part of a formal patient safety event investigation. It was uncommon for residents to mention receiving information on the outcome of the investigation, including recommended actions to address vulnerabilities in the system and to improve patient safety. Across CLEs, residents, fellows, nurses, and other clinical staff expressed a strong desire to receive feedback in response to submitting a patient safety event report.

Overall, CLEs varied in their processes for reviewing and prioritizing patient safety events. Residents and fellows also varied in their knowledge of these processes and often indicated that these processes were unknown or unclear. Many residents and fellows appeared to be unaware of how their CLEs use the reporting of adverse events, near misses/close calls, or unsafe conditions to improve care both broadly and at the individual departmental level. Residents and fellows were rarely involved in their CLE's process for reviewing and prioritizing patient safety events that required further investigation.

\section{Time-Outs}

On walking rounds, the CLER Site Visit teams explored resident and fellow participation in the time-out process as part of patient safety practices (eg, ambulatory and bedside procedures). Across many CLEs, residents, fellows, nurses, and other health care professionals interviewed on walking rounds indicated that residents and fellows do not consistently conduct standardized time-outs before performing bedside procedures. 


\section{Knowledge of Patient Safety Principles and Methods}

Across most CLEs (88.5\%), residents and fellows appeared to have limited knowledge of fundamental patient safety principles and methods (eg, Swiss cheese model of system failure, root cause analysis, fishbone diagrams; see Appendix C4).

When asked to identify their skills in applying patient safety principles, the majority of the faculty members indicated that they were either proficient or expert (67.5\% and $22.2 \%$, respectively) in applying these skills. Similarly, most of the program directors reported themselves as proficient or expert $(70.3 \%$ and $18.3 \%$, respectively).

Of the residents and fellows in the group interviews, $44.2 \%$ reported that they had participated in a structured interprofessional simulation activity related to patient safety. Responses varied by year of training and specialty grouping. Across CLEs, the median (IQR) finding was 40.0\% (22.2\%-66.7\%), with responses varying by CLE bed size and type of ownership.

\section{Inclusion in Patient Safety Event Investigations}

In many CLEs, the patient safety and quality leaders indicated that they did not track resident and fellow participation in patient safety event investigations (eg, root cause analysis). A limited number of CLEs provided the Graduate Medical Education Committee and the governing body with information regarding the number of residents and fellows who had participated in formal patient safety event investigations.

The CLER Site Visitors also asked the program directors in the group interviews if they measured resident and fellow participation in patient safety event investigations. Approximately $48 \%$ of the program directors reported tracking resident and fellow involvement (median [IOR], 50.0\% [0\%-100\%] across CLEs). Responses varied by type of ownership.

In the group interviews, $39.8 \%$ of the residents and fellows indicated that they had participated in an interprofessional investigation of a patient safety event that included components such as analysis of system issues, development and implementation of an action plan, and monitoring for continuous improvement.

Reponses varied by year of training (Figure 3). Across CLEs, the median (IOR) finding was 37.5\% (20.0\%$60.0 \%$ ), with responses varying by region and CLE bed size (see Appendix B5).

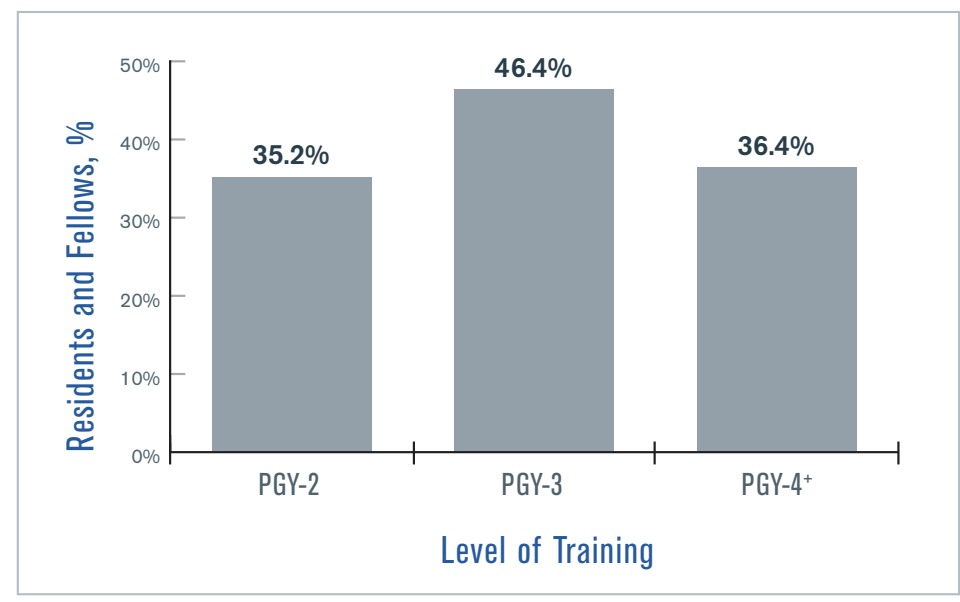

Figure 3. Percentage of Residents and Fellows Who Reported Participating in an Interprofessional Patient Safety Investigation, by Level of Training

Abbreviation: PGY, postgraduate year 
The CLER Site Visitors also asked faculty members about their involvement in interprofessional patient safety event investigations. Approximately $63 \%$ of the faculty members in the group interviews reported that they had participated in an investigation of a patient safety event that involved physicians, nurses, administrators, and other health care professionals (median [IOR], 66.7\% [42.9\%-80.0\%] across CLEs).

Overall, the format and process of investigating patient safety events varied both across and within CLEs. It was uncommon for residents and fellows to describe involvement in comprehensive systems-based approaches to patient safety event investigations aimed at preventing future adverse events and sustaining improvements in patient safety. In general, residents and fellows described experiences that lacked the attributes of a formal patient safety event investigation, with very little or no interprofessional or interdisciplinary engagement. Residents and fellows varied widely in their perceptions of what constituted a formal investigation of a patient safety event. Across many CLEs, case conferences, morbidity and mortality conferences, and grand rounds continued to be the major approach to patient safety event investigations.

Faculty members and program directors indicated that departmental morbidity and mortality conferences, case conferences, and online modules were other informal approaches to model elements of a patient safety event investigation.

\section{Disclosure of Patient Safety Events}

In the group interviews, $76.3 \%$ of the residents and fellows indicated that they had received training on disclosing medical errors to patients and/or families (2.2\% reported that such training was not applicable). Responses varied by year of training and specialty grouping. Across CLEs, the median (IQR) finding was $80.0 \%(64.4 \%-100 \%)$. Of those who received training, $7.9 \%$ indicated that the training was primarily simulation based; $71.1 \%$, didactic and/or online; $12.0 \%$, informal; and 9.0\%, other.

Approximately $89 \%$ of the residents and fellows in the group interviews indicated that they knew of CLE resources to assist them in coping with a major patient safety event that resulted in a patient death or harm (median [IQR], 100\% [82.0\%-100\%] across CLEs; see Appendix B6 for information on variability). Of those familiar with the resources, most indicated that they would be somewhat $(31.5 \%)$ or very comfortable $(55.5 \%)$ in using these resources. 


\section{HEALTH CARE QUALITY (INCLUDING HEALTH CARE DISPARITIES)}

The CLER Program explored resident and fellow engagement in improving health care quality within the context of 6 major areas: involvement in developing and implementing the CLE's strategies for health care quality, awareness of the CLE's health care quality priorities, knowledge of health care quality terminology and methods, engagement in quality improvement (OI) projects, access to quality metrics data, and engagement in CLE efforts to address health care disparities.

\section{Involvement in Developing Health Care Quality Strategies}

As part of understanding the CLE's approach to improving health care quality, the CLER Site Visit teams reviewed the organization's strategic plan for quality and interviewed both executive and patient safety and quality leaders. Overall, a limited number of CLEs appeared to integrate QI within the organization as part of a system-wide, comprehensive approach to promote experiential learning and to improve quality and safety across the organization.

Across CLEs, resident and fellow involvement in strategic planning for Ql was uncommon. Residents and fellows often served as implementers of CLE-wide Ql activities (eg, hand hygiene, reducing hospital-acquired infections, reducing 30-day readmissions).

A limited number of CLEs had instituted resident and fellow committees aimed at increasing resident and fellow engagement in Ql; few of these committees were integrated into the CLE's formal Ql processes. In many CLEs, resident and fellow participation in institutional $\mathrm{Ql}$ committees was uncommon; often, roles and expectations for participation were undefined or unclear. The clinical sites also appeared to have insufficient structure to allow residents and fellows to attend committee meetings regularly and to participate in meaningful ways. Additionally, residents and fellows in many CLEs were not included in the governing body's patient safety and quality committees.

\section{Priorities for Improving Health Care Quality}

In general, priorities for improving health care quality varied across CLEs. However, some common themes included alignment with broad national priorities such as Core Measures or publicly reported performance measures (eg, diabetic management, hand hygiene, hospital-inquired infections). Many CLEs were also highly focused on meeting specific criteria such as reducing 30-day readmissions or improving performance on metrics related to pneumonia, chronic heart failure, and surgical care improvement project measures.

In the group interviews, $86.3 \%$ of the residents and fellows (postgraduate year 2 and above) reported knowing their CLE's priorities for improving health care quality (see Appendix B7 for additional information on variability). When asked the same question, $86.7 \%$ of the faculty members and $91.2 \%$ of the program directors reported knowing the priorities. Often, the physician groups focused on departmental activities and did not describe priorities that aligned with those identified by the CLE's executive leadership or the patient safety and quality leaders. When the physicians identified priorities aligned with those of executive leadership, they were most commonly around nationally recognized measures, especially those related to programs with financial incentives. 


\section{Knowledge of Health Care Quality Improvement}

In 51.5\% of the CLEs, the residents and fellows appeared to have limited knowledge or understanding of basic Ol terminology and methods such as Lean, Plan-Do-Study-Act, and Six Sigma (Figure 4, see also Appendix C5). A limited number of residents and fellows could articulate the $\mathrm{Ol}$ approach employed by their CLE in designing and implementing $\mathrm{Ql}$ activities to improve patient care.

In general, the approach to educating residents and fellows about health care Ol varied both within and between CLEs. Although some type of education was common as part of new resident and fellow orientation, a limited number of CLEs aimed to provide ongoing training for all residents and fellows. Training in health care Ql appeared to occur primarily within departments or graduate medical education (GME) programs, and the format, methods, and content appeared to vary widely.

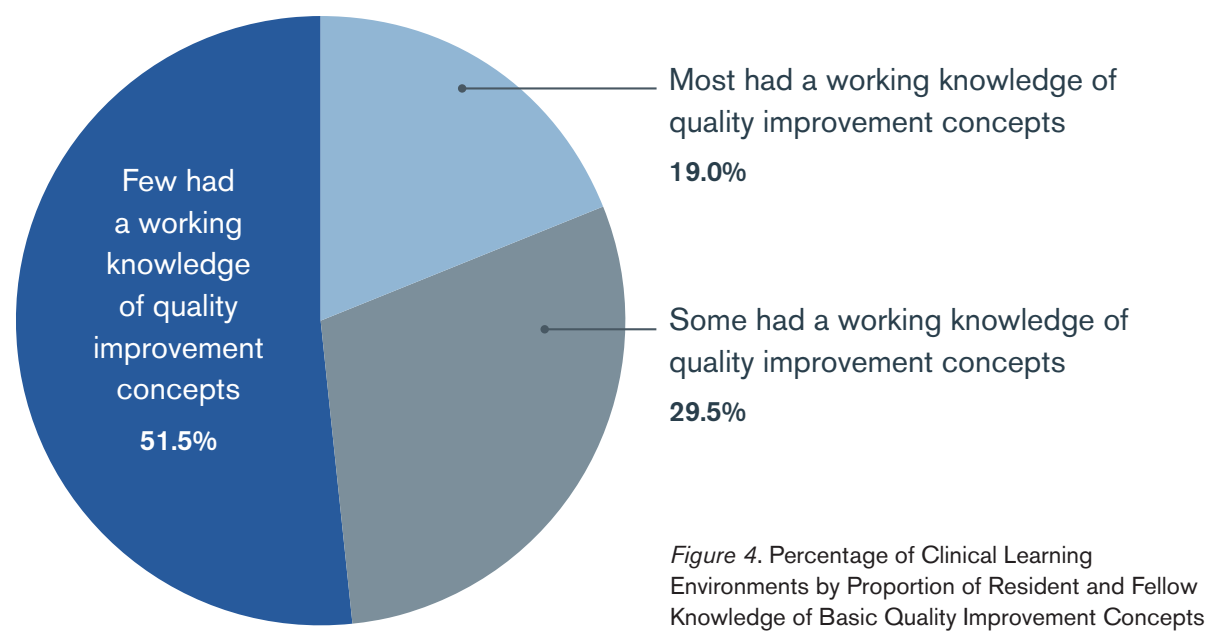

\section{Engagement in Quality Improvement Projects}

In $15.2 \%$ of the CLEs, the patient safety and quality leaders indicated that they monitor resident and fellow QI projects.

In the group interviews with residents and fellows (postgraduate year 2 and above), 80.3\% reported they had participated in a Ql project of their own design, or one designed by their program, department, or ambulatory care setting. Of this group, $60.7 \%$ reported that their Ql project was directly linked to 1 or more of the CLE's goals; $18.1 \%$ were uncertain. Of those who reported that their Ol projects were linked to the CLE's goals, 83.4\% reported that their projects involved interprofessional teams. Appendices B8, B9, and B10 provide detailed information on variability.

In the group interviews and on walking rounds, the CLER Site Visitors asked residents and fellows to describe their QI projects. Overall, residents and fellows varied in their descriptions of these projects. It was uncommon for residents and fellows to describe projects that aligned with their CLE's priorities. In most CLEs (78.3\%), few described projects that included the components of a complete Ol cycle (ie, Plan-Do-Study-Act) (Figure 5; see also Appendix C6). Often, resident and fellow participation was limited to planning and implementing a Ql 
activity. For many residents and fellows, their Ql projects did not involve formally assessing effectiveness and designing follow-up actions to adjust, support, and sustain ongoing Ql efforts.

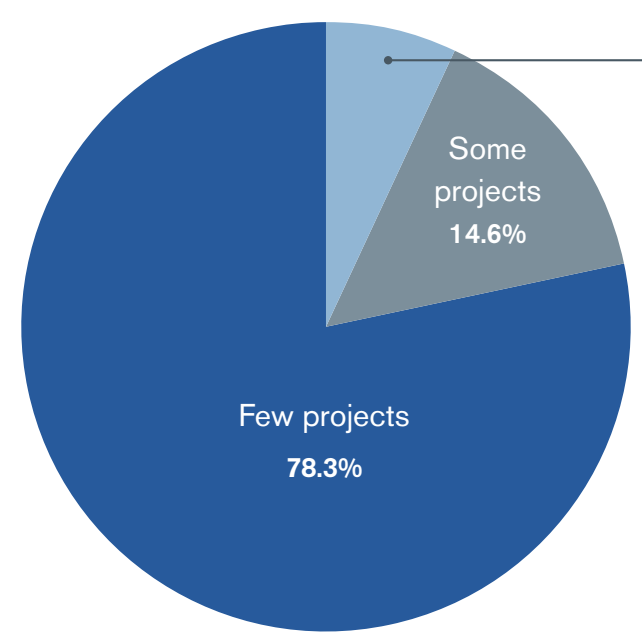

Most projects

$7.1 \%$

Figure 5. Percentage of Clinical Learning

Environments by Proportion of Resident and Fellow

Quality Improvement Projects With Components of

a Complete Quality Improvement Cycle

It was also uncommon for residents and fellows to describe involvement in interprofessional team-based Ql projects. During the interviews on walking rounds, a limited number of nurses and other health care professionals indicated that they were involved in interprofessional Ql projects that included residents and fellows.

When the CLER Site Visitors queried faculty members in the group interviews about their engagement in interprofessional Ql projects, $74.8 \%$ reported that they had participated in a Ol project with nurses, pharmacists, and other members of the health care team (median [IOR], 77.8\% [62.5\%-100\%] across CLEs).

\section{Access to Data}

In the group interviews, $79.9 \%$ of the program directors reported that their residents and fellows have ready access to organized systems for collecting and analyzing data for the purposes of Ql. Electronic health records, specialty-specific clinical registries, and local, regional, or national quality dashboards were often reported as common sources of QI data. Residents and fellows often mentioned the challenges (eg, long waiting lists) in acquiring specific reports from these data sources. Many faculty members noted that residents and fellows had limited support for data analysis. When support existed, it was often a departmental resource. The type and extent of analytic support services available to residents and fellows varied both within and across CLEs.

Overall, $53.2 \%$ of the residents and fellows in the group interviews reported receiving aggregated or benchmarked QI data on their own patients. Responses varied by year of training and specialty grouping. Across CLEs, the median (IQR) finding was 55.6\% (33.3\%-80.0\%), with responses varying by type of ownership (Figure 6; see also Appendix B11). 


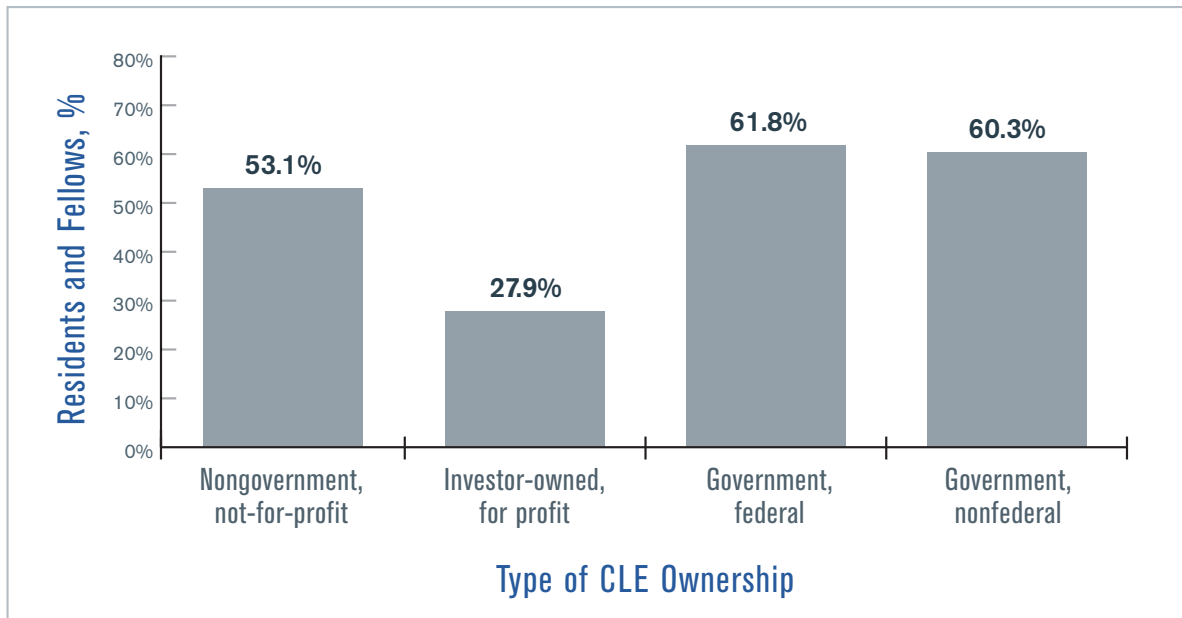

Figure 6. Percentage of Residents and Fellows Who Reported Receiving Aggregated or Benchmarked Quality Performance Data About the Care of Their Own Patients, by Type of Clinical Learning Environment (CLE) Ownership

Occasionally, the patient safety and quality leaders indicated that residents and fellows receive Ql data to compare the care of their own patients with others served by their clinical site.

\section{Engagement in CLE Efforts to Address Health Care Disparities Strategies and Priorities in Health Care Disparities}

Across many CLEs, executive leaders were aware of issues of health disparities affecting their surrounding communities. Many described conducting community needs assessments and improving access to care by providing free or low-cost care and clinics for the underserved, often staffed by residents and fellows from a few core specialties (eg, family medicine, internal medicine, pediatrics, obstetrics and gynecology). A limited number of residents and fellows from other specialty and subspecialty programs reported engaging in these activities.

A limited number of executive leaders spoke to health care disparities occurring within their hospital or medical center. Overall, less than $5 \%$ of executive leaders described a specific set of strategies or a systematic approach to identifying, addressing, and continuously assessing variability in the care provided to or the clinical outcomes of their patient populations at risk for health care disparities. In some of the CLEs, the executive leaders, faculty members, or program directors indicated that some departments were collecting data or conducting studies related to health care disparities among specific patient populations; many of these efforts were reported as research projects.

In the group interviews, $65.7 \%$ of the residents and fellows reported that they knew their CLE's priorities in addressing disparities in health care; responses varied by gender and specialty grouping. Across CLEs, this finding ranged from $0 \%$ to $100 \%$ (median $[I \mathrm{IQR}], 73.0 \%$ [50.0\%-88.5\%]). Responses varied by region, CLE bed size, and type of ownership (see Appendix B12). In comparison, $71.6 \%$ of the faculty members and $76.0 \%$ of the program directors reported that they knew their CLE's priorities with regard to health care disparities.

\section{Cultural Competency}

Overall, residents, fellows, faculty members, and program directors interviewed in the group interviews were able to describe populations at risk for health care disparities at their clinical site. 
In the group interviews, $46.6 \%$ of the residents and fellows reported that they had received cultural competency training that was specific to populations at risk for health care disparities at their clinical site, $25.0 \%$ reported receiving training that was not specific to the CLE's patient population, $24.9 \%$ reported receiving training that was primarily informal while providing clinical care, and 3.5\% indicated that they had not received cultural competency training at their CLE.

Across CLEs, a median (IOR) of 50.0\% (37.5\%-66.7\%) of the residents and fellows indicated that they had received cultural competency training that was specific to populations at risk for health care disparities at their clinical site. Responses varied by region and type of ownership (Figure 7; see also Appendix B13).

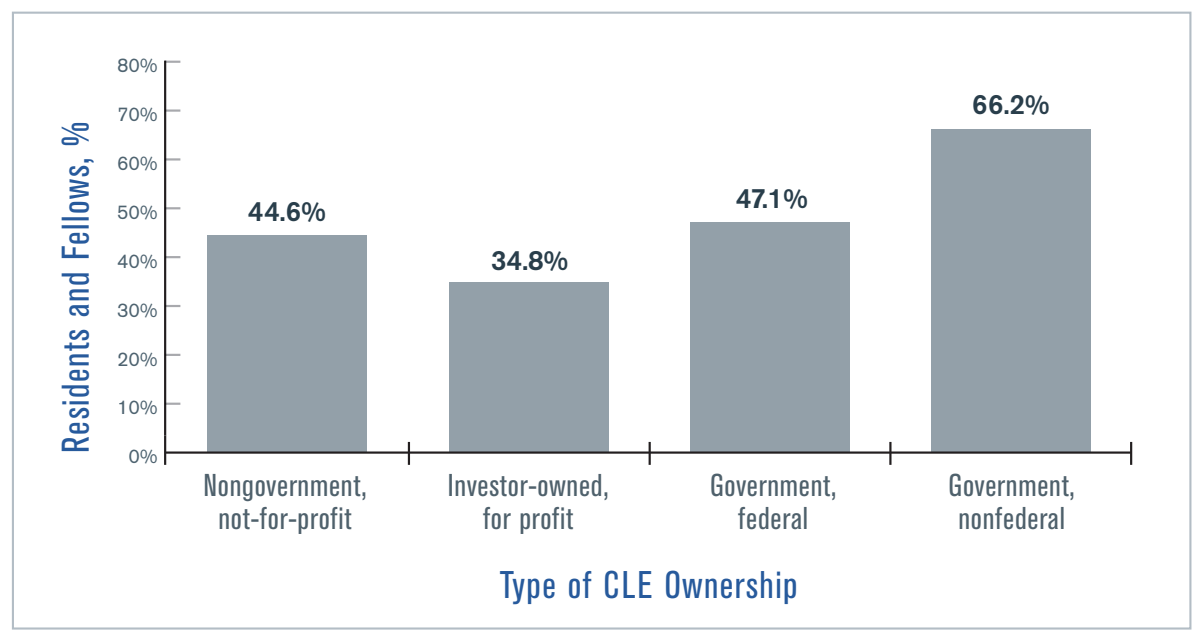

Figure 7. Percentage of Residents and Fellows Who Reported Receiving Cultural Competency Training Specific to Populations at Risk for Health Care Disparities at Their Clinical Site, by Type of Clinical Learning Environment (CLE) Ownership

During interviews on walking rounds, many residents and fellows described education and training in cultural competency that was largely generic and not specific to the diverse populations receiving care at their clinical site. In general, residents and fellows across CLEs indicated receiving informal training in cultural competency while delivering care.

In the group interviews, $39.2 \%$ of faculty members reported that they had received cultural competency training that was specific to populations at risk for health care disparities and receiving care at their clinical site. Approximately $30 \%$ reported receiving training that was basic and not specific to the CLE's patient population, 23.2\% reported receiving training that was primarily informal, and $7.4 \%$ reported that they had not received cultural competency training at the CLE.

\section{Participation in Quality Improvement to Address Health Care Disparities}

Overall, $23.6 \%$ of the faculty members in the group interviews indicated receiving periodic reports of their CLE's patient outcomes related to health care disparities (median [IOR], 16.7\% [0\%-37.5\%] across CLEs). Of those who reported receiving patient outcomes data related to health care disparities, $59.7 \%$ indicated that their residents and fellows use the data to reduce disparities in health care (median [IQR], 50.0\% [0\%-100\%] across CLEs). 


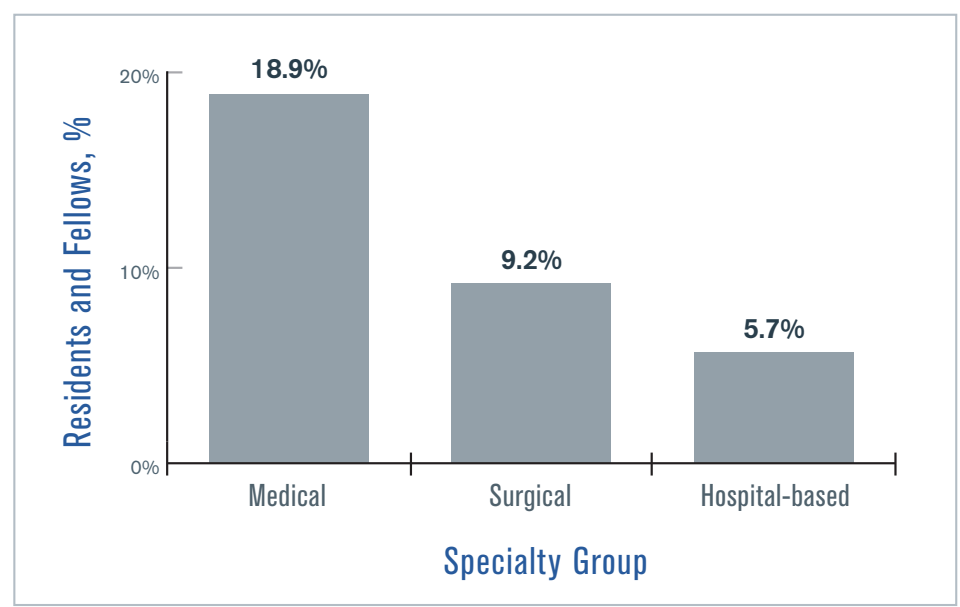

Figure 8. Percentage of Residents and Fellows Who Reported Participating in a Quality Improvement Project Focused on Reducing Health Care Disparities, by Specialty Group

Approximately $17 \%$ of the residents and fellows in the group interviews reported that they had participated in a QI project focused on reducing health care disparities; responses varied by gender, year of training, and specialty grouping (Figure 8; see also Appendix B14). In group discussions and on walking rounds, residents and fellows indicated that they rarely received CLE data related to health care disparities. 


\section{CARE TRANSITIONS}

The CLER Site Visitors explored several aspects of resident and fellow engagement in improving care transitions, including: priorities for improving care transitions, perceived vulnerabilities in care transitions, education on care transitions, inpatient transition processes, change-of-duty transitions, and supervision and assessment of care transitions.

\section{Priorities for Improving Care Transitions}

In describing priorities for improving transitions of care, many executive leaders focused on improving patient transfers from inpatient to postacute care (eg, rehabilitation centers, skilled nursing facilities, hospices) or transitions out of the hospital. A limited number of executive leaders mentioned improving provider-to-provider communications at change of duty (including resident and fellow handoffs) as a priority.

Occasionally, the executive leaders described efforts to create a standardized, organization-wide approach to 1 or more types of care transition. When they spoke about a standardized approach, they frequently mentioned using tools such as the I-PASS (ie, illness severity, patient summary, action list, situation awareness and contingency planning, and synthesis by receiver) model for handoffs or the SBAR (ie, situation, background, assessment, recommendation) technique.

Residents and fellows were often involved in efforts in designing, implementing, and standardizing their program's processes for shift-to-shift transitions of care. Faculty members were also involved in supporting residents and fellows in these efforts.

\section{Perceived Vulnerabilities in Care Transitions}

Across CLEs, residents, fellows, nurses, and other clinical staff identified many transitions that they believed posed vulnerabilities in patient safety. The executive leaders mentioned these same vulnerabilities. Examples included transfers from the emergency department to inpatient floors or units, transfers from the intensive care units to these floors, transfers from and to outside facilities, transfers from inpatient care back to the community, and multiple intrahospital transitions such as service to service and floor to floor, as well as between levels of care. Residents, fellows, and nurses often expressed concerns that communication during these transitions was incomplete or inaccurate, leading to vulnerability for patient safety events.

\section{Education on Care Transitions}

Overall, $75.8 \%$ of the residents and fellows reported that they had participated in training with nurses and other health care professionals on transitioning patient care. Responses varied by specialty grouping (median [IQR], $80.0 \%$ [60.0\%-100\%] across CLEs), as well as by region, CLE bed size, and type of ownership (see Appendix B15).

Across CLEs, standardized, organization-wide approaches to training in and managing care transitions between clinical services assigned to resident and fellow physician teams (eg, emergency department to inpatient care, operating room to intensive care unit) varied.

\section{Inpatient to Outpatient Transitions}

When queried, $81.6 \%$ of the residents and fellows in the group interviews indicated that they use a standardized process for transitioning patients from inpatient to outpatient care. ${ }^{a}$ Across CLEs, the median (IQR) was 87.5\% $(66.7 \%-100 \%)$, with responses varying by CLE bed size and type of ownership (Appendix B16).

${ }^{a}$ Results from visits that were held exclusively in the ambulatory care setting ( $9 \%$ ) have been omitted due to modifications to the question and/or scenario to better fit the setting. These modifications limited comparability across inpatient and ambulatory care settings. 
Interprofessional rounds varied across and within CLEs. These types of rounds were most commonly reported to occur in the labor and delivery and intensive care units or when planning for transitioning patients out of the hospital.

\section{Change-of-Duty Transitions}

Approximately $95 \%$ of the residents and fellows in the group interviews reported that they followed a standardized process for change-of-duty handoffs. ${ }^{a}$ Across CLEs, this finding ranged from $50.0 \%$ to $100 \%$. Of the residents and fellows who reported following a standardized process between shifts, $88.1 \%$ reported using a standardized written template of patient information to facilitate the handoff process. ${ }^{a}$ Across CLEs, the median (IOR) finding was 100\% (80.0\%-100\%), with responses varying by region and CLE bed size (Figure 9). Appendices B17 and B18 provide complete information on variability.

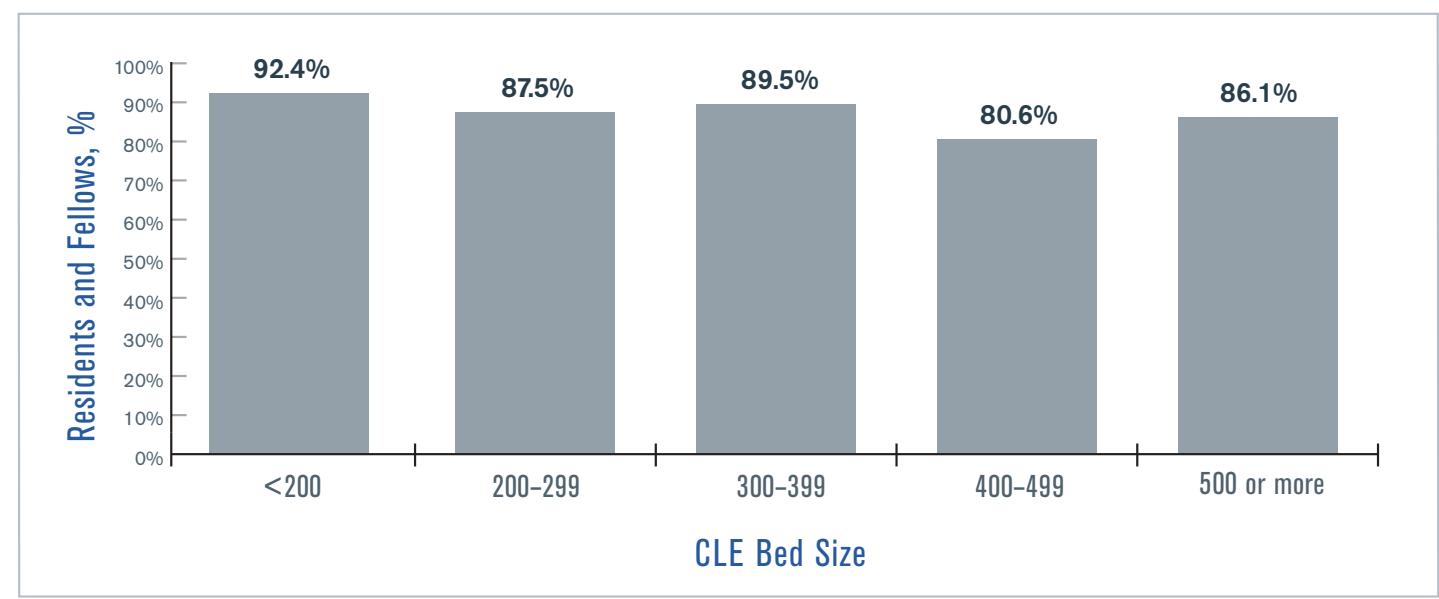

Figure 9. Percentage of Residents and Fellows Who Reported Following a Standardized Process for Handoffs Between Shifts and Using a Standardized Written Template, by Clinical Learning Environment (CLE) Bed Size

When asked how often attending physicians supervise their shift-to-shift handoff process, $32.0 \%$ of the residents and fellows in the group interviews reported that this supervision occurs daily, whereas $28.0 \%$ reported that it rarely occurs. ${ }^{a}$ Other responses included once per week (19.6\%), once per month (10.7\%), and less than once per month (9.8\%).

In group interviews, $73.3 \%$ of faculty members reported that they assess resident and fellow readiness to move from direct to indirect supervision in conducting change-of-duty handoffs (15.6\% reported that they do not conduct change-of-duty handoffs in their program). ${ }^{a}$ Of those who reported assessing resident and fellow readiness, $48.7 \%$ indicated that they assessed by direct observation only, $5.1 \%$ used a standardized assessment tool only, $42.9 \%$ used both direct observation and a standardized assessment tool, and 3.3\% used neither. ${ }^{a}$

Approximately $75 \%$ of the program directors in the group interviews indicated that their program assesses resident and fellow readiness to move from direct to indirect supervision in conducting change-of-duty handoffs (10.6\% reported that their program does not conduct change-of-duty handoffs). ${ }^{a}$ For those who indicated that their program assesses resident and fellow readiness, $45.4 \%$ indicated that their program assessed by direct

a Results from visits that were held exclusively in the ambulatory care setting (9\%) have been omitted due to modifications to the question and/or scenario to better fit the setting. These modifications limited comparability across inpatient and ambulatory care settings. 
observation only, $1.0 \%$ used a standardized assessment tool only, $51.1 \%$ used both direct observation and a standardized assessment tool, and $2.6 \%$ used neither. ${ }^{\mathrm{a}}$

From the information obtained on walking rounds, it appeared that the processes for transitioning care at change of duty vary widely across programs in most CLEs (91.9\%) (Figure 10; see also Appendix C7).

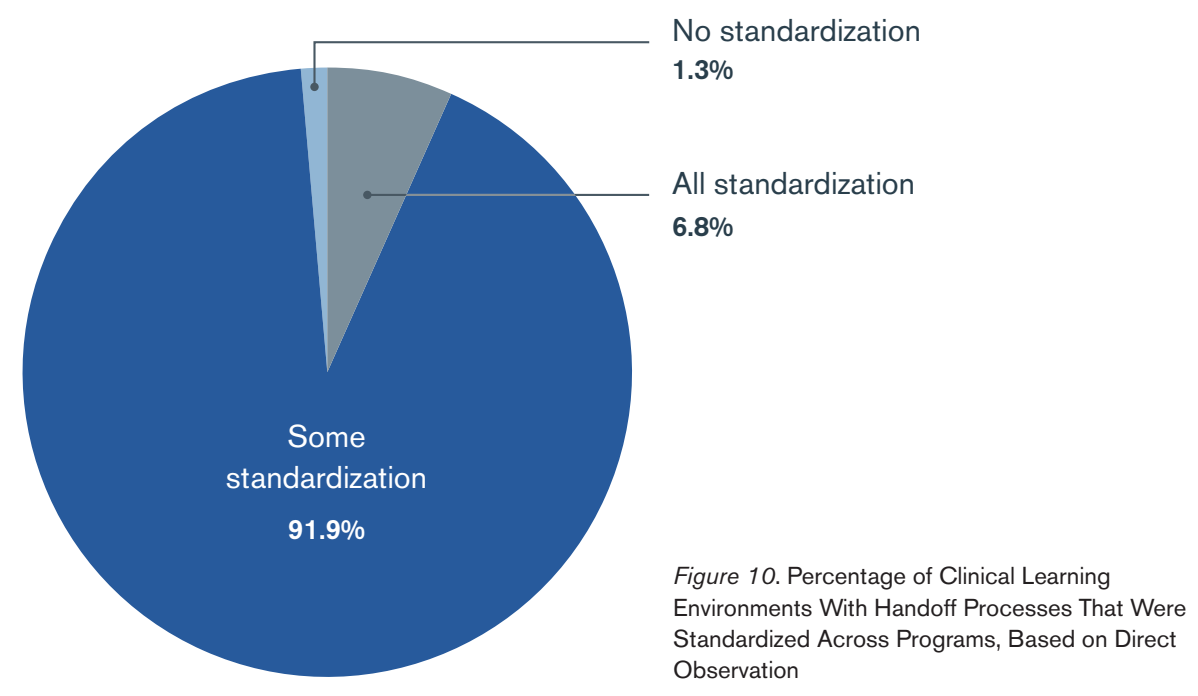

In general, the CLER Site Visit teams observed resident and fellow change-of-duty handoffs for 1 to 2 programs per visit. Overall, the handoffs varied both within and across CLEs. Many were conducted in quiet, nonpatient areas with minimal interruption. The handoffs varied in the use of written templates and other common tools for formal communication. Generally, faculty members were not consistently engaged in supervising these handoffs, and interprofessional involvement was uncommon. Contingency planning ("if-then" scenarios) and read-back techniques to confirm the plan of care were also inconsistent.

\section{Monitoring Care Transitions}

The patient safety and quality leaders in many CLEs indicated that they analyze patient safety event reports for issues related to resident and fellow transitions of care; they seldom mentioned active monitoring of these care transitions. 


\section{SUPERVISION}

The CLER Program explored resident and fellow supervision and the issues around this focus area for perceptions of supervision and potential vulnerabilities, awareness of situations requiring direct supervision, and patient safety events related to supervision.

\section{Perceptions of Supervision and Potential Vulnerabilities}

Across CLEs, many executive leaders did not express concerns or identify any specific vulnerabilities related to resident and fellow supervision within their organization. In general, residents, fellows, faculty members, and program directors also reported a culture of adequate supervision. When asked to summarize their experience at their CLE, $76.0 \%$ of the residents and fellows in the group interviews reported being adequately supervised. Most of the faculty members $(69.6 \%)$ and program directors $(72.5 \%)$ also indicated that residents and fellows are adequately supervised.

Although the majority of the physicians in the group interviews reported a culture of close supervision, they also reported perceptions of inadequate supervision. In the group interviews, $27.6 \%$ of the residents and fellows reported that while in training at the CLE, they had been placed in a situation or witnessed one of their peers in a situation where they believed supervision was inadequate (eg, the attending physician was not available). Responses varied by gender, specialty grouping, and level of training (Figure 11). Across CLEs, the median (IQR) finding was $20.0 \%$ (0\%-40.0\%), with responses varying by region and type of ownership (Appendix B19).

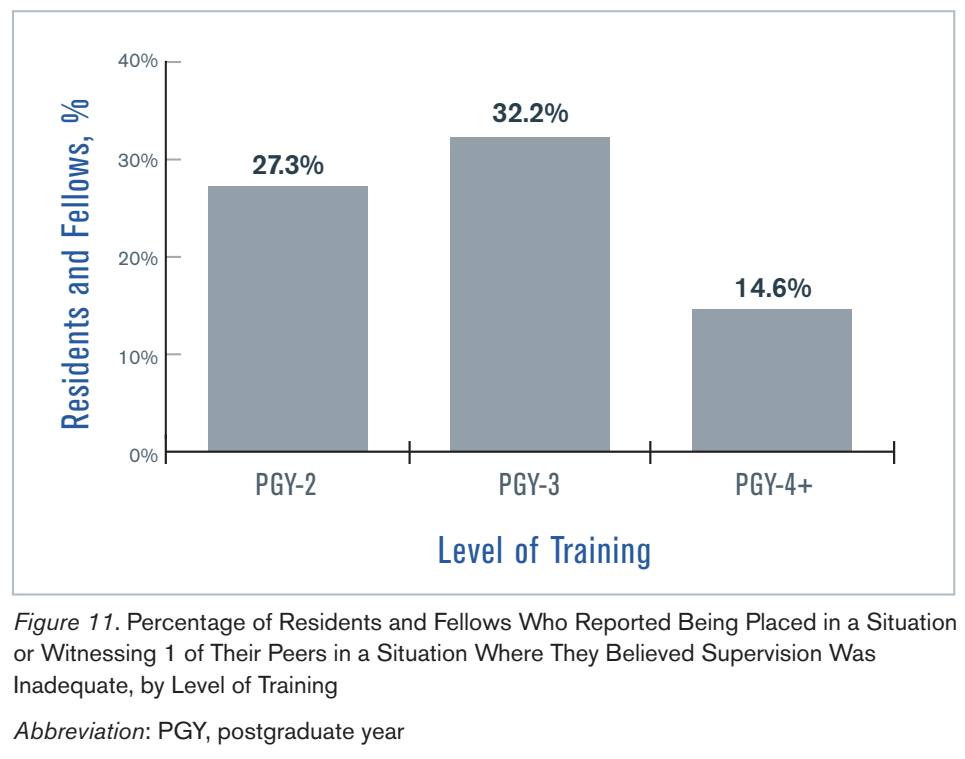

When asked about their experiences in contacting attending physicians and consultants for assistance, $50.1 \%$ of the residents and fellows in the group interviews indicated that they had encountered an attending physician or consultant who made them feel occasionally or frequently uncomfortable when requesting help at their CLE. Across CLEs, the median (IOR) finding was 50.0\% (25.0\%-75.0\%); responses varied by region, CLE bed size, and type of ownership (Figure 12; see also Appendix B20). Nearly $71 \%$ of the faculty members and $65.9 \%$ of the program directors perceived that their residents and fellows had encountered an attending physician or consultant who made them feel occasionally or frequently uncomfortable in requesting help. 


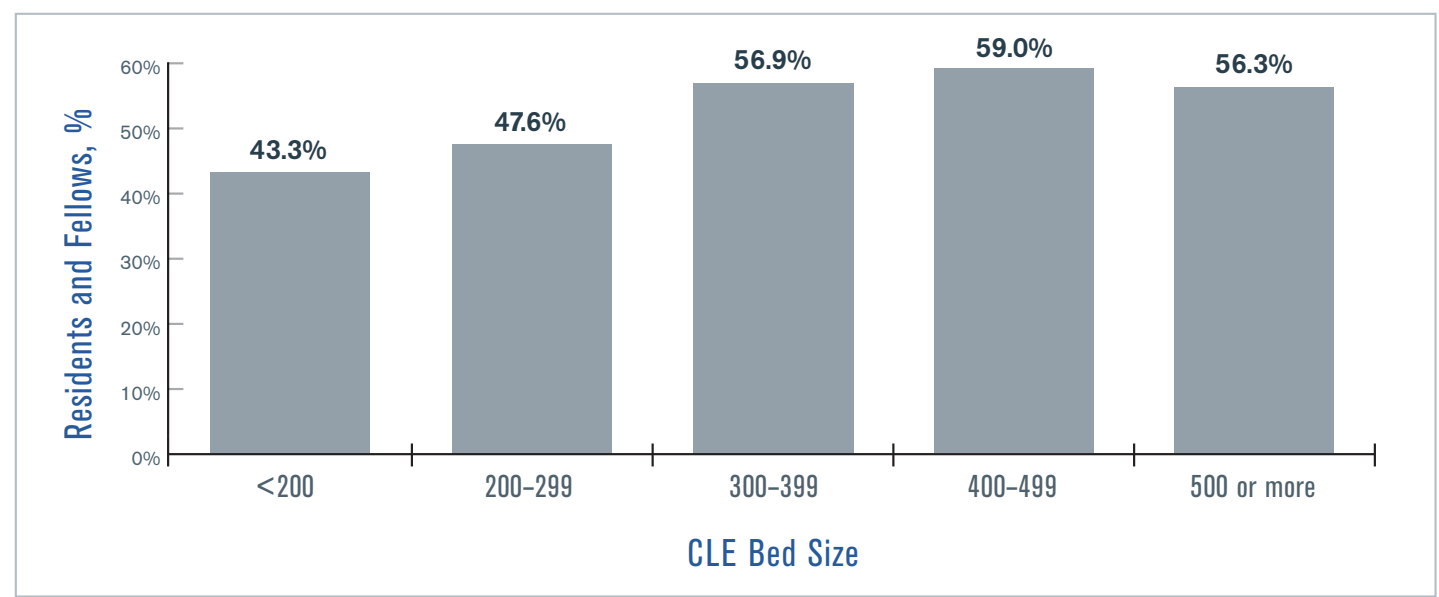

Figure 12. Percentage of Residents and Fellows Who Reported Encountering a Physician (Attending Physicians or Consultants) Who Made Them Feel Occasionally or Frequently Uncomfortable When Requesting Assistance, by Clinical Learning Environment (CLE) Bed Size

In discussing issues related to supervision that may be creating patient safety vulnerabilities, the physician groups frequently mentioned the challenges of providing supervision in the evenings, on weekends, and during times of high acuity and patient volume. They noted that in these situations, the number of faculty members was insufficient for adequate supervision. They also noted that competing clinical responsibilities further limited the availability of faculty members to supervise residents and fellows.

Residents and fellows mentioned gaps in supervision when their peers provide consultative services, noting these gaps as a potential source of patient safety vulnerabilities. Across CLEs, many residents and fellows expressed hesitancy to request help from attending physicians, noncore faculty, and consultants or to report concerns regarding supervision. They noted reluctance or discomfort to ask for assistance due to unwillingness to appear unprepared, a lack of understanding of when to escalate concerns in a timely manner, fear of retaliation or other negative consequences, and/or potential resistance when asking for help.

In addition to concerns of undersupervision, faculty members and program directors expressed concerns related to oversupervision, particularly in the procedural specialties, and the impact on resident and fellow readiness for independent practice. In discussing the factors that contribute to oversupervision, faculty members and program directors often mentioned the clinical site's policies on resident supervision and billing requirements of the Centers for Medicare \& Medicaid Services.

\section{Awareness of Situations Requiring Direct Supervision}

Overall, $97.0 \%$ of the residents and fellows in the group interviews reported that they knew what they were allowed to do without direct supervision. Responses varied by gender, level of training, and specialty grouping (Appendix B21).

In a separate query, $39.6 \%$ of the residents and fellows in the group interviews indicated that they had an objective way to know what procedures their peers from other services were allowed to do without direct supervision when providing consultative services on their patients $(36.8 \%$ reported that their peers from

${ }^{a}$ Results from visits that were held exclusively in the ambulatory care setting ( $9 \%$ ) have been omitted due to modifications to the question and/or scenario to better fit the setting, thus limiting comparability across inpatient and ambulatory care settings. 
other services do not provide consultative services on their patients). ${ }^{a}$ This finding varied by level of training and specialty grouping (see Appendix B22).

Nearly $83 \%$ of the faculty members in the group interviews indicated that they had an objective way of knowing which procedures a particular resident or fellow was allowed to perform without direct supervision. ${ }^{a}$ In many CLEs, faculty members and program directors reported that this information is documented in an online system and maintained by GME or individual programs.

Generally, faculty members and programs directors indicated that nurses and other clinical staff members had mechanisms (eg, paper or online methods) to verify the level of supervision needed when residents and fellows perform clinical procedures outside of the operative areas. During the walking rounds, it appeared that the nurses had limited access to this information, varied in their awareness of how to access this information, or did not routinely use this information in the course of clinical care. Often, this information appeared to be incomplete or inaccurate. In nearly all CLEs (98.0\%), nurses reported that, in the absence of an attending physician, they relied principally on familiarity, trust, or year of training when residents and fellows performed procedures (Figure 13; see also Appendix C8).

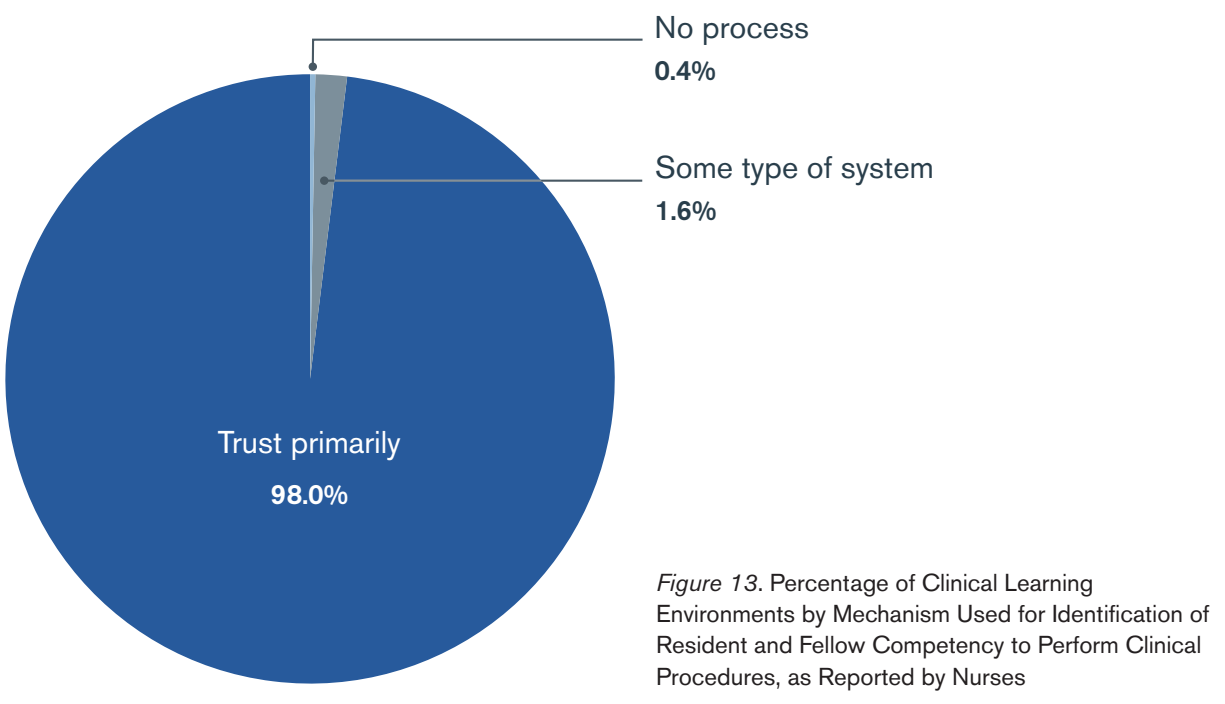

\section{Patient Safety Events Related to Supervision}

In $19.0 \%$ of the CLEs, the patient safety and quality leaders recalled patient safety events in the past year related to resident and fellow supervision. In the group interviews, $25.1 \%$ of program directors reported that, in the past year, they had to manage an issue of resident or fellow supervision that involved a patient safety event. Executive leaders were often unaware of patient safety events attributed to supervision. Generally across CLEs, the physician and leadership groups varied in their awareness of patient safety events related to resident and fellow supervision.

In general, executive and patient safety and quality leaders indicated that they addressed patient safety events as they arose and through retrospective review of the events. It was uncommon for CLEs to actively monitor for potential patient safety events related to supervision; the issue of supervision was often viewed as the responsibility of the GME community.

\footnotetext{
${ }^{\text {a }}$ Results from visits that were held exclusively in the ambulatory care setting ( $9 \%$ ) have been omitted due to modifications to the question and/or scenario to better fit the setting, thus limiting comparability across inpatient and ambulatory care settings.
} 


\section{FATIGUE MANAGEMENT, MITIGATION, AND DUTY HOURS}

In the area of fatigue management, mitigation, and duty hours, the CLER Program explored the culture of reporting duty hours, fatigue management, patient safety events related to fatigue, situations that increase the risk for burnout, and strategies to address fatigue and burnout.

\section{Culture of Reporting Duty Hours}

In the group interviews, the CLER Site Visit teams presented the residents and fellows with a scenario in which a colleague stays 30 minutes beyond his or her duty-hour limits to address a small, nonurgent clinical task. When asked about the likelihood that their colleague would report the time, 37.3\% responded that it was very unlikely that their colleague would do so. When presented with the same scenario, $20.8 \%$ of the faculty members and $12.3 \%$ of the program directors indicated that it was very unlikely that the resident or fellow would report the time.

In a separate query, $29.4 \%$ of the program directors in the group interviews indicated that when moonlighting was permitted, residents and fellows may be underreporting their moonlighting hours (median [IQR], 25.0\% [0\%-100\%] across CLEs).

\section{Fatigue Management}

At each of the site visits, the residents and fellows in the group interviews were asked to consider a hypothetical scenario in which they were maximally fatigued yet had 2 hours left before the end of their shift or work day. In this circumstance, $33.0 \%$ of the residents and fellows reported that they would power through to handoff or the end of the day. Responses varied by gender, year of training, and specialty grouping. Across CLEs, the median (IQR) finding was $27.9 \%$ (11.1\%-50.0\%); responses varied by region, type of ownership, and CLE bed size (Figure 14; see also Appendix B23).

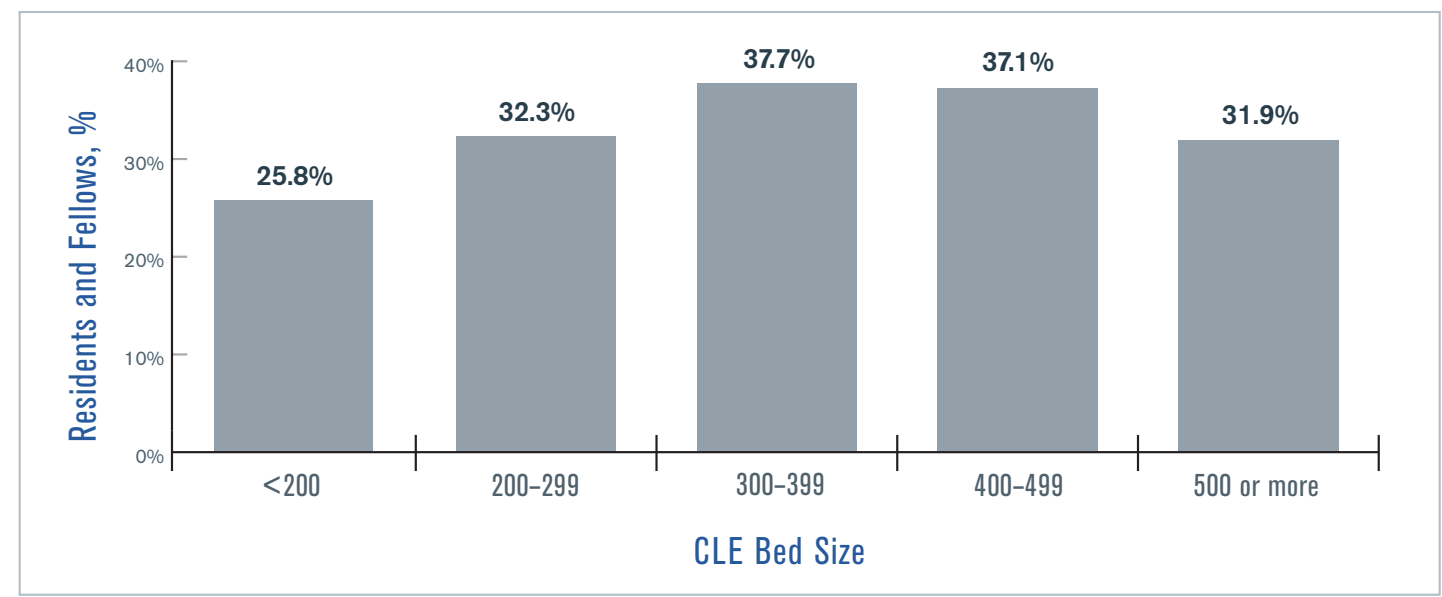

Figure 14. Percentage of Residents and Fellows Who Reported They Would Power Through When Maximally Fatigued, by Clinical Learning Environment (CLE) Bed Size

In this same circumstance, $36.0 \%$ indicated that they would notify a supervisor and expect to be taken off duty immediately; $14.6 \%$ indicated that they would ask another resident to take over their responsibilities.

When presented with the same scenario, $18.7 \%$ of the faculty members and $15.3 \%$ of the program directors in the group interviews expressed the belief that the resident or fellow would power through to handoff or to the end 
of the day. Approximately $54 \%$ of the faculty members and $56.6 \%$ of the program directors believed that the resident or fellow would notify his or her supervisor and expect to be taken off duty immediately.

The CLER Site Visit teams also explored issues of fatigue among faculty members and program directors by presenting a scenario in which their department, practice, or ambulatory care setting is short staffed and one of their colleagues is fatigued but scheduled for call that evening. In response to the scenario, $24.4 \%$ of the faculty members and $15.4 \%$ of the program directors indicated that the colleague would continue with his or her clinical schedule, including call.

In describing the situations that increase the risk for fatigue, the physician groups often mentioned the following: time spent on electronic health records, times of high patient volume and acuity, covering multiple hospitals, 24-hour shifts, telephone calls with multiple interruptions, moving from day to night shifts, telephone calls for nonurgent problems, a full day of clinical work after home call, and completing other clinical and documentation tasks during off duty hours.

\section{Patient Safety Events Related to Fatigue}

When queried in the physician interviews, $6.1 \%$ of the residents and fellows, $3.5 \%$ of the faculty members, and $2.4 \%$ of the program directors recalled a patient safety event related to resident or fellow fatigue (Figure 15). The CLER Site Visit teams also asked the patient safety and quality leaders a similar question. In $1.9 \%$ of the CLEs, the patient safety and quality leaders recalled 1 or more patient safety events related to resident or fellow fatigue (see Appendix C9). Occasionally, program directors were aware of patient safety events related to resident or fellow fatigue that appeared to be unknown to the patient safety and quality leaders. Mechanisms to assess risks to patient safety due to resident and fellow workload intensity was uncommon in many CLEs.

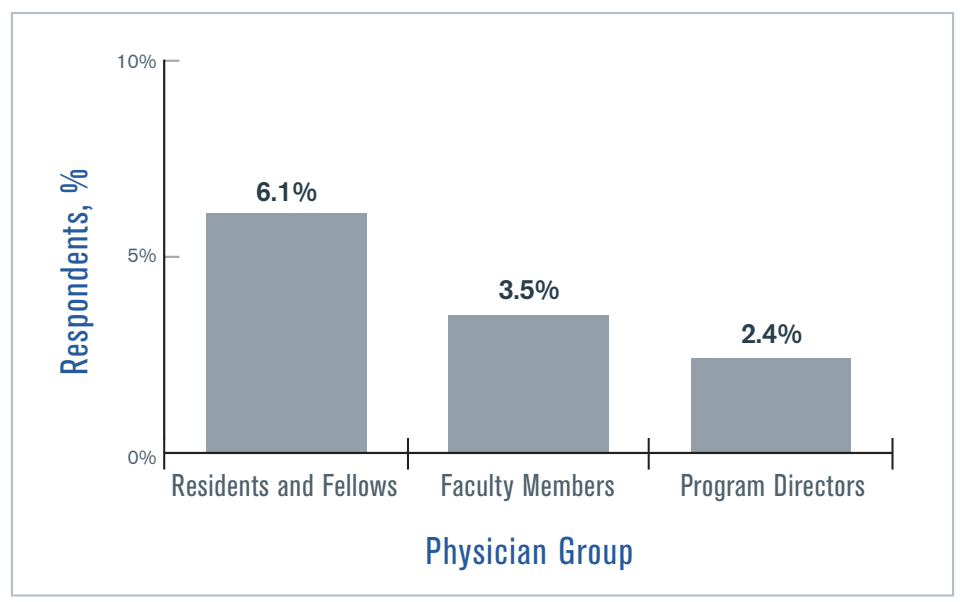

Figure 15. Percentage of Physicians Who Reported Awareness of a Patient Safety Event Related to Resident or Fellow Fatigue, by Physician Group 


\section{Situations That Increase the Risk for Burnout}

In many CLEs, residents and fellows described seeing signs of burnout in their colleagues that included emotional exhaustion, depersonalization, and/or a sense of low personal accomplishment. Residents and fellows often identified high patient volume, patient acuity, and nonphysician responsibilities as contributing factors to burnout.

In $50.6 \%$ of the CLEs, residents and fellows reported observing some signs of burnout among their faculty members and program directors (Figure 16; see also Appendix C10). Some of the manifestations included withdrawal from others and lack of willingness or enthusiasm to teach.

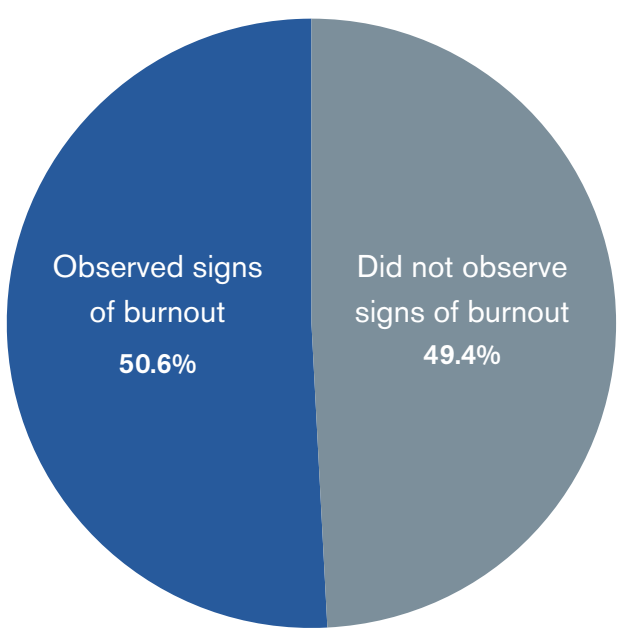

Figure 16. Percentage of Clinical Learning Environments Where Residents and Fellows Observed Some Signs of Burnout Among Faculty Members and Program Directors

When asked about burnout, faculty members and program directors mentioned the same factors identified by the residents and fellows and added clinical productivity pressures, extensive documentation requirements, inadequate clinical and administrative support, and the challenge of balancing teaching, research, administrative responsibilities, and patient care.

Many residents, fellows, faculty members, and program directors also perceived that connection to community and the small size of the institution contributed to enhanced collegiality and a greater sense of professional acknowledgment that helped mitigate possible burnout among faculty members and program directors.

\section{Strategies to Address Fatigue and Burnout}

In general, CLE efforts to mitigate fatigue focused primarily on provision of sleep rooms, transportation home for residents and fellows when they were too tired to drive, backup call systems, and education on fatigue management and mitigation.

Systematic strategies to identify, mitigate, and prevent fatigue and burnout were uncommon across CLEs. When strategies existed, they were often in response to an event related to fatigue or burnout. The content and coordination of these efforts varied across CLEs, and measures to assess the effectiveness of these efforts were uncommon. 


\section{PROFESSIONALISM}

The concept of professionalism encompasses a number of attributes. The CLER Site Visits focused mainly on those involving honesty, integrity, and respectful treatment of others.

During each visit, the CLER Site Visitors asked executive leaders whether or not any GME-related incidents involving professionalism or integrity had occurred in the past year. The executive leaders in $44.4 \%$ of the CLEs indicated that 1 or more such incidents had been brought to their attention (see Appendix C11).

\section{Honesty in Reporting}

Across CLEs, a median (IQR) of 100\% (85.7\%-100\%) of the residents and fellows in the group interviews reported that they believe their CLE provides a supportive, nonpunitive environment for bringing forward concerns about honesty in reporting. This finding varied by region and type of ownership (see Appendix B24).

Overall, $23.6 \%$ of the residents and fellows in the group interviews reported that while at their CLE, they had documented a history or physical finding in a patient medical record that they did not personally elicit (eg, copying and pasting from another note without attribution). Appendix B25 provides detailed information on variability. Approximately $5 \%$ of the faculty members and $5.5 \%$ of the program directors reported that they believed that a majority of residents and fellows had engaged in this practice.

When the CLER Site Visit teams asked the faculty members about their documentation practices, $9.8 \%$ in the group interviews indicated that they had documented a history or physical finding in a patient medical record that they did not personally elicit.

Occasionally, the executive leadership indicated that, in the past year, they had identified professionalism issues related to documentation practices using electronic health records and other sources of protected health information.

\section{Integrity}

Of the residents and fellows surveyed in the group interviews, $11.1 \%$ reported that while at the CLE, they had on occasion felt pressured to compromise their honesty or integrity to satisfy an authority figure (see Appendix B26 for detailed information on variability).

To further explore issues of integrity, the CLER Site Visit teams presented the residents and fellows in the group interviews with a scenario in which 1 of their colleagues has written a manuscript and the department chair or program director-although not involved in the project-asked to be included as an author. Approximately $59 \%$ of the residents and fellows responded that they would advise the colleague to discuss the matter with a faculty member or their designated institutional official. The next common response was to advise their colleague to speak with the chief medical officer.

\section{Respectful Treatment of Others}

Generally across CLEs, the executive leadership expressed intolerance for unprofessional and disrespectful behavior. Nearly $90 \%$ of the program directors surveyed expressed the belief that their CLE was usually or always effective in managing reports of unprofessional behavior. 
In the group discussions, the CLER Site Visit teams presented the residents and fellows with a scenario describing an attending physician's mistreatment of a resident colleague that continued to persist despite being reported to the chief resident, program director, department chair, and head of GME. When presented with choices of what they might advise the colleague to do, most (59.6\%) indicated that they would recommend contacting the organization's human resources department or anonymous hotline. Other responses included: contacting the Equal Employment Opportunity Commission (8.1\%), registering a concern with the ACGME (16.1\%), and taking no further action (2.3\%). Another 13.9\% suggested they would advise some other course of action, and when asked to elaborate, many indicated that they would recommend speaking with the ombudsman or going back to 1 or more members of the GME community to seek assistance.

When presented with the same scenario, faculty members and program directors in the group interviews also varied in their belief as to what the resident would do to address the perceived mistreatment.

Although many residents, fellows, nurses, and other health care professionals described their work environments as respectful and collegial, in nearly all of the CLEs (83.9\%), individuals across multiple areas described the behavior of attending physicians and nurses as disrespectful or disruptive. In $53.6 \%$ of the CLEs, the behaviors were described as chronic, persistent, or pervasive in nature (Figure 17; see also Appendix C12).

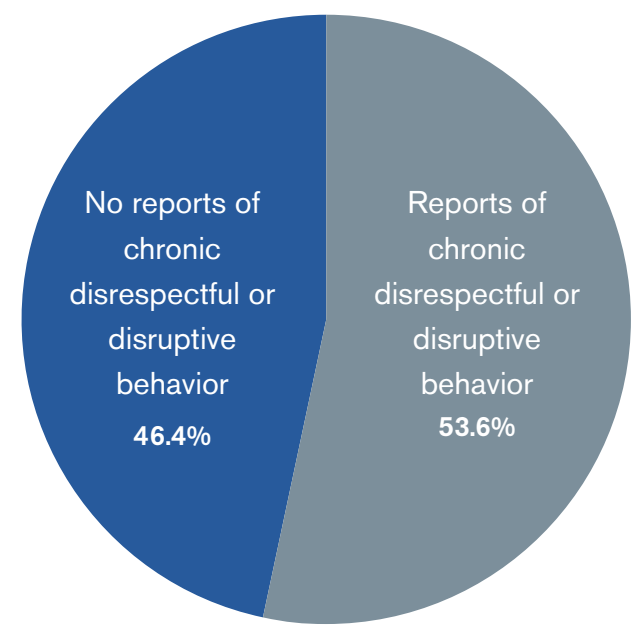

Figure 17. Percentage of Clinical Learning Environments Where Chronic Disrespectful or Disruptive Behavior Was Reported Across More Than 1 Clinical Unit

Across CLEs, many residents and fellows also described professionalism issues in obtaining consultation services, including lack of responsiveness and disrespectful communication in response to their requests for consultation.

Generally across CLEs, residents and fellows appeared to be aware of the mechanisms and resources available to resolve perceived mistreatment beyond those offered by GME. The effectiveness of the organization's response to address these concerns varied across CLEs. On occasion, residents, fellows, and other clinical staff mentioned that they would not report mistreatment out of concern for adverse consequences of reporting. 


\section{REFERENCE}

1. Weiss KB, Bagian JP, Wagner R. CLER Pathways to Excellence: expectations for an optimal clinical learning environment [executive summary]. J Grad Med Educ. 2014;6(3):610-611.

\section{${ }^{*}$ CLER PROGRAM}

Mark R. Bixby, MD, FAAFP; Isabelle Bourgeois, MPA; Jennifer J. Buescher, MD, MSPH; Robert Casanova, MD, MHPE;

Baretta R. Casey, MD, MPH, FAAFP**; Marian D. Damewood, MD, FACOG; Kevin C. Dellsperger, MD, PhD; Robin Dibner, MD;

Staci A. Fischer, MD; Paula Hensley, MPH; John A. Hopper, MD; Sharhabeel Jwayyed, MD, MS; Catherine Kallal, MD**;

Elizabeth Kimball, MA**; Nancy J. Koh, PhD; Kathryn E. McGoldrick, MD, MAH, FCAl(Hon); Clifton McReynolds, PhD;

Terrie Mendelson, MD**; Joshua Mirôn, MA; Robin C. Newton, MD, FACP; Daniel Picard, MD**; Kathy B. Porter, MD, MBA, FACOG;

Dale Ray, MD, MMM; Laura Riordan, MS; Melissa Schori, MD, FACP, MBA; Stephen Smith, MD**; Mike Strickland, MFA*;

Hongling Sun, PhD; Marie Trontell, MD; Paul Uhlig, MD, MPA; Robin Wagner, RN, MHSA; Elizabeth Wedemeyer, MD;

Kevin B. Weiss, MD; Esther Woods; Martha S. Wright, MD, MEd; James R. Zaidan, MD, MBA; Jose Zayas, DO, FAAP

**Former staff member 


\section{Lessons Learned and Future Directions}

Robin Wagner, RN, MHSA, and Kevin B. Weiss, MD

\section{LESSONS LEARNED}

The Clinical Learning Environment Review (CLER) Program is a formative evaluative experience for the nearly 800 Accreditation Council for Graduate Medical Education (ACGME) institutions that sponsor graduate medical education (GME) programs. Through regular site visits, the CLER Program assesses how the clinical learning environments (CLEs) of these Sponsoring Institutions (SIs) are performing in 6 focus areas of importance to the ACGME. ${ }^{1}$ CLER's 2016 and 2018 national reports ${ }^{2,3}$ included findings from the first 2 sets of CLER Site Visits, which were conducted at larger SIs (ie, those with 3 or more core residency programs). The present CLER National Report of Findings 2019 includes findings for baseline visits to smaller Sls (ie, those with 2 or fewer core residency programs).

This report offers, for the first time, a look at almost all of the Sls that were not covered in the first 2 CLER national reports. (CLER will publish a special report on the smallest SIs [ie, preventive medicine programs and single fellowship programs] in late 2019.) These 2 separate samples (ie, larger and smaller Sls) were created because of an operational need, as well as knowledge that, due to their unique features, the smaller Sls would require an adapted protocol. Beginning with Protocol 3.0 (see "Future Directions"), the CLER Program began visiting both smaller and larger Sls during a single cycle.

Of note, the CLER Program has chosen not to directly compare these 2 samples, as the intention of this separate set of visits was simply to extend CLER's initial findings. A detailed analysis of some CLE characteristics may still shed light on differences not determined by size of the program. CLER Program staff hope that information will be forthcoming.

This CLER National Report of the smaller Sls highlights the important role that ambulatory care environments play in the resident and fellow experience. As with the report of the larger SIs, it also reveals a large degree of variability both within and across CLEs in each of the 6 CLER Focus Areas. This variability can be the result of CLE efforts to bring about positive change. It can also be a sign of CLE processes that are inefficient or ineffective, thereby representing opportunities for improvement.

\section{FUTURE DIRECTIONS}

CLER Protocol 3.0, which will inform the findings for the next CLER national report, was launched in June 2017 in the first single combined cycle for all ACGME-accredited Sls. This cycle, which is currently targeted for completion in fall 2019, will include site visits to well over 700 Sls. The CLER Program will continue to visit all ACGME-accredited SIs on a recurring basis approximately every $24( \pm 6)$ months and will continue to visit 1 participating site per $\mathrm{SI}$.

Going forward, the CLER Program will continue to explore interprofessional learning and collaborative practice in CLEs. The CLER Site Visits identified that, often, nurses, residents, and fellows work in parallel rather than in an integrated fashion. As noted in overarching theme 4 (pp 26-27), CLEs were found to have a lack of interprofessional collaborative educational or learning experiences. Based on these findings, the CLER 
Program will be examining how the concept of "teaming" can highlight important attributes of a high-performing learning health system and CLE. The CLER Program anticipates that, over time, it will deepen its exploration of how CLEs invest in, deliberately design, and monitor new models to promote learning and performance within clinical care teams-thereby strengthening the association between the quality of the GME experience and the quality of patient care in general.

To date, the CLER Site Visit findings shine an important light on how residents and fellows learn in the context of delivering patient care in extremely busy health care systems. These findings indicate the need to better understand attributes of high-performing CLEs that may be associated with health systems seeking to define themselves as high-performing learning health systems. The efforts of the National Academy of Medicine and other related work in the areas of learning health systems and high-reliability organizations ${ }^{4.5}$ indicate that GME will likely benefit from CLEs who have explicitly focused their organizational efforts on operationalizing and sustaining these concepts.

Built on a model of quality improvement, the CLER Program will continue to explore new opportunities to provide the nation's CLEs with information they can use to simultaneously optimize learning and patient care. One mechanism for doing so has been the introduction of subprotocols to enhance the regular site visit process. The first of these subprotocols focuses on the operative and procedural areas; a second subprotocol will provide insights from the patient perspective. In the future, the CLER Program will also explore the perspective of governance and governing bodies' understanding of the mission and goals of their CLEs-particularly as they relate to GME. The CLER Program will also seek to deepen understanding of the structure and function of medical education across the medical continuum, specifically lifelong learning as seen through continuing professional development.

As a final note, the CLER Program would not be possible without the efforts of a large supportive community. The authors of this report thank many members of that community including: the ACGME Board of Directors for its continued interest in the ongoing development of this program; the CLER Program staff; the members of the CLER Evaluation Committee; other programs within the ACGME who have helped support CLER; and most importantly, all of the individuals within the nation's SIs and CLEs who have helped organize and participate in the CLER visits.

\section{REFERENCES}

1. Weiss KB, Bagian JP, Wagner R. CLER Pathways to Excellence: expectations for an optimal clinical learning environment [executive summary]. J Grad Med Educ. 2014;6(3):610-611. doi:10.4300/JGME-D-14-00348.1

2. Wagner R, Koh NJ, Patow C, et al. Detailed findings from the CLER National Report of Findings 2016. J Grad Med Educ. 2016;8(2s1):35-54. doi:10.4300/1949-8349.8.2s1.35

3. Koh NJ, Wagner R, Newton R, et al. Detailed findings from the CLER National Report of Findings 2018. J Grad Med Educ. 2018;10(4s):49-68. doi:10.4300/1949-8349.8.2s1.35

4. Institute of Medicine. The Learning Healthcare System: Workshop Summary. Washington, DC: The National Academies Press; 2007. doi:10.17226/11903

5. Weick KE, Sutcliffe KM. Organizing for reliability: processes of collective mindfulness. In: Sutton RS SB, ed. Research in Organizational Behavior. Stamford, CT: Jai Pres; 1999:81-123. 


\section{Appendix A1. \\ GENERAL CHARACTERISTICS OF SPONSORING INSTITUTIONS}

A1.1. Sponsoring Institution Distribution by Region and Type ${ }^{a}$

\begin{tabular}{|l|c|c|}
\hline Characteristic & $\begin{array}{c}\text { SIs With CLER Visits, \% } \\
(\mathbf{n = 2 7 0 )}\end{array}$ & $\begin{array}{c}\text { All SIs, \% } \\
(\mathbf{N}=\mathbf{7 2 5})\end{array}$ \\
\hline Region & & 24.0 \\
\hline Northeast & 23.7 & 22.9 \\
\hline Midwest & 23.0 & 30.9 \\
\hline South & 28.1 & 20.6 \\
\hline West & 22.2 & 1.7 \\
\hline Territory & 3.0 & \\
\hline Type of SI & & 55.3 \\
\hline General/teaching hospital & 70.4 & 14.9 \\
\hline Medical school or health science center & 5.2 & 3.9 \\
\hline Educational consortium & 3.7 & 2.5 \\
\hline Children's hospital & 0.7 & 23.4 \\
\hline Other & 20.0 & \\
\hline
\end{tabular}

A1.2. Sponsoring Institution Distribution by Number of ACGME-Accredited Residency and Fellowship Programs and Participating Sites ${ }^{a}$

\begin{tabular}{|l|c|c|}
\hline Programs and Sites, No. & $\begin{array}{c}\text { Sls With CLER Visits, \% } \\
(\mathbf{n = 2 7 0 )}\end{array}$ & $\begin{array}{c}\text { All SIs, \% } \\
(\mathbf{N}=\mathbf{7 2 5})\end{array}$ \\
\hline Programs & & 36.0 \\
\hline 1 & 49.6 & 12.1 \\
\hline 2 & 25.9 & 51.9 \\
\hline$\geq 3$ & 24.4 & \\
\hline Core Programs & & 10.5 \\
\hline 0 & 0.7 & 33.9 \\
\hline 1 & 67.0 & 55.6 \\
\hline$\geq 2$ & 32.2 & 16.0 \\
\hline Participating Sites & & 15.6 \\
\hline$<3$ & 25.6 & 21.1 \\
\hline $3-4$ & 25.6 & 47.3 \\
\hline $5-9$ & 27.0 & \\
\hline$>9$ & 21.9 & \\
\hline
\end{tabular}

a Percentages do not total 100 because of rounding.

b Limited to 8 Sponsoring Institutions (SIs) in Puerto Rico.

Abbreviations: ACGME, Accreditation Council for Graduate Medical Education; CLER, Clinical Learning Environment Review. 
A 1.3. Number and Distribution of Core Faculty Members at Sponsoring Institutions by Specialty Grouping ${ }^{2}$

\begin{tabular}{|l|c|c|}
\hline Specialty Subgroup & $\begin{array}{c}\text { SIs With CLER Visits, \% } \\
(\mathbf{n = 3 6 8 9 )}\end{array}$ & $\begin{array}{c}\text { All SIs, \% } \\
(\mathbf{N}=62,916)\end{array}$ \\
\hline Medical & 76.7 & 36.0 \\
\hline Surgical & 10.9 & 36.0 \\
\hline Hospital-based & 12.4 & 28.0 \\
\hline
\end{tabular}

\section{A 1.4. Number and Distribution of Residents and Fellows at Sponsoring Institutions by Gender, Level of Training, and Specialty Groupinga}

\begin{tabular}{|c|c|c|}
\hline Resident and Fellow Characteristic & $\begin{array}{l}\text { SIs With CLER Visits, \% } \\
\qquad(\mathrm{n}=9818)\end{array}$ & $\begin{array}{c}\text { All SIs, \% } \\
(\mathrm{N}=127,924)\end{array}$ \\
\hline \multicolumn{3}{|l|}{ Gender } \\
\hline Male & 50.8 & 52.9 \\
\hline Female & 47.8 & 44.4 \\
\hline Unknown & 1.5 & 2.7 \\
\hline \multicolumn{3}{|l|}{ Level of Training } \\
\hline PGY-1 & 36.9 & 36.7 \\
\hline PGY-2 & 30.7 & 27.9 \\
\hline PGY-3 & 29.2 & 25.1 \\
\hline PGY-4+ & 3.2 & 10.3 \\
\hline \multicolumn{3}{|l|}{ Specialty Group } \\
\hline Medical & 88.6 & 59.9 \\
\hline Surgical & 5.9 & 20.4 \\
\hline Hospital-based & 5.4 & 19.6 \\
\hline
\end{tabular}




\section{Appendix A2. \\ GENERAL CHARACTERISTICS OF CLINICAL LEARNING ENVIRONMENTS}

\section{A2.1. Clinical Learning Environment Distribution by Type of Ownership and Services ${ }^{a, b}$}

\begin{tabular}{|c|c|c|c|}
\hline Characteristic & $\begin{array}{l}\text { Sls With CLER Visits, \% } \\
\qquad(n=251)^{c}\end{array}$ & $\begin{array}{l}\text { Teaching Hospitals, }{ }^{d} \% \\
(n=314)\end{array}$ & $\begin{array}{l}\text { All Hospitals, } \% \\
(\mathrm{~N}=6251)\end{array}$ \\
\hline \multicolumn{4}{|l|}{ Type of Ownership } \\
\hline Nongovernment, not-for-profit & 70.9 & 65.9 & 49.6 \\
\hline Investor-owned, for-profit & 12.4 & 2.5 & 26.8 \\
\hline Government, federal & 5.2 & 15.0 & 3.5 \\
\hline Government, nonfederal & 11.6 & 16.6 & 20.1 \\
\hline \multicolumn{4}{|l|}{ Service for Majority of Patients } \\
\hline General medical and surgical & 94.0 & 91.1 & 74.7 \\
\hline Other ${ }^{\mathrm{e}}$ & 6.0 & 8.9 & 25.3 \\
\hline
\end{tabular}

\section{A2.2. Clinical Learning Environment Distribution by Beds and Staffing ${ }^{a}$}

\begin{tabular}{|l|c|c|c|}
\hline Characteristic & $\begin{array}{c}\text { CLER Program, } \\
\text { Median (IQR) }\end{array}$ & $\begin{array}{c}\text { Teaching Hospitals, } \\
\text { Median (IQR) }\end{array}$ & $\begin{array}{c}\text { All Hospitals, } \\
\text { Median (IQR) }\end{array}$ \\
\hline Total Licensed Beds & $387(253-523)^{\dagger}$ & $600(402-789)^{g}$ & $102(40-253)^{\mathrm{h}}$ \\
\hline Total Staffed Beds & $295(183-424)$ & $498(319-682)$ & $81(32-198)$ \\
\hline Staffi, & & & \\
\hline \multicolumn{1}{|c|}{ Registered nurses } & $629(391-932)$ & $1371(746-2121)$ & $104(46-346)$ \\
\hline Clinical staff & $374(243-559)$ & $733(435-1256)$ & $96(46-233)$ \\
\hline All other personnel & $1012(594-1446)$ & $2095(1277-3203)$ & $205(91-550)$ \\
\hline
\end{tabular}

\footnotetext{
a Based on the 2015 American Hospital Association Annual Survey.

b Percentages do not total 100 because of rounding.

${ }^{c}$ Missing data $(<8 \%)$ largely due to clinical sites that do not report data to the American Hospital Association. Percentages based on valid percent.

${ }^{d}$ Member of Council of Teaching Hospital of the Association of American Medical Colleges.

e Includes psychiatric, rehabilitation, acute long-term care hospital, children's general medical and surgical, and other types of services.

${ }^{\mathrm{f}}$ Missing data $<20 \%$.

g Missing data $<14 \%$.

${ }^{\mathrm{h}}$ Missing data $<25 \%$.

Physicians, residents, interns, and other trainees omitted from staff count.

Full-time and part-time personnel only; excludes full-time and part-time equivalent personnel.

Abbreviations: CLER, Clinical Learning Environment Review; IQR, interquartile range; SI, Sponsoring Institution.
} 


\section{Appendix A3. \\ CLINICAL LEARNING ENVIRONMENTS VISITED: \\ NUMBER OF PROGRAMS AT SITE}

\begin{tabular}{|l|c|}
\hline Programs at Site, No., ${ }^{a, b}$ & $\begin{array}{c}\text { SIs With CLER Visits, \% } \\
(\mathbf{N}=\mathbf{2 7 0})\end{array}$ \\
\hline Programs & \\
\hline 1 & 48.9 \\
\hline 2 & 24.8 \\
\hline$\geq 3$ & 26.3 \\
\hline Core Programs & 1.5 \\
\hline 0 & 65.6 \\
\hline 1 & 33.0 \\
\hline$\geq 2$
\end{tabular}

a Based on the 2017 Accreditation Council for Graduate Medical Education data

${ }^{b}$ Percentages do not total 100 because of rounding.

Abbreviations: CLER, Clinical Learning Environment Review; SI, Sponsoring Institution. 


\section{Appendix A4. \\ CLINICAL LEARNING ENVIRONMENTS VISITED: CHARACTERISTICS OF GROUPS INTERVIEWED}

A4.1. Selected Characteristics of Residents and Fellows in the Group Interviews ${ }^{a}$

\begin{tabular}{|l|c|}
\hline Characteristic & $\begin{array}{c}\text { Residents and Fellows, \% } \\
\text { (N=1840) }\end{array}$ \\
\hline \begin{tabular}{|l|} 
Gender \\
Male
\end{tabular} & 51.7 \\
\hline Female & 48.3 \\
\hline Level of Training & \\
\hline PGY-1 & 3.5 \\
\hline PGY-2 & 44.6 \\
\hline PGY-3 & 40.7 \\
\hline PGY-4+ & 11.2 \\
\hline Specialty Group ${ }^{b}$ & \\
\hline Medical & 86.8 \\
\hline Surgical & 6.5 \\
\hline Hospital-based & 6.7 \\
\hline
\end{tabular}

A4.2. Selected Characteristics of Faculty Members and Program Directors in the Group Interviews ${ }^{a, c}$

\begin{tabular}{|c|c|c|}
\hline Characteristic & $\begin{array}{l}\text { Faculty Members, } \% \text { d } \\
\qquad(N=1789)\end{array}$ & $\begin{array}{l}\text { Program Directors, \% } \\
\qquad(\mathrm{N}=459)\end{array}$ \\
\hline \multicolumn{3}{|c|}{ Years at Hospital or Medical Center } \\
\hline$\leq 2$ & 20.5 & 5.7 \\
\hline $3-5$ & 21.4 & 15.0 \\
\hline $6-10$ & 19.6 & 23.2 \\
\hline$>10$ & 37.3 & 56.1 \\
\hline \multicolumn{3}{|l|}{ Program ${ }^{b}$} \\
\hline Core residency program & 87.5 & 74.7 \\
\hline Fellowship & 3.9 & 22.5 \\
\hline Both & 8.6 & 2.8 \\
\hline \multicolumn{3}{|l|}{ Specialty Group ${ }^{b}$} \\
\hline Medical & 82.5 & 81.4 \\
\hline Surgical & 7.6 & 10.5 \\
\hline Hospital-based & 9.9 & 8.1 \\
\hline
\end{tabular}

\footnotetext{
a Based on audience response system data.

${ }^{\mathrm{b}}$ Missing data $(<2 \%)$ have been omitted; percentages based on valid percent.

c Percentages do not total 100 because of rounding.

${ }^{d}$ Of faculty members interviewed, $1.2 \%$ were nonteaching faculty.

Abbreviation: PGY, postgraduate year.
} 
$92 \mid$ APPENDIX A | CLER National Report of Findings 2019 


\section{Appendix B. \\ SELECTED RESULTS FROM RESIDENT AND FELLOW GROUP INTERVIEWS}

\section{B1. Percentage of Residents and Fellows Who Reported Experiencing an Adverse Event, Near Miss/Close Call, or Unsafe Condition}

PERCENT OF TOTAL

SURVEYED ${ }^{a}(\mathrm{~N}=1836)$

\section{7}

\section{PERCENTAGE BY RESIDENT AND \\ FELLOW AND CLE CHARACTERISTICS}

\begin{tabular}{|c|c|}
\hline $\begin{array}{l}\text { Resident and Fellow } \\
\text { Characteristics }^{\mathrm{a}}\end{array}$ & $\begin{array}{c}\text { Residents and Fellows, } \\
\qquad \%(n=1836)\end{array}$ \\
\hline \multicolumn{2}{|l|}{ Gender } \\
\hline Male & 65.3 \\
\hline Female & 68.1 \\
\hline \multicolumn{2}{|l|}{ Level of Training ${ }^{* *}$} \\
\hline PGY-1 & 48.4 \\
\hline PGY-2 & 63.8 \\
\hline PGY-3 & 75.6 \\
\hline PGY-4+ & 51.5 \\
\hline \multicolumn{2}{|l|}{ Specialty Group*** } \\
\hline Medical & 68.1 \\
\hline Surgical & 61.3 \\
\hline Hospital-based & 52.8 \\
\hline \multicolumn{2}{|l|}{ CLE Characteristics $^{b}$} \\
\hline \multicolumn{2}{|l|}{ Regionc } \\
\hline Northeast & 64.5 \\
\hline Midwest & 71.0 \\
\hline South & 69.3 \\
\hline West & 68.1 \\
\hline \multicolumn{2}{|l|}{ Bed Size } \\
\hline$<200$ & 67.8 \\
\hline $200-299$ & 64.1 \\
\hline 300-399 & 71.2 \\
\hline $400-499$ & 64.5 \\
\hline 500 or more & 71.1 \\
\hline \multicolumn{2}{|l|}{ Type of Ownership* } \\
\hline Nongovernment, not-for-profit & 68.2 \\
\hline Investor-owned, for-profit & 63.4 \\
\hline Government, federal & 70.4 \\
\hline Government, nonfederal & 69.2 \\
\hline
\end{tabular}

DISTRIBUTION ACROSS CLEs ${ }^{d}(n=268)$

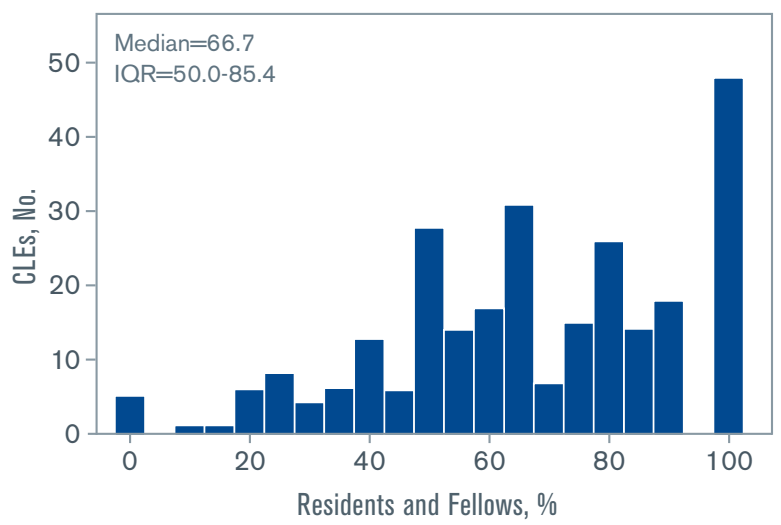

${ }^{a}$ Missing data $(<1 \%)$ have been omitted; percentages based on valid percent.

${ }^{\mathrm{b}}$ Missing data $(<7 \%)$ have been omitted; percentages based on valid percent. Of note, data are missing largely due to clinical sites that do not report data to the American Hospital Association.

${ }^{6}$ Results from clinical learning environments (CLEs) in Puerto Rico $(3 \%)$ omitted to ensure anonymity.

${ }^{d}$ Distribution includes $95 \%$ or more of the total number of CLEs $(\mathrm{N}=270)$.

* Statistically significant at $P<.05$.

** Statistically significant at $P<.01$.

*** Statistically significant at $P<.001$.

Abbreviation: PGY, postgraduate year. 
B2. Percentage of Residents and Fellows Who Reported Experiencing an Adverse Event, Near Miss/Close Call, or Unsafe Condition and Submitted a Report Through the Clinical Site's Reporting System

PERCENT OF TOTAL

SURVEYEDa $(\mathrm{N}=1195)$

\section{5}

\section{PERCENTAGE BY RESIDENT AND \\ FELLOW AND CLE CHARACTERISTICS}

\begin{tabular}{|c|c|}
\hline $\begin{array}{l}\text { Resident and Fellow } \\
\text { Characteristics }^{\mathrm{a}}\end{array}$ & $\begin{array}{c}\text { Residents and Fellows, } \\
\%(n=1195)\end{array}$ \\
\hline \multicolumn{2}{|l|}{ Gender* } \\
\hline Male & 60.1 \\
\hline Female & 52.8 \\
\hline \multicolumn{2}{|l|}{ Level of Training* } \\
\hline PGY-1 & 54.8 \\
\hline PGY-2 & 53.2 \\
\hline PGY-3 & 60.6 \\
\hline PGY-4+ & 50.5 \\
\hline \multicolumn{2}{|l|}{ Specialty Group } \\
\hline Medical & 56.8 \\
\hline Surgical & 52.8 \\
\hline Hospital-based & 54.7 \\
\hline \multicolumn{2}{|l|}{ CLE Characteristics $^{b}$} \\
\hline \multicolumn{2}{|l|}{ Region $^{c}$} \\
\hline Northeast & 58.1 \\
\hline Midwest & 56.0 \\
\hline South & 52.1 \\
\hline West & 62.5 \\
\hline \multicolumn{2}{|l|}{ Bed Size ${ }^{* *}$} \\
\hline$<200$ & 64.9 \\
\hline $200-299$ & 59.9 \\
\hline 300-399 & 57.3 \\
\hline $400-499$ & 49.4 \\
\hline 500 or more & 47.5 \\
\hline \multicolumn{2}{|l|}{ Type of Ownership ${ }^{* * *}$} \\
\hline Nongovernment, not-for-profit & 54.1 \\
\hline Investor-owned, for-profit & 51.8 \\
\hline Government, federal & 81.8 \\
\hline Government, nonfederal & 70.0 \\
\hline
\end{tabular}

DISTRIBUTION ACROSS CLES ${ }^{d}(n=263)$

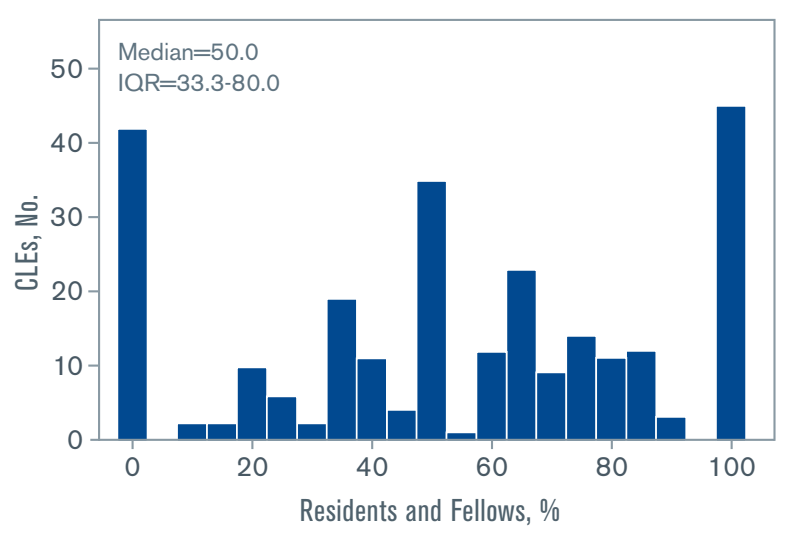

${ }^{a}$ Missing data $(<3 \%)$ have been omitted; percentages based on valid percent.

${ }^{\mathrm{b}}$ Missing data $(<7 \%)$ have been omitted; percentages based on valid percent. Of note, data are missing largely due to clinical sites that do not report data to the American Hospital Association.

${ }^{\mathrm{c}}$ Results from clinical learning environments (CLEs) in Puerto Rico (3\%) omitted to ensure anonymity.

${ }^{\mathrm{d}}$ Distribution includes $95 \%$ or more of the total number of CLEs $(\mathrm{N}=270)$.

* Statistically significant at $P<.05$.

** Statistically significant at $P<.01$.

*** Statistically significant at $P<.001$.

Abbreviation: PGY, postgraduate year. 


\section{B3. Percentage of Residents and Fellows Who Reported a Near Miss/Close Call Event}

\section{PERCENT OF TOTAL \\ SURVEYED ${ }^{a}(\mathrm{~N}=1838)$}

\section{5}

\section{PERCENTAGE BY RESIDENT AND \\ FELLOW AND CLE CHARACTERISTICS}

\begin{tabular}{|c|c|}
\hline $\begin{array}{l}\text { Resident and Fellow } \\
\text { Characteristics }^{\mathrm{a}}\end{array}$ & $\begin{array}{c}\text { Residents and Fellows, } \\
\%(n=1838)\end{array}$ \\
\hline \multicolumn{2}{|l|}{ Gender } \\
\hline Male & 28.6 \\
\hline Female & 30.7 \\
\hline \multicolumn{2}{|l|}{ Level of Training*** } \\
\hline PGY-1 & 21.9 \\
\hline PGY-2 & 28.2 \\
\hline PGY-3 & 34.3 \\
\hline PGY-4+ & 20.4 \\
\hline \multicolumn{2}{|l|}{ Specialty Group* } \\
\hline Medical & 30.7 \\
\hline Surgical & 23.5 \\
\hline Hospital-based & 22.0 \\
\hline \multicolumn{2}{|l|}{ CLE Characteristics $^{\mathrm{b}}$} \\
\hline \multicolumn{2}{|l|}{ Regionc } \\
\hline Northeast & 26.3 \\
\hline Midwest & 30.9 \\
\hline South & 33.6 \\
\hline West & 30.0 \\
\hline \multicolumn{2}{|l|}{ Bed Size* } \\
\hline$<200$ & 35.0 \\
\hline $200-299$ & 26.9 \\
\hline 300-399 & 32.8 \\
\hline $400-499$ & 27.0 \\
\hline 500 or more & 26.6 \\
\hline \multicolumn{2}{|l|}{ Type of Ownership* } \\
\hline Nongovernment, not-for-profit & 28.3 \\
\hline Investor-owned, for-profit & 36.2 \\
\hline Government, federal & 38.0 \\
\hline Government, nonfederal & 32.8 \\
\hline
\end{tabular}

DISTRIBUTION ACROSS CLES ${ }^{d}(n=268)$

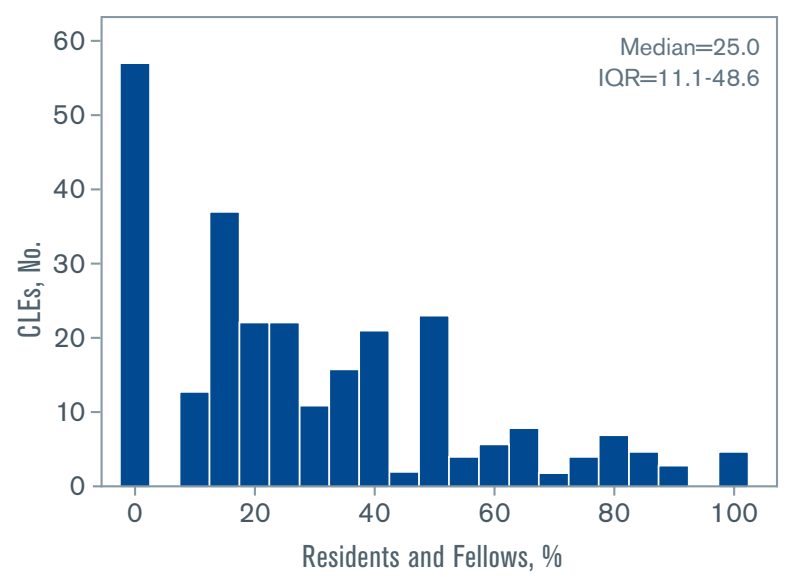

${ }^{a}$ Missing data $(<1 \%)$ have been omitted; percentages based on valid percent.

${ }^{\mathrm{b}}$ Missing data $(<7 \%)$ have been omitted; percentages based on valid percent. Of note, data are missing largely due to clinical sites that do not report data to the American Hospital Association.

${ }^{\circ}$ Results from clinical learning environments (CLEs) in Puerto Rico (3\%) omitted to ensure anonymity.

${ }^{d}$ Distribution includes $95 \%$ or more of the total number of CLEs $(\mathrm{N}=270)$.

* Statistically significant at $P<.05$.

** Statistically significant at $P<.01$.

*** Statistically significant at $P<.001$.

Abbreviation: PGY, postgraduate year. 


\section{B4. Percentage of Residents and Fellows Who Reported Receiving Feedback on the Outcome of a Report Submitted ${ }^{a}$ Through the Clinical Site's Reporting System}

\section{PERCENT OF TOTAL}

SURVEYED ${ }^{\mathrm{b}}(\mathrm{N}=1048)$

\section{1}

\section{PERCENTAGE BY RESIDENT AND \\ FELLOW AND CLE CHARACTERISTICS}

\begin{tabular}{|l|l|}
\hline $\begin{array}{l}\text { Resident and Fellow } \\
\text { Characteristics }\end{array}$ & $\begin{array}{c}\text { Residents and Fellows, } \\
\text { \% }\end{array}$ \\
\hline $\begin{array}{l}\text { Gender* } \\
\text { Male }\end{array}$ \\
\hline Female \\
\hline Level of Training
\end{tabular}

DISTRIBUTION ACROSS CLEs ${ }^{e}(n=260)$

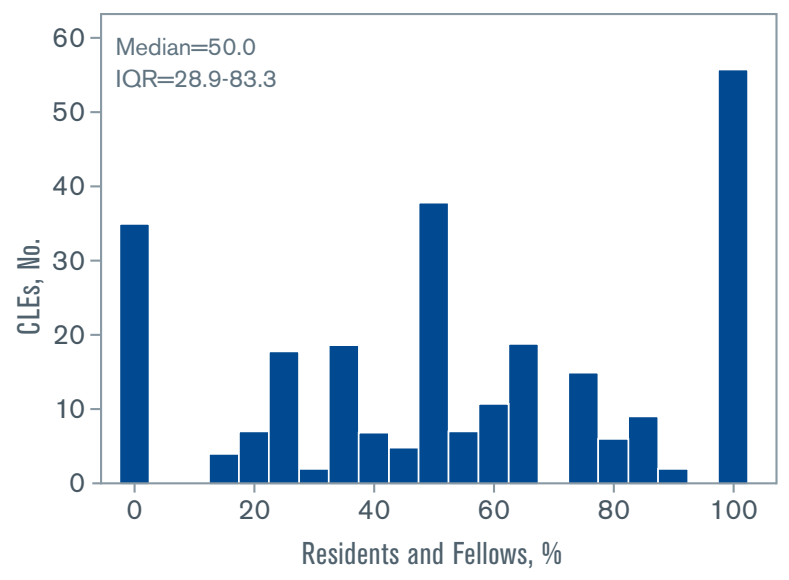

${ }^{\text {a }}$ Report submitted by resident or fellow or through a nurse, medical assistant, or supervisor.

${ }^{\mathrm{b}}$ Missing data $(<4 \%)$ have been omitted; percentages based on valid percent.

${ }^{\mathrm{c}}$ Missing data (<9\%) have been omitted; percentages based on valid percent. Of note, data are missing largely due to clinical sites that do not report data to the American Hospital Association.

${ }^{\mathrm{d}}$ Results from clinical learning environments (CLEs) in Puerto Rico (3\%) omitted to ensure anonymity.

${ }^{e}$ Distribution includes $95 \%$ or more of the total number of CLEs $(\mathrm{N}=270)$.

* Statistically significant at $P<.05$.

** Statistically significant at $P<.01$.

*** Statistically significant at $P<.001$.

Abbreviation: PGY, postgraduate year. 


\section{B5. Percentage of Residents and Fellows Who Reported Participating in an Interprofessional (Physicians, Nurses, Administrators, Others) Investigation of a Patient Safety Event (eg, Root Cause Analysis)}

\section{PERCENT OF TOTAL \\ SURVEYEDa $(\mathrm{N}=1838)$}

\section{8}

\section{PERCENTAGE BY RESIDENT AND \\ FELLOW AND CLE CHARACTERISTICS}

\begin{tabular}{|c|c|}
\hline $\begin{array}{l}\text { Resident and Fellow } \\
\text { Characteristics }^{\mathrm{a}}\end{array}$ & $\begin{array}{c}\text { Residents and Fellows, } \\
\qquad \%(\mathrm{n}=1838)\end{array}$ \\
\hline \multicolumn{2}{|l|}{ Gender } \\
\hline Male & 41.1 \\
\hline Female & 38.5 \\
\hline \multicolumn{2}{|l|}{ Level of Training*** } \\
\hline PGY-1 & 34.4 \\
\hline PGY-2 & 35.2 \\
\hline PGY-3 & 46.4 \\
\hline PGY-4+ & 36.4 \\
\hline \multicolumn{2}{|l|}{ Specialty Group } \\
\hline Medical & 40.9 \\
\hline Surgical & 34.5 \\
\hline Hospital-based & 31.7 \\
\hline \multicolumn{2}{|l|}{ CLE Characteristics $^{\text {b }}$} \\
\hline \multicolumn{2}{|l|}{ Region $^{c * * *}$} \\
\hline Northeast & 45.1 \\
\hline Midwest & 42.5 \\
\hline South & 34.4 \\
\hline West & 37.0 \\
\hline \multicolumn{2}{|l|}{ Bed Size* } \\
\hline$<200$ & 39.3 \\
\hline $200-299$ & 47.1 \\
\hline $300-399$ & 42.6 \\
\hline $400-499$ & 34.4 \\
\hline 500 or more & 37.6 \\
\hline \multicolumn{2}{|l|}{ Type of Ownership } \\
\hline Nongovernment, not-for-profit & 40.4 \\
\hline Investor-owned, for-profit & 37.9 \\
\hline Government, federal & 42.0 \\
\hline Government, nonfederal & 44.4 \\
\hline
\end{tabular}

DISTRIBUTION ACROSS CLES ${ }^{d}(n=268)$

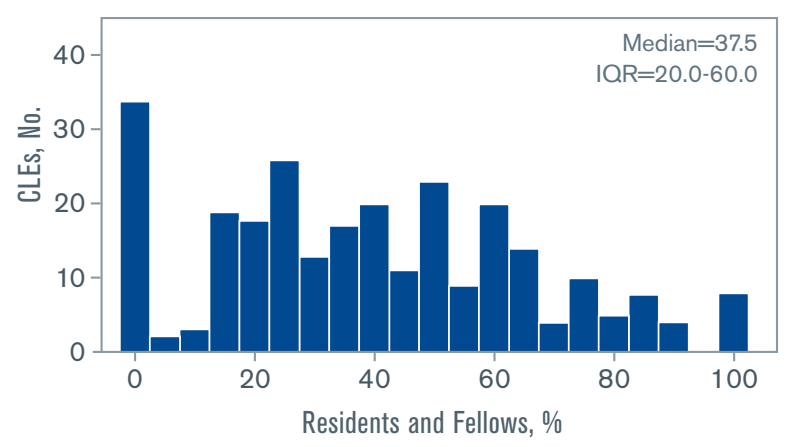

${ }^{a}$ Missing data $(<1 \%)$ have been omitted; percentages based on valid percent.

${ }^{\mathrm{b}}$ Missing data $(<7 \%)$ have been omitted; percentages based on valid percent. Of note, data are missing largely due to clinical sites that do not report data to the American Hospital Association.

${ }^{\circ}$ Results from clinical learning environments (CLEs) in Puerto Rico (3\%) omitted to ensure anonymity.

${ }^{d}$ Distribution includes $95 \%$ or more of the total number of CLEs $(\mathrm{N}=270)$.

* Statistically significant at $P<.05$.

** Statistically significant at $P<.01$.

*** Statistically significant at $P<.001$.

Abbreviation: PGY, postgraduate year. 
B6. Percentage of Residents and Fellows Who Reported Knowing the Clinical Site's Resources to Support Them if Involved in a Major Patient Safety Event

PERCENT OF TOTAL

SURVEYEDa $(\mathrm{N}=1840)$

\section{9}

\section{PERCENTAGE BY RESIDENT AND \\ FELLOW AND CLE CHARACTERISTICS}

\begin{tabular}{|c|c|}
\hline $\begin{array}{l}\text { Resident and Fellow } \\
\text { Characteristics }^{\mathrm{a}}\end{array}$ & $\begin{array}{l}\text { Residents and Fellows, } \\
\qquad \%(n=1840)\end{array}$ \\
\hline \multicolumn{2}{|l|}{ Gender } \\
\hline Male & 89.7 \\
\hline Female & 88.1 \\
\hline \multicolumn{2}{|l|}{ Level of Training } \\
\hline PGY-1 & 85.9 \\
\hline PGY-2 & 88.8 \\
\hline PGY-3 & 88.6 \\
\hline PGY-4+ & 91.7 \\
\hline \multicolumn{2}{|l|}{ Specialty Group } \\
\hline Medical & 89.0 \\
\hline Surgical & 91.6 \\
\hline Hospital-based & 85.4 \\
\hline \multicolumn{2}{|l|}{ CLE Characteristics $^{b}$} \\
\hline \multicolumn{2}{|l|}{ Region $^{c}$} \\
\hline Northeast & 88.9 \\
\hline Midwest & 88.5 \\
\hline South & 86.6 \\
\hline West & 92.0 \\
\hline \multicolumn{2}{|l|}{ Bed Size } \\
\hline$<200$ & 89.5 \\
\hline $200-299$ & 89.1 \\
\hline 300-399 & 86.3 \\
\hline $400-499$ & 87.1 \\
\hline 500 or more & 92.0 \\
\hline \multicolumn{2}{|l|}{ Type of Ownership*** } \\
\hline Nongovernment, not-for-profit & 89.4 \\
\hline Investor-owned, for-profit & 78.6 \\
\hline Government, federal & 89.2 \\
\hline Government, nonfederal & 96.0 \\
\hline
\end{tabular}

DISTRIBUTION ACROSS CLES ${ }^{d}(n=268)$

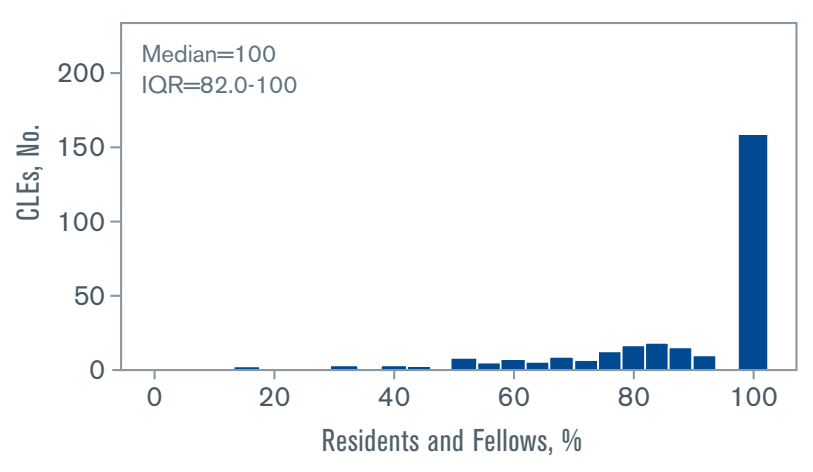

${ }^{a}$ Missing data $(<1 \%)$ have been omitted; percentages based on valid percent.

${ }^{\mathrm{b}}$ Missing data $(<7 \%)$ have been omitted; percentages based on valid percent. Of note, data are missing largely due to clinical sites that do not report data to the American Hospital Association.

${ }^{\mathrm{c}}$ Results from clinical learning environments (CLEs) in Puerto Rico (3\%) omitted to ensure anonymity.

${ }^{\mathrm{d}}$ Distribution includes $95 \%$ or more of the total number of CLEs $(\mathrm{N}=270)$.

* Statistically significant at $P<.05$.

** Statistically significant at $P<.01$.

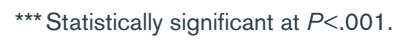

Abbreviation: PGY, postgraduate year. 


\section{B7. Percentage of Residents and Fellows (PGY-2 and Above) Who Reported Knowing the Clinical Site's Priorities in the Area of Quality Improvement}

\section{PERCENT OF TOTAL \\ SURVEYED ${ }^{a}(\mathrm{~N}=1768)$}

\section{3}

\section{PERCENTAGE BY RESIDENT AND \\ FELLOW AND CLE CHARACTERISTICS}

\begin{tabular}{|l|c|}
\hline $\begin{array}{l}\text { Resident and Fellow } \\
\text { Characteristics }^{\mathrm{a}}\end{array}$ & $\begin{array}{c}\text { Residents and Fellows, } \\
\%(\mathrm{n}=1768)\end{array}$ \\
\hline \begin{tabular}{|l|} 
Gender \\
Male
\end{tabular} \\
\hline Female & 84.8 \\
\hline Level of Training & 87.9 \\
\hline PGY-2 & 85.0 \\
\hline PGY-3 & 88.3 \\
\hline PGY-4+ & 84.5 \\
\hline Specialty Group & 86.6 \\
\hline Medical & 86.4 \\
\hline Surgical & 80.8 \\
\hline Hospital-based
\end{tabular}

CLE Characteristics $^{\mathrm{b}}$

\section{Region $^{c}$}

\begin{tabular}{|l|r|}
\hline Northeast & 88.8 \\
\hline Midwest & 83.4 \\
\hline South & 87.3 \\
\hline West & 83.8 \\
\hline
\end{tabular}

\section{Bed Size*}

\begin{tabular}{|l|l|}
\hline$<200$ & 85.3 \\
\hline $200-299$ & 90.6 \\
\hline $300-399$ & 83.1 \\
\hline $400-499$ & 89.2 \\
\hline 500 or more & 84.0 \\
\hline $\begin{array}{l}\text { Type of Ownership*** } \\
\text { Nongovernment, not-for-profit }\end{array}$ \\
\hline Investor-owned, for-profit & 85.9 \\
\hline Government, federal & 85.1 \\
\hline Government, nonfederal & 77.6 \\
\hline
\end{tabular}

\section{DISTRIBUTION ACROSS CLES ${ }^{d}(n=266)$}

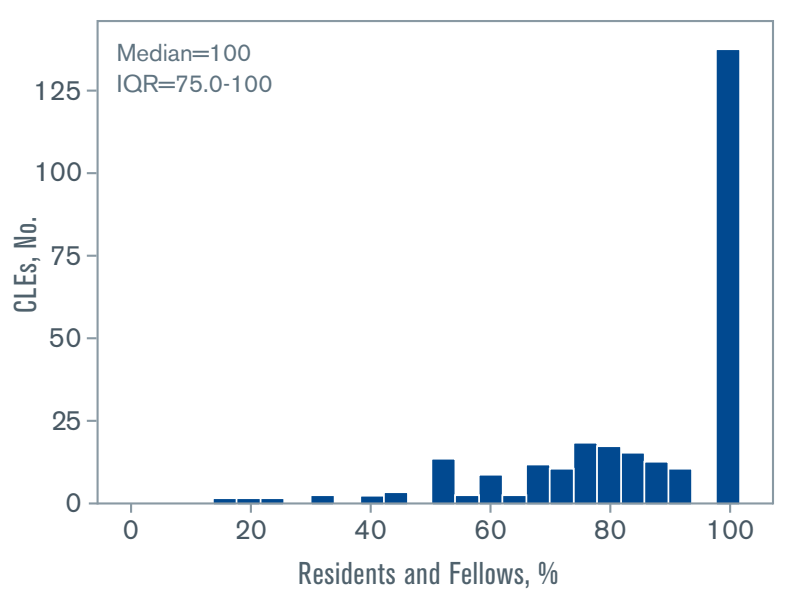

${ }^{a}$ Missing data $(<1 \%)$ have been omitted; percentages based on valid percent.

${ }^{\mathrm{b}}$ Missing data $(<7 \%)$ have been omitted; percentages based on valid percent. Of note, data are missing largely due to clinical sites that do not report data to the American Hospital Association.

${ }^{\circ}$ Results from clinical learning environments (CLEs) in Puerto Rico (3\%) omitted to ensure anonymity.

${ }^{d}$ Distribution includes $95 \%$ or more of the total number of CLEs $(\mathrm{N}=270)$.

* Statistically significant at $P<.05$.

** Statistically significant at $P<.01$.

*** Statistically significant at $P<.001$.

Abbreviation: PGY, postgraduate year. 


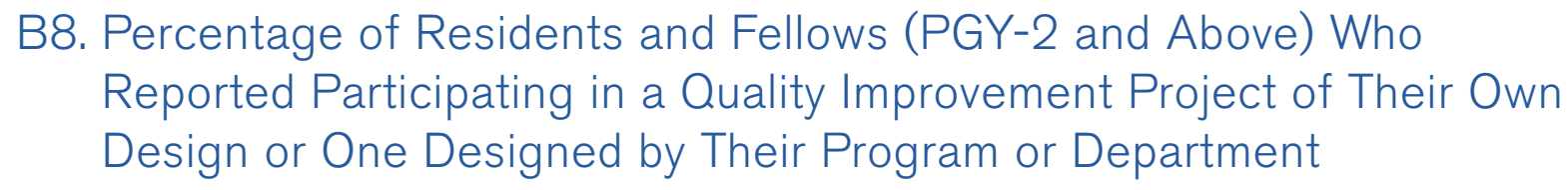

PERCENT OF TOTAL

SURVEYEDa $(N=1730)$

\section{3}

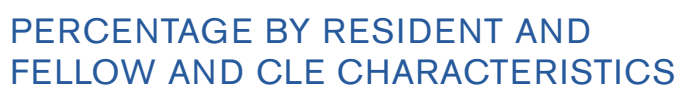

\section{Resident and Fellow}

Characteristics $^{\mathrm{a}}$

Residents and Fellows, $\%(n=1730)$

Gender

Male

Female

80.2

80.4

Level of Training***

PGY-2

PGY-3

PGY-4+

76.1

86.0

76.6

\section{Specialty Group ${ }^{* * *}$}

Medical

81.7

Surgical

66.7

Hospital-based

73.1

\section{CLE Characteristics ${ }^{b}$}

Region $^{\text {c***}}$

\begin{tabular}{l|l} 
Northeast & 82.4 \\
\hline
\end{tabular}

Midwest

78.0

South

West

80.5

Bed Size**

$<200$

85.3

200-299

75.0

300-399

78.8

400-499

82.1

500 or more

76.5

\section{Type of Ownership}

Nongovernment, not-for-profit

79.2

Investor-owned, for-profit

82.7

Government, federal

78.6

Government, nonfederal
82.8
DISTRIBUTION ACROSS CLEs ${ }^{d}(n=263)$

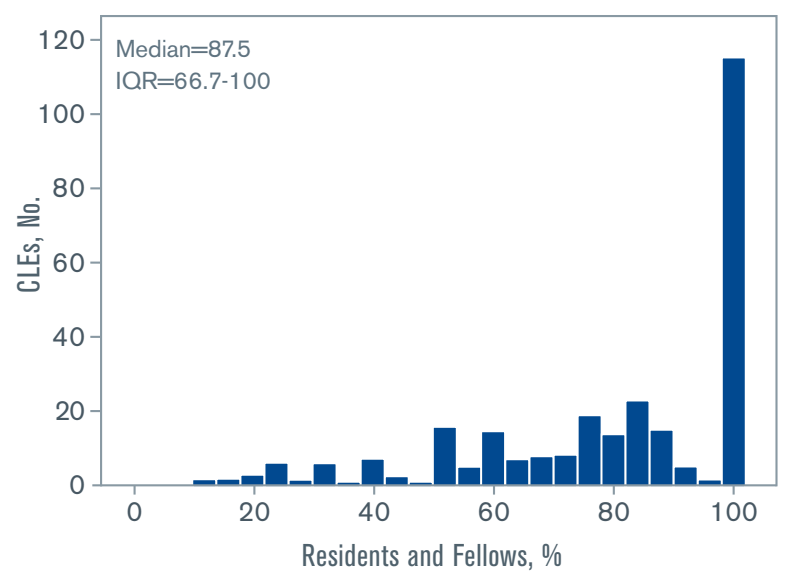

${ }^{a}$ Missing data $(<3 \%)$ have been omitted; percentages based on valid percent.

${ }^{\mathrm{b}}$ Missing data $(<9 \%)$ have been omitted; percentages based on valid percent. Of note, data are missing largely due to clinical sites that do not report data to the American Hospital Association.

${ }^{\mathrm{c}}$ Results from clinical learning environments (CLEs) in Puerto Rico (3\%) omitted to ensure anonymity.

${ }^{d}$ Distribution includes $95 \%$ or more of the total number of CLEs $(\mathrm{N}=270)$.

* Statistically significant at $P<.05$.

** Statistically significant at $P<.01$.

*** Statistically significant at $P<.001$.

Abbreviation: PGY, postgraduate year. 
B9. Percentage of Residents and Fellows (PGY-2 and Above) Who Reported Participating in a Quality Improvement Project Linked to 1 or More of the Clinical Site's Quality Improvement Goals

PERCENT OF TOTAL

SURVEYED ${ }^{a}(\mathrm{~N}=1367)$

\section{7}

\section{PERCENTAGE BY RESIDENT AND \\ FELLOW AND CLE CHARACTERISTICS}

\begin{tabular}{|l|c|}
\hline $\begin{array}{l}\text { Resident and Fellow } \\
\text { Characteristics }^{\mathrm{a}}\end{array}$ & $\begin{array}{c}\text { Residents and Fellows, } \\
\%(\mathrm{n}=1367)\end{array}$ \\
\hline \begin{tabular}{|l|} 
Gender \\
Male
\end{tabular} \\
\hline Female & 59.9 \\
\hline Level of Training & 61.6 \\
\hline PGY-2 & 60.8 \\
\hline PGY-3 & 62.5 \\
\hline PGY-4+ & 52.7 \\
\hline Specialty Group* & \\
\hline Medical & 61.1 \\
\hline Surgical & 66.7 \\
\hline Hospital-based & 44.6 \\
\hline
\end{tabular}

\section{CLE Characteristics ${ }^{b}$}

\section{Region $^{\text {**** }}$}

\begin{tabular}{|l|l|}
\hline Northeast & 69.1 \\
\hline Midwest & 53.2 \\
\hline South & 61.5 \\
\hline West & 50.0 \\
\hline
\end{tabular}

\begin{tabular}{|l|l|}
\hline \begin{tabular}{|l|} 
Bed Size \\
\hline$<200$
\end{tabular} & 65.6 \\
\hline $200-299$ & 56.3 \\
\hline $300-399$ & 58.5 \\
\hline $400-499$ & 56.1 \\
\hline 500 or more & 58.3 \\
\hline Type of Ownership*** \\
\hline Nongovernment, not-for-profit \\
\hline Investor-owned, for-profit & 58.2 \\
\hline Government, federal & 58.7 \\
\hline Government, nonfederal & 54.1 \\
\hline
\end{tabular}

DISTRIBUTION ACROSS CLES ${ }^{d}(n=263)$

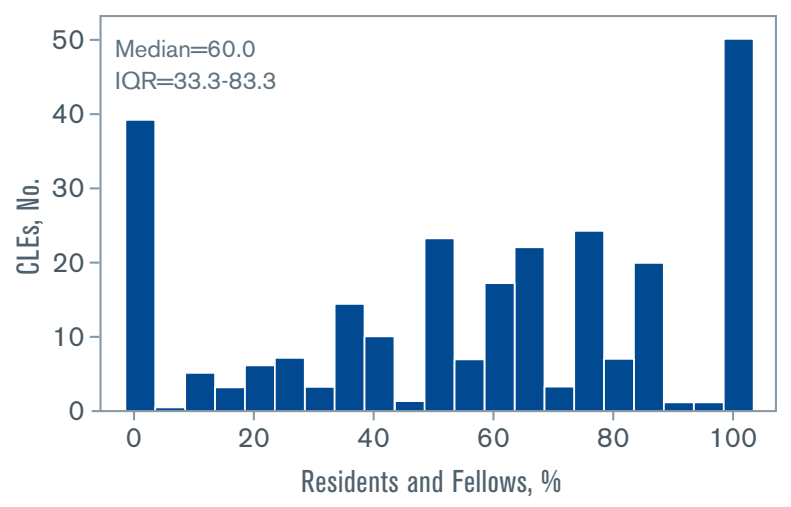

${ }^{a}$ Missing data $(<2 \%)$ have been omitted; percentages based on valid percent.

${ }^{\mathrm{b}}$ Missing data $(<8 \%)$ have been omitted; percentages based on valid percent. Of note, data are missing largely due to clinical sites that do not report data to the American Hospital Association.

${ }^{\circ}$ Results from clinical learning environments (CLEs) in Puerto Rico (3\%) omitted to ensure anonymity.

${ }^{\mathrm{d}}$ Distribution includes $95 \%$ or more of the total number of CLEs $(\mathrm{N}=270)$.

* Statistically significant at $P<.05$.

** Statistically significant at $P<.01$.

*** Statistically significant at $P<.001$.

Abbreviation: PGY, postgraduate year. 
B10. Percentage of Residents and Fellows (PGY-2 and Above) Who Reported Being Engaged in Interprofessional Quality Improvement Teams (eg, Nurses, Administrators, Pharmacists, etc) While Participating in a Quality Improvement Project Directly Linked to 1 or More of the Clinical Site's Quality Improvement Goals

PERCENT OF TOTAL

SURVEYED ${ }^{\mathrm{a}}(\mathrm{N}=818)$

\section{PERCENTAGE BY RESIDENT AND \\ FELLOW AND CLE CHARACTERISTICS}

\begin{tabular}{|c|c|}
\hline $\begin{array}{l}\text { Resident and Fellow } \\
\text { Characteristics }^{\text {a }}\end{array}$ & $\begin{array}{l}\text { Residents and Fellows, } \\
\qquad \%(n=818)\end{array}$ \\
\hline \multicolumn{2}{|l|}{ Gender } \\
\hline Male & 82.7 \\
\hline Female & 84.1 \\
\hline \multicolumn{2}{|l|}{ Level of Training } \\
\hline PGY-2 & 82.4 \\
\hline PGY-3 & 84.7 \\
\hline PGY-4+ & 81.6 \\
\hline \multicolumn{2}{|l|}{ Specialty Group } \\
\hline Medical & 83.5 \\
\hline Surgical & 85.7 \\
\hline Hospital-based & 76.0 \\
\hline \multicolumn{2}{|l|}{ CLE Characteristics $^{b}$} \\
\hline \multicolumn{2}{|l|}{ Region $^{c}$} \\
\hline Northeast & 82.9 \\
\hline Midwest & 87.8 \\
\hline South & 81.3 \\
\hline West & 80.0 \\
\hline \multicolumn{2}{|l|}{ Bed Size } \\
\hline$<200$ & 81.8 \\
\hline $200-299$ & 83.9 \\
\hline $300-399$ & 83.9 \\
\hline $400-499$ & 86.0 \\
\hline 500 or more & 81.6 \\
\hline \multicolumn{2}{|l|}{ Type of Ownership* } \\
\hline Nongovernment, not-for-profit & 84.7 \\
\hline Investor-owned, for-profit & 85.7 \\
\hline Government, federal & 69.2 \\
\hline Government, nonfederal & 78.9 \\
\hline
\end{tabular}

DISTRIBUTION ACROSS CLES ${ }^{d}(n=221)$

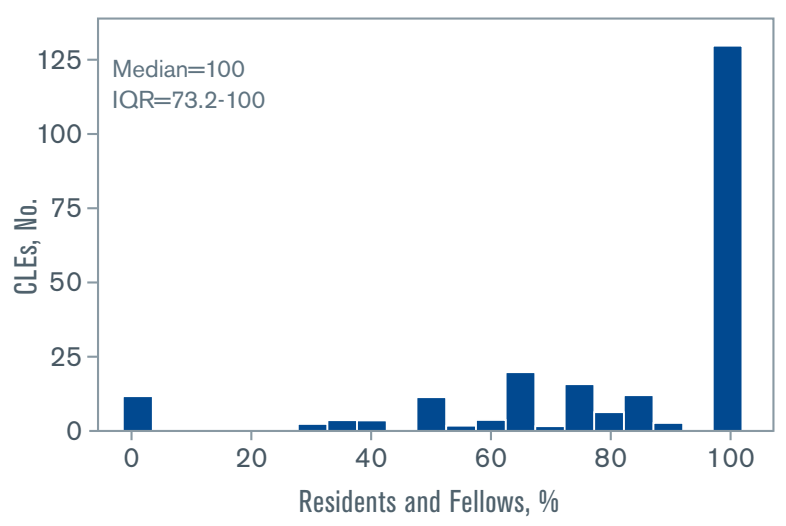

${ }^{\text {a }}$ Missing data $(<2 \%)$ have been omitted; percentages based on valid percent.

${ }^{\mathrm{b}}$ Missing data $(<10 \%)$ have been omitted; percentages based on valid percent. Of note, data are missing largely due to clinical sites that do not report data to the American Hospital Association.

${ }^{\mathrm{c}}$ Results from clinical learning environments (CLEs) in Puerto Rico (3\%) omitted to ensure anonymity.

${ }^{\mathrm{d}}$ Distribution includes $95 \%$ or more of the total number of CLEs $(\mathrm{N}=270)$.

* Statistically significant at $P<.05$.

** Statistically significant at $P<.01$.

*** Statistically significant at $P<.001$.

Abbreviation: PGY, postgraduate year. 


\section{B11. Percentage of Residents and Fellows Who Reported Receiving Aggregated or Benchmarked Quality Performance Data About the Care of Their Own Patients}

PERCENT OF TOTAL

SURVEYEDa $(\mathrm{N}=1801)$

\section{2}

\section{PERCENTAGE BY RESIDENT AND FELLOW AND CLE CHARACTERISTICS}

\begin{tabular}{|c|c|}
\hline $\begin{array}{l}\text { Resident and Fellow } \\
\text { Characteristics }^{\text {a }}\end{array}$ & $\begin{array}{l}\text { Residents and Fellows, } \\
\qquad \%(n=1801)\end{array}$ \\
\hline \multicolumn{2}{|l|}{ Gender } \\
\hline Male & 52.3 \\
\hline Female & 54.2 \\
\hline \multicolumn{2}{|l|}{ Level of Training*** } \\
\hline PGY-1 & 28.1 \\
\hline PGY-2 & 55.5 \\
\hline PGY-3 & 56.5 \\
\hline PGY-4+ & 40.0 \\
\hline \multicolumn{2}{|l|}{ Specialty Group*** } \\
\hline Medical & 55.6 \\
\hline Surgical & 39.1 \\
\hline Hospital-based & 36.6 \\
\hline \multicolumn{2}{|l|}{ CLE Characteristics $^{\mathrm{b}}$} \\
\hline \multicolumn{2}{|l|}{ Regionc } \\
\hline Northeast & 47.0 \\
\hline Midwest & 51.9 \\
\hline South & 52.7 \\
\hline West & 56.3 \\
\hline \multicolumn{2}{|l|}{ Bed Size } \\
\hline$<200$ & 53.4 \\
\hline $200-299$ & 48.3 \\
\hline 300-399 & 52.1 \\
\hline $400-499$ & 49.6 \\
\hline 500 or more & 53.8 \\
\hline \multicolumn{2}{|l|}{ Type of Ownership ${ }^{* * *}$} \\
\hline Nongovernment, not-for-profit & 53.0 \\
\hline Investor-owned, for-profit & 32.6 \\
\hline Government, federal & 61.8 \\
\hline Government, nonfederal & 59.1 \\
\hline
\end{tabular}

DISTRIBUTION ACROSS CLES ${ }^{d}(n=265)$

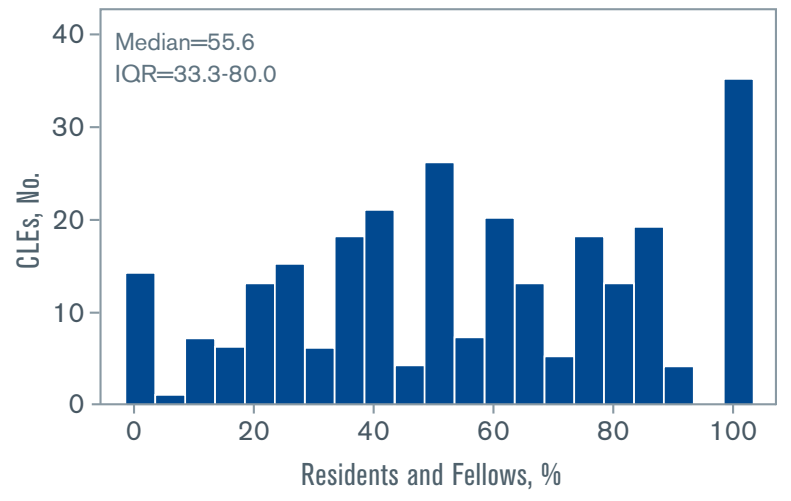

a Missing data $(<3 \%)$ have been omitted; percentages based on valid percent.

${ }^{b}$ Missing data (<8\%) have been omitted; percentages based on valid percent. Of note, data are missing largely due to clinical sites that do not report data to the American Hospital Association.

${ }^{\mathrm{c}}$ Results from clinical learning environments (CLEs) in Puerto Rico (3\%) omitted to ensure anonymity.

${ }^{d}$ Distribution includes $95 \%$ or more of the total number of CLEs $(\mathrm{N}=270)$.

* Statistically significant at $P<.05$.

** Statistically significant at $P<.01$.

*** Statistically significant at $P<.001$.

Abbreviation: PGY, postgraduate year. 


\section{B12. Percentage of Residents and Fellows Who Reported Knowing the Clinical Site's Priorities With Regard to Addressing Health Care Disparities}

PERCENT OF TOTAL

SURVEYED ${ }^{a}(\mathrm{~N}=1839)$

\section{7}

\section{PERCENTAGE BY RESIDENT AND \\ FELLOW AND CLE CHARACTERISTICS}

Resident and Fellow

Characteristics $^{\mathrm{a}}$

Residents and Fellows, $\%(n=1839)$

\section{Gender*}

Male

Female

63.2

68.8

\section{Level of Training}

PGY-1

PGY-2

PGY-3

PGY-4+

Specialty Group*

Medical

Surgical

Hospital-based

CLE Characteristics $^{\mathrm{b}}$

\section{Region $^{\text {c***}}$}

\begin{tabular}{|l|l|}
\hline Northeast & 72.0 \\
\hline Midwest & 60.2 \\
\hline South & 64.0 \\
\hline West & 63.0 \\
\hline
\end{tabular}

\section{Bed Size **}

$$
<200
$$

200-299

300-399

400-499

500 or more

73.0

63.2

62.6

61.3

63.1

\section{Type of Ownership ${ }^{* * *}$}

Nongovernment, not-for-profit

65.2

Investor-owned, for-profit

56.3

Government, federal

52.9

Government, nonfederal
56.3

64.2

69.9

66.2

71.4

56.1 


\section{B13. Percentage of Residents And Fellows Who Reported Receiving Cultural Competency Training Specific to Populations at Risk for Health Care Disparities at Their Clinical Site}

PERCENT OF TOTAL

SURVEYED ${ }^{a}(\mathrm{~N}=1833)$

\section{6}

\section{PERCENTAGE BY RESIDENT AND \\ FELLOW AND CLE CHARACTERISTICS}

\begin{tabular}{|c|c|}
\hline $\begin{array}{l}\text { Resident and Fellow } \\
\text { Characteristics }^{\mathrm{a}}\end{array}$ & $\begin{array}{c}\text { Residents and Fellows, } \\
\qquad \%(n=1833)\end{array}$ \\
\hline \multicolumn{2}{|l|}{ Gender } \\
\hline Male & 45.3 \\
\hline Female & 48.0 \\
\hline \multicolumn{2}{|l|}{ Level of Training } \\
\hline PGY-1 & 42.2 \\
\hline PGY-2 & 46.8 \\
\hline PGY-3 & 47.2 \\
\hline PGY-4+ & 44.7 \\
\hline \multicolumn{2}{|l|}{ Specialty Group } \\
\hline Medical & 47.2 \\
\hline Surgical & 42.0 \\
\hline Hospital-based & 43.1 \\
\hline \multicolumn{2}{|l|}{ CLE Characteristics $^{\text {b }}$} \\
\hline \multicolumn{2}{|l|}{ Region $^{c * *}$} \\
\hline Northeast & 47.4 \\
\hline Midwest & 42.5 \\
\hline South & 42.6 \\
\hline West & 51.5 \\
\hline \multicolumn{2}{|l|}{ Bed Size } \\
\hline$<200$ & 50.1 \\
\hline $200-299$ & 44.7 \\
\hline $300-399$ & 47.0 \\
\hline $400-499$ & 37.9 \\
\hline 500 or more & 46.4 \\
\hline \multicolumn{2}{|l|}{ Type of Ownership ${ }^{* * *}$} \\
\hline Nongovernment, not-for-profit & 44.6 \\
\hline Investor-owned, for-profit & 34.8 \\
\hline Government, federal & 47.1 \\
\hline Government, nonfederal & 66.2 \\
\hline
\end{tabular}

DISTRIBUTION ACROSS CLEs ${ }^{d}(n=268)$

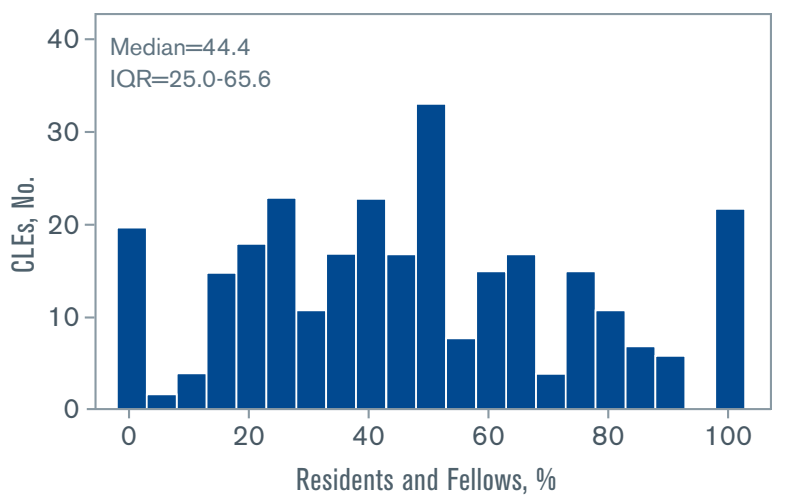

aissing data $(<1 \%)$ have been omitted; percentages based on valid percent.

${ }^{\mathrm{b}}$ Missing data $(<7 \%)$ have been omitted; percentages based on valid percent. Of note, data are missing largely due to clinical sites that do not report data to the American Hospital Association.

${ }^{\circ}$ Results from clinical learning environments (CLEs) in Puerto Rico (3\%) omitted to ensure anonymity.

${ }^{d}$ Distribution includes $95 \%$ or more of the total number of CLEs $(\mathrm{N}=270)$.

* Statistically significant at $P<.05$.

** Statistically significant at $P<.01$.

${ }^{* * *}$ Statistically significant at $P<.001$.

Abbreviation: PGY, postgraduate year. 


\section{B14. Percentage of Residents and Fellows Who Reported Participating In a Quality Improvement Project Focused on Reducing Health Care Disparities}

PERCENT OF TOTAL

SURVEYED ${ }^{a}(\mathrm{~N}=1838)$

\section{4}

\section{PERCENTAGE BY RESIDENT AND \\ FELLOW AND CLE CHARACTERISTICS}

\begin{tabular}{|c|c|}
\hline $\begin{array}{l}\text { Resident and Fellow } \\
\text { Characteristics }^{\mathrm{a}}\end{array}$ & $\begin{array}{l}\text { Residents and Fellows, } \\
\qquad \%(n=1838)\end{array}$ \\
\hline \multicolumn{2}{|l|}{ Gender** } \\
\hline Male & 14.8 \\
\hline Female & 20.2 \\
\hline \multicolumn{2}{|l|}{ Level of Training ${ }^{* \star *}$} \\
\hline PGY-1 & 3.1 \\
\hline PGY-2 & 17.0 \\
\hline PGY-3 & 20.4 \\
\hline PGY-4+ & 12.6 \\
\hline \multicolumn{2}{|l|}{ Specialty Group ${ }^{* * *}$} \\
\hline Medical & 18.9 \\
\hline Surgical & 9.2 \\
\hline Hospital-based & 5.7 \\
\hline \multicolumn{2}{|l|}{ CLE Characteristics $^{b}$} \\
\hline \multicolumn{2}{|l|}{ Region $^{c * *}$} \\
\hline Northeast & 19.0 \\
\hline Midwest & 10.8 \\
\hline South & 17.3 \\
\hline West & 22.7 \\
\hline \multicolumn{2}{|l|}{ Bed Size } \\
\hline$<200$ & 17.7 \\
\hline $200-299$ & 17.7 \\
\hline 300-399 & 17.3 \\
\hline $400-499$ & 18.4 \\
\hline 500 or more & 14.8 \\
\hline \multicolumn{2}{|l|}{ Type of Ownership*** } \\
\hline Nongovernment, not-for-profit & 16.7 \\
\hline Investor-owned, for-profit & 18.8 \\
\hline Government, federal & 3.9 \\
\hline Government, nonfederal & 25.8 \\
\hline
\end{tabular}

DISTRIBUTION ACROSS CLES ${ }^{d}(n=268)$

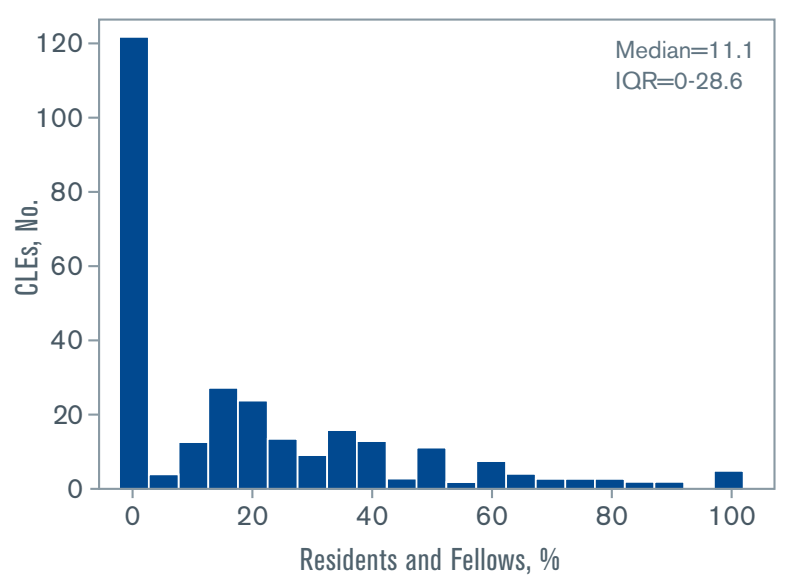

${ }^{a}$ Missing data $(<1 \%)$ have been omitted; percentages based on valid percent.

${ }^{\mathrm{b}}$ Missing data $(<7 \%)$ have been omitted; percentages based on valid percent. Of note, data are missing largely due to clinical sites that do not report data to the American Hospital Association.

${ }^{\mathrm{c}}$ Results from clinical learning environments (CLEs) in Puerto Rico (3\%) omitted to ensure anonymity.

${ }^{\mathrm{d}}$ Distribution includes $95 \%$ or more of the total number of CLEs $(\mathrm{N}=270)$.

* Statistically significant at $P<.05$.

** Statistically significant at $P<.01$.

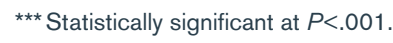

Abbreviation: PGY, postgraduate year. 


\section{B15. Percentage of Residents and Fellows Who Reported Participating in Training With Nurses and Other Health Care Professionals in How to Transition Patients' Care}

PERCENT OF TOTAL

SURVEYED ${ }^{a}(\mathrm{~N}=1833)$

\section{8}

\section{PERCENTAGE BY RESIDENT AND \\ FELLOW AND CLE CHARACTERISTICS}

\begin{tabular}{|c|c|}
\hline $\begin{array}{l}\text { Resident and Fellow } \\
\text { Characteristics }^{\mathrm{a}}\end{array}$ & $\begin{array}{c}\text { Residents and Fellows, } \\
\%(n=1833)\end{array}$ \\
\hline \multicolumn{2}{|l|}{ Gender } \\
\hline Male & 75.1 \\
\hline Female & 76.6 \\
\hline \multicolumn{2}{|l|}{ Level of Training } \\
\hline PGY-1 & 62.5 \\
\hline PGY-2 & 75.4 \\
\hline PGY-3 & 76.9 \\
\hline PGY-4+ & 77.5 \\
\hline \multicolumn{2}{|l|}{ Specialty Group** } \\
\hline Medical & 77.0 \\
\hline Surgical & 71.4 \\
\hline Hospital-based & 64.8 \\
\hline \multicolumn{2}{|l|}{ CLE Characteristics $^{b}$} \\
\hline \multicolumn{2}{|l|}{ Region ${ }^{*}$} \\
\hline Northeast & 78.9 \\
\hline Midwest & 71.1 \\
\hline South & 76.4 \\
\hline West & 75.2 \\
\hline \multicolumn{2}{|l|}{ Bed Size $^{*}$} \\
\hline$<200$ & 80.7 \\
\hline $200-299$ & 75.7 \\
\hline $300-399$ & 75.4 \\
\hline $400-499$ & 71.9 \\
\hline 500 or more & 71.9 \\
\hline \multicolumn{2}{|l|}{ Type of Ownership*** } \\
\hline Nongovernment, not-for-profit & 74.7 \\
\hline Investor-owned, for-profit & 69.5 \\
\hline Government, federal & 84.3 \\
\hline Government, nonfederal & 85.9 \\
\hline
\end{tabular}

DISTRIBUTION ACROSS CLEs ${ }^{d}(n=268)$

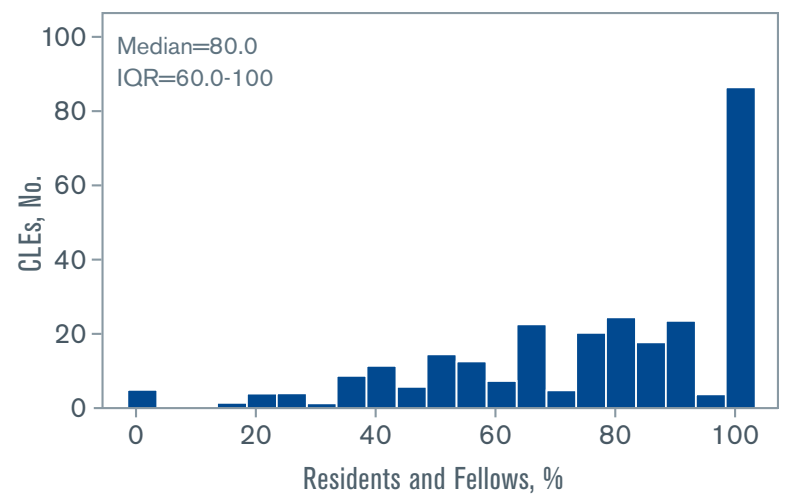

${ }^{a}$ Missing data $(<1 \%)$ have been omitted; percentages based on valid percent.

${ }^{\mathrm{b}}$ Missing data $(<7 \%)$ have been omitted; percentages based on valid percent. Of note, data are missing largely due to clinical sites that do not report data to the American Hospital Association.

${ }^{\circ}$ Results from clinical learning environments (CLEs) in Puerto Rico (3\%) omitted to ensure anonymity.

${ }^{d}$ Distribution includes $95 \%$ or more of the total number of CLEs $(\mathrm{N}=270)$.

* Statistically significant at $P<.05$.

** Statistically significant at $P<.01$.

*** Statistically significant at $P<.001$.

Abbreviation: PGY, postgraduate year. 


\section{B16. Percentage of Residents and Fellows Who Reported Following Standardized Processes for Handling Transitions of Care From Inpatient to Outpatient}

PERCENT OF TOTAL

SURVEYED ${ }^{a}(\mathrm{~N}=1648)$

\section{6}

\section{PERCENTAGE BY RESIDENT AND \\ FELLOW AND CLE CHARACTERISTICS}

\begin{tabular}{|l|c|}
\hline $\begin{array}{l}\text { Resident and Fellow } \\
\text { Characteristics }\end{array}$ & $\begin{array}{c}\text { Residents and Fellows, } \\
\%(n=1648)\end{array}$ \\
\hline $\begin{array}{l}\text { Gender } \\
\text { Male }\end{array}$ \\
\hline \begin{tabular}{l} 
Female \\
Level of Training* \\
\hline PGY-1
\end{tabular} \\
\hline PGY-2 & 80.3 \\
\hline PGY-3 & 82.9 \\
\hline PGY-4+ & 76.2 \\
\hline Specialty Group & \\
\hline Medical & 83.5 \\
\hline Surgical & 81.8 \\
\hline Hospital-based & 74.4 \\
\hline CLE Characteristics & \\
\hline Region & \\
\hline Northeast & 83.3 \\
\hline Midwest & 77.0 \\
\hline South & 64.0 \\
\hline West & \\
\hline Bed Size*** & \\
\hline$<200$ & 82.3 \\
\hline $200-299$ & 81.7 \\
\hline $300-399$ & 80.8 \\
\hline $400-499$ & 80.8 \\
\hline 500 or more $^{*}$ & 83.9 \\
\hline Type of Ownership*** & 79.9 \\
\hline Nongovernment, not-for-profit & 86.8 \\
\hline Investor-owned, for-profit & 70.7 \\
\hline Government, federal & 82.1 \\
\hline Government, nonfederal & 82.3 \\
\hline & 80.2 \\
\hline
\end{tabular}

DISTRIBUTION ACROSS CLEs ${ }^{d}(n=240)$

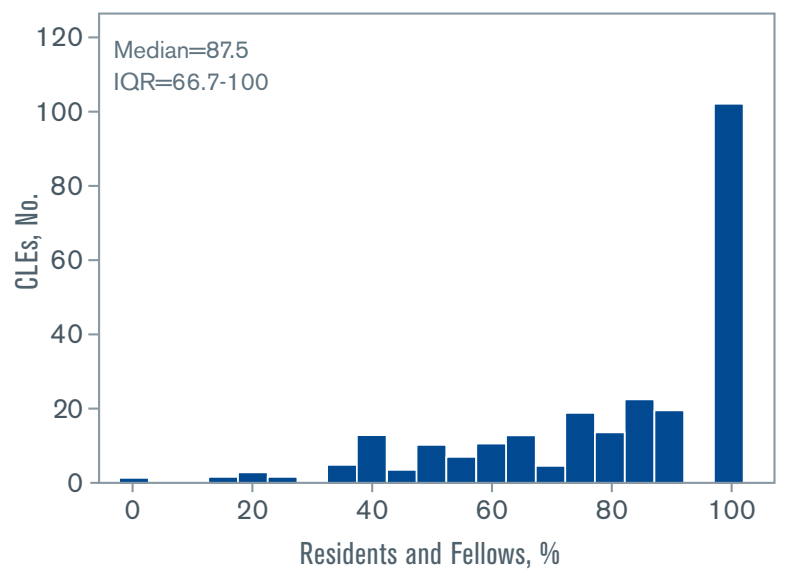

${ }^{a}$ Missing data $(<4 \%)$ have been omitted; percentages based on valid percent.

${ }^{\mathrm{b}}$ Missing data $(<4 \%)$ have been omitted; percentages based on valid percent. Of note, data are missing largely due to clinical sites that do not report data to the American Hospital Association.

${ }^{\mathrm{c}}$ Results from clinical learning environments (CLEs) in Puerto Rico (3\%) omitted to ensure anonymity.

${ }^{\mathrm{d}}$ Distribution includes $95 \%$ or more of the total number of CLEs $(\mathrm{N}=246)$. Of note, results from visits that were held exclusively in the ambulatory care setting ( $9 \%$ ) have been omitted due to modifications to the question and/or scenario to better fit the setting; these modifications limited comparability across inpatient and ambulatory care settings.

* Statistically significant at $P<.05$.

** Statistically significant at $P<.01$.

*** Statistically significant at $P<.001$.

Abbreviation: PGY, postgraduate year. 


\section{B17. Percentage of Residents and Fellows Who Reported Following a Standardized Process for Handling Transitions of Care During Handoffs Between Shifts}

PERCENT OF TOTAL

SURVEYED ${ }^{a}(\mathrm{~N}=1658)$

\section{9}

\section{PERCENTAGE BY RESIDENT AND \\ FELLOW AND CLE CHARACTERISTICS}

\begin{tabular}{|c|c|}
\hline $\begin{array}{l}\text { Resident and Fellow } \\
\text { Characteristics }^{\mathrm{a}}\end{array}$ & $\begin{array}{c}\text { Residents and Fellows, } \\
\qquad \%(n=1658)\end{array}$ \\
\hline \multicolumn{2}{|l|}{ Gender } \\
\hline Male & 95.0 \\
\hline Female & 94.8 \\
\hline \multicolumn{2}{|l|}{ Level of Training*** } \\
\hline PGY-1 & 93.8 \\
\hline PGY-2 & 95.7 \\
\hline PGY-3 & 96.2 \\
\hline PGY-4+ & 87.2 \\
\hline \multicolumn{2}{|l|}{ Specialty Group*** } \\
\hline Medical & 96.4 \\
\hline Surgical & 86.7 \\
\hline Hospital-based & 85.2 \\
\hline \multicolumn{2}{|l|}{ CLE Characteristics $^{\mathrm{b}}$} \\
\hline \multicolumn{2}{|l|}{ Regionc } \\
\hline Northeast & 94.7 \\
\hline Midwest & 94.3 \\
\hline South & 94.7 \\
\hline West & 95.5 \\
\hline \multicolumn{2}{|l|}{ Bed Size } \\
\hline$<200$ & 96.4 \\
\hline $200-299$ & 94.0 \\
\hline 300-399 & 95.8 \\
\hline $400-499$ & 93.5 \\
\hline 500 or more & 93.3 \\
\hline \multicolumn{2}{|l|}{ Type of Ownership* } \\
\hline Nongovernment, not-for-profit & 94.2 \\
\hline Investor-owned, for-profit & 93.5 \\
\hline Government, federal & 98.0 \\
\hline Government, nonfederal & 99.0 \\
\hline
\end{tabular}

DISTRIBUTION ACROSS CLEs ${ }^{d}(n=241)$

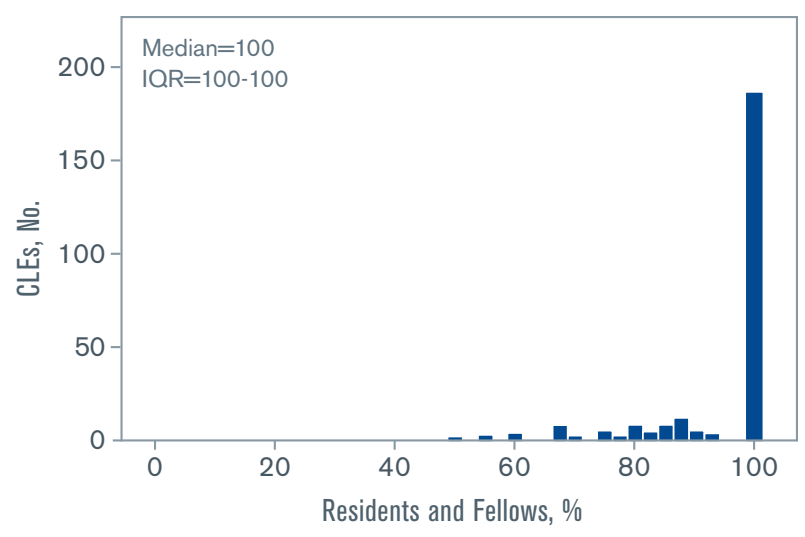

${ }^{\text {a }}$ Missing data $(<3 \%)$ have been omitted; percentages based on valid percent.

${ }^{\mathrm{b}}$ Missing data $(<3 \%)$ have been omitted; percentages based on valid percent. Of note, data are missing largely due to clinical sites that do not report data to the American Hospital Association.

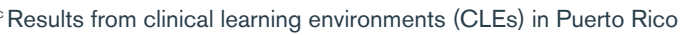
(3\%) omitted to ensure anonymity.

${ }^{d}$ Distribution includes $95 \%$ or more of the total number of CLEs $(\mathrm{N}=246)$. Of note, results from visits that were held exclusively in the ambulatory care setting (9\%) have been omitted due to modifications to the question and/or scenario to better fit the setting; these modifications limited comparability across inpatient and ambulatory care settings.

* Statistically significant at $P<.05$.

** Statistically significant at $P<.01$.

*** Statistically significant at $P<.001$.

Abbreviation: PGY, postgraduate year. 


\section{B18. Percentage of Residents and Fellows Who Reported Following a Standardized Process for Handoffs Between Shifts That Included a Standardized Written Template for Communication}

PERCENT OF TOTAL

SURVEYED ${ }^{a}(\mathrm{~N}=1568)$

\section{1}

\section{PERCENTAGE BY RESIDENT AND \\ FELLOW AND CLE CHARACTERISTICS}

\begin{tabular}{|c|c|}
\hline $\begin{array}{l}\text { Resident and Fellow } \\
\text { Characteristics }^{\mathrm{a}}\end{array}$ & $\begin{array}{l}\text { Residents and Fellows } \\
\%(\mathrm{n}=1568)\end{array}$ \\
\hline \multicolumn{2}{|l|}{ Gender } \\
\hline Male & 86.9 \\
\hline Female & 89.8 \\
\hline \multicolumn{2}{|l|}{ Level of Training ${ }^{* *}$} \\
\hline PGY-1 & 83.1 \\
\hline PGY-2 & 88.3 \\
\hline PGY-3 & 90.5 \\
\hline PGY-4+ & 81.2 \\
\hline \multicolumn{2}{|l|}{ Specialty Group* } \\
\hline Medical & 89.1 \\
\hline Surgical & 84.5 \\
\hline Hospital-based & 81.6 \\
\hline \multicolumn{2}{|l|}{ CLE Characteristics $^{\mathrm{b}}$} \\
\hline \multicolumn{2}{|l|}{ Region $^{c *}$} \\
\hline Northeast & 90.4 \\
\hline Midwest & 85.6 \\
\hline South & 87.5 \\
\hline West & 87.0 \\
\hline \multicolumn{2}{|l|}{ Bed Size ${ }^{\star \star *}$} \\
\hline$<200$ & 92.4 \\
\hline $200-299$ & 87.5 \\
\hline 300-399 & 89.5 \\
\hline $400-499$ & 80.6 \\
\hline 500 or more & 86.1 \\
\hline \multicolumn{2}{|l|}{ Type of Ownership } \\
\hline Nongovernment, not-for-profit & 87.2 \\
\hline Investor-owned, for-profit & 87.6 \\
\hline Government, federal & 93.0 \\
\hline Government, nonfederal & 91.7 \\
\hline
\end{tabular}

DISTRIBUTION ACROSS CLEs ${ }^{d}(n=241)$

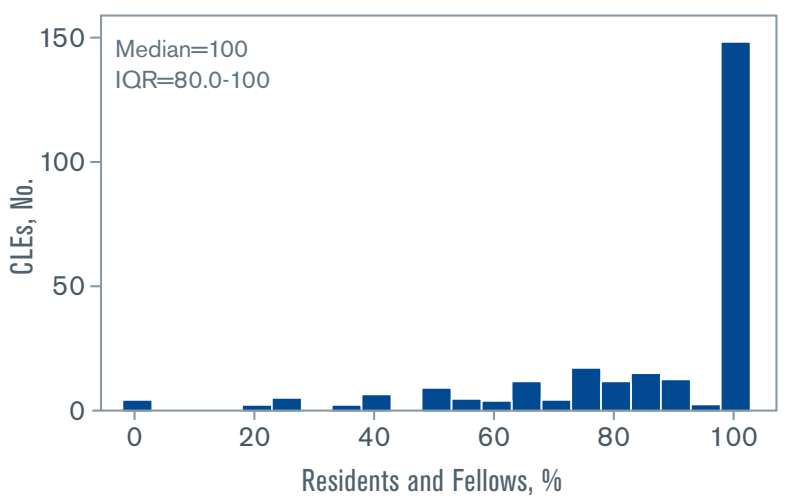

${ }^{a}$ Missing data $(<1 \%)$ have been omitted; percentages based on valid percent.

${ }^{\mathrm{b}}$ Missing data $(<1 \%)$ have been omitted; percentages based on valid percent. Of note, data are missing largely due to clinical sites that do not report data to the American Hospital Association.

${ }^{\mathrm{c}}$ Results from clinical learning environments (CLEs) in Puerto Rico (3\%) omitted to ensure anonymity.

${ }^{\mathrm{d}}$ Distribution includes $95 \%$ or more of the total number of CLEs $(\mathrm{N}=246)$. Of note, results from visits that were held exclusively in the ambulatory care setting ( $9 \%$ ) have been omitted due to modifications to the question and/or scenario to better fit the setting; these modifications limited comparability across inpatient and ambulatory care settings.

* Statistically significant at $P<.05$.

** Statistically significant at $P<.01$.

*** Statistically significant at $P<.001$.

Abbreviation: PGY, postgraduate year. 
B19. Percentage of Residents and Fellows Who Reported Having Been Placed, or Witnessing One of Their Peers Placed, in a Situation Where They Believed There Was Inadequate Supervision at the Clinical Site (eg, the Attending Was Not Available)

PERCENT OF TOTAL

SURVEYED ${ }^{a}(\mathrm{~N}=1837)$

\section{6}

\section{PERCENTAGE BY RESIDENT AND \\ FELLOW AND CLE CHARACTERISTICS}

\begin{tabular}{|l|l|}
\hline $\begin{array}{l}\text { Resident and Fellow } \\
\text { Characteristics }\end{array}$ & $\begin{array}{c}\text { Residents and Fellows, } \\
\text { \% }\end{array}$ \\
\hline Gender $^{* * *}$ & \\
\hline Male & 24.4 \\
\hline Female
\end{tabular}

DISTRIBUTION ACROSS CLES ${ }^{d}(n=268)$

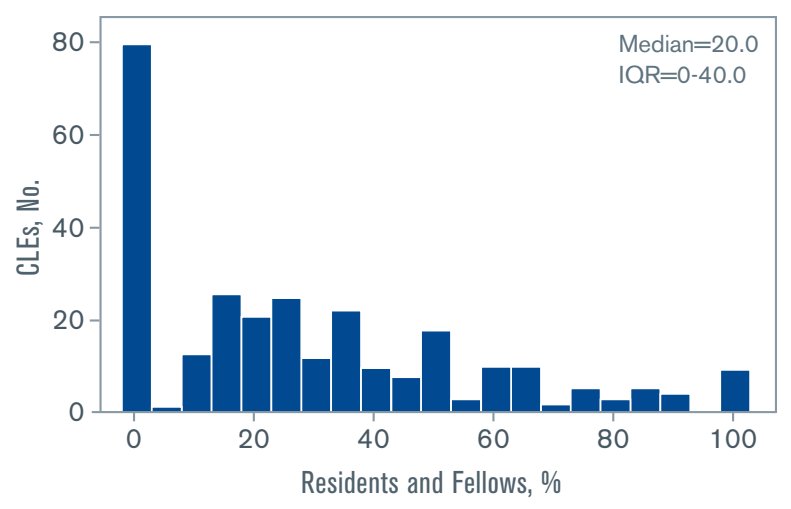

${ }^{a}$ Missing data $(<1 \%)$ have been omitted; percentages based on valid percent.

${ }^{\mathrm{b}}$ Missing data $(<7 \%)$ have been omitted; percentages based on valid percent. Of note, data are missing largely due to clinical sites that do not report data to the American Hospital Association.

${ }^{\mathrm{C}}$ Results from clinical learning environments (CLEs) in Puerto Rico (3\%) omitted to ensure anonymity.

${ }^{d}$ Distribution includes $95 \%$ or more of the total number of CLEs $(\mathrm{N}=270)$.

* Statistically significant at $P<.05$.

** Statistically significant at $P<.01$.

*** Statistically significant at $P<.001$.

Abbreviation: PGY, postgraduate year. 


\section{B20. Percentage of Residents and Fellows Who Reported Encountering a Physician (Attending Physicians or Consultants) Who Made Them Feel Uncomfortable When Requesting Assistance}

PERCENT OF TOTAL

SURVEYED ${ }^{a}(\mathrm{~N}=1836)$

\section{1}

\section{PERCENTAGE BY RESIDENT AND \\ FELLOW AND CLE CHARACTERISTICS}

\begin{tabular}{|c|c|}
\hline $\begin{array}{l}\text { Resident and Fellow } \\
\text { Characteristics }\end{array}$ & $\begin{array}{l}\text { Residents and Fellows, } \\
\%(n=1836)\end{array}$ \\
\hline \multicolumn{2}{|l|}{ Gender } \\
\hline Male & 49.5 \\
\hline Female & 50.9 \\
\hline \multicolumn{2}{|l|}{ Level of Training ${ }^{* * *}$} \\
\hline PGY-1 & 45.3 \\
\hline PGY-2 & 52.1 \\
\hline PGY-3 & 55.0 \\
\hline PGY-4+ & 26.2 \\
\hline \multicolumn{2}{|l|}{ Specialty Group ${ }^{* *}$} \\
\hline Medical & 51.3 \\
\hline Surgical & 40.3 \\
\hline Hospital-based & 44.7 \\
\hline \multicolumn{2}{|l|}{ CLE Characteristics $^{\mathrm{b}}$} \\
\hline \multicolumn{2}{|l|}{ Region $^{c * * *}$} \\
\hline Northeast & 43.9 \\
\hline Midwest & 58.8 \\
\hline South & 52.1 \\
\hline West & 56.1 \\
\hline \multicolumn{2}{|l|}{ Bed Size ${ }^{* \star *}$} \\
\hline$<200$ & 43.3 \\
\hline $200-299$ & 47.6 \\
\hline $300-399$ & 56.9 \\
\hline $400-499$ & 59.0 \\
\hline 500 or more & 56.3 \\
\hline \multicolumn{2}{|l|}{ Type of Ownership*** } \\
\hline Nongovernment, not-for-profit & 54.5 \\
\hline Investor-owned, for-profit & 50.9 \\
\hline Government, federal & 47.1 \\
\hline Government, nonfederal & 34.8 \\
\hline
\end{tabular}

DISTRIBUTION ACROSS CLES ${ }^{d}(n=268)$

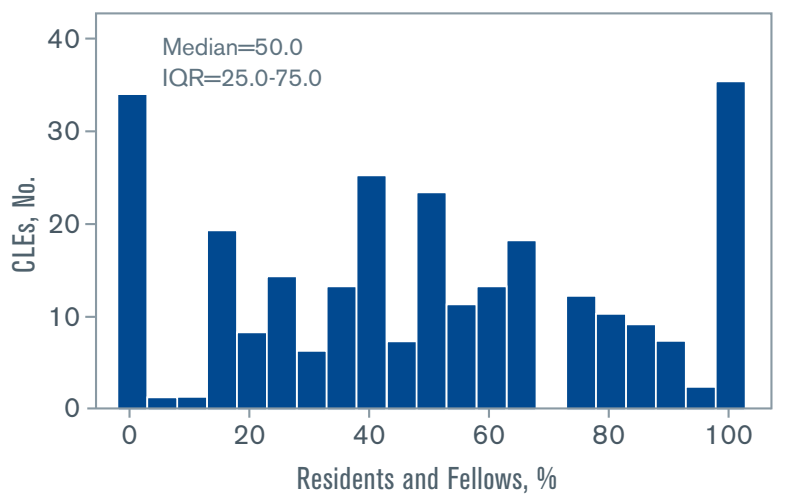

${ }^{a}$ Missing data $(<1 \%)$ have been omitted; percentages based on valid percent.

${ }^{\mathrm{b}}$ Missing data $(<7 \%)$ have been omitted; percentages based on valid percent. Of note, data are missing largely due to clinical sites that do not report data to the American Hospital Association.

${ }^{\mathrm{C}}$ Results from clinical learning environments (CLEs) in Puerto Rico (3\%) omitted to ensure anonymity.

${ }^{\mathrm{d}}$ Distribution includes $95 \%$ or more of the total number of CLEs $(\mathrm{N}=270)$.

* Statistically significant at $P<.05$.

** Statistically significant at $P<.01$.

*** Statistically significant at $P<.001$.

Abbreviation: PGY, postgraduate year. 


\section{B21. Percentage of Residents and Fellows Who Reported Knowing What They Were Allowed to Do Without Direct Supervision}

PERCENT OF TOTAL

SURVEYED ${ }^{a}(\mathrm{~N}=1837)$

\section{0}

\section{PERCENTAGE BY RESIDENT AND \\ FELLOW AND CLE CHARACTERISTICS}

\begin{tabular}{|l|c|}
\hline $\begin{array}{l}\text { Resident and Fellow } \\
\text { Characteristics }^{a}\end{array}$ & $\begin{array}{c}\text { Residents and Fellows, } \\
\%(n=1837)\end{array}$ \\
\hline \begin{tabular}{|l} 
Gender* $^{*}$ \\
Male
\end{tabular} & 96.1 \\
\hline Female & 97.8 \\
\hline Level of Training*** & \\
\hline PGY-1 & 87.5 \\
\hline PGY-2 & 97.1 \\
\hline PGY-3 & 97.3 \\
\hline PGY-4+ & 98.0 \\
\hline Specialty Group & \\
\hline Medical & 97.1 \\
\hline Surgical & 99.2 \\
\hline Hospital-based & 92.7 \\
\hline CLE Characteristics & \\
\hline
\end{tabular}

\section{Region ${ }^{c}$}

Northeast

97.4

Midwest

South

West

97.7

95.4

97.6

\section{Bed Size ${ }^{* * *}$}

$$
<200
$$

200-299

300-399

400-499

500 or more

98.3

98.3

97.5

93.0

96.2

\section{Type of Ownership**}

Nongovernment, not-for-profit

96.9

Investor-owned, for-profit

94.2

Government, federal 100

Government, nonfederal

\footnotetext{
${ }^{a}$ Missing data $(<1 \%)$ have been omitted; percentages based on valid percent.

${ }^{\mathrm{b}}$ Missing data $(<7 \%)$ have been omitted; percentages based on valid percent. Of note, data are missing largely due to clinical sites that do not report data to the American Hospital Association.

${ }^{\circ}$ Results from clinical learning environments (CLEs) in Puerto Rico (3\%) omitted to ensure anonymity.

${ }^{d}$ Distribution includes $95 \%$ or more of the total number of CLEs $(\mathrm{N}=270)$.

* Statistically significant at $P<.05$.

** Statistically significant at $P<.01$.

*** Statistically significant at $P<.001$.

Abbreviation: PGY, postgraduate year.
} 


\section{B22. Percentage of Residents and Fellows Who Reported Having an Objective Way to Know What Procedures Residents and Fellows From Other Services Were Allowed to Do Without Direct Supervision When They Consulted on Patients}

PERCENT OF TOTAL

SURVEYED ${ }^{a}(\mathrm{~N}=1695)$

\section{6}

\section{PERCENTAGE BY RESIDENT AND \\ FELLOW AND CLE CHARACTERISTICS}

\begin{tabular}{|c|c|}
\hline $\begin{array}{l}\text { Resident and Fellow } \\
\text { Characteristics }^{\mathrm{a}}\end{array}$ & $\begin{array}{l}\text { Residents and Fellows } \\
\%(\mathrm{n}=1695)\end{array}$ \\
\hline \multicolumn{2}{|l|}{ Gender } \\
\hline Male & 40.5 \\
\hline Female & 38.9 \\
\hline \multicolumn{2}{|l|}{ Level of Training*** } \\
\hline PGY-1 & 29.7 \\
\hline PGY-2 & 38.0 \\
\hline PGY-3 & 38.7 \\
\hline PGY-4+ & 53.8 \\
\hline \multicolumn{2}{|l|}{ Specialty Group*** } \\
\hline Medical & 38.6 \\
\hline Surgical & 60.7 \\
\hline Hospital-based & 32.8 \\
\hline \multicolumn{2}{|l|}{ CLE Characteristics $^{\mathrm{b}}$} \\
\hline \multicolumn{2}{|l|}{ Region $^{c * * *}$} \\
\hline Northeast & 46.7 \\
\hline Midwest & 39.3 \\
\hline South & 35.2 \\
\hline West & 34.7 \\
\hline \multicolumn{2}{|l|}{ Bed Size* } \\
\hline$<200$ & 43.1 \\
\hline $200-299$ & 37.5 \\
\hline 300-399 & 42.1 \\
\hline $400-499$ & 38.6 \\
\hline 500 or more & 35.4 \\
\hline \multicolumn{2}{|l|}{ Type of Ownership*** } \\
\hline Nongovernment, not-for-profit & 37.5 \\
\hline Investor-owned, for-profit & 42.4 \\
\hline Government, federal & 42.6 \\
\hline Government, nonfederal & 50.5 \\
\hline
\end{tabular}

DISTRIBUTION ACROSS CLEs ${ }^{d}(n=244)$

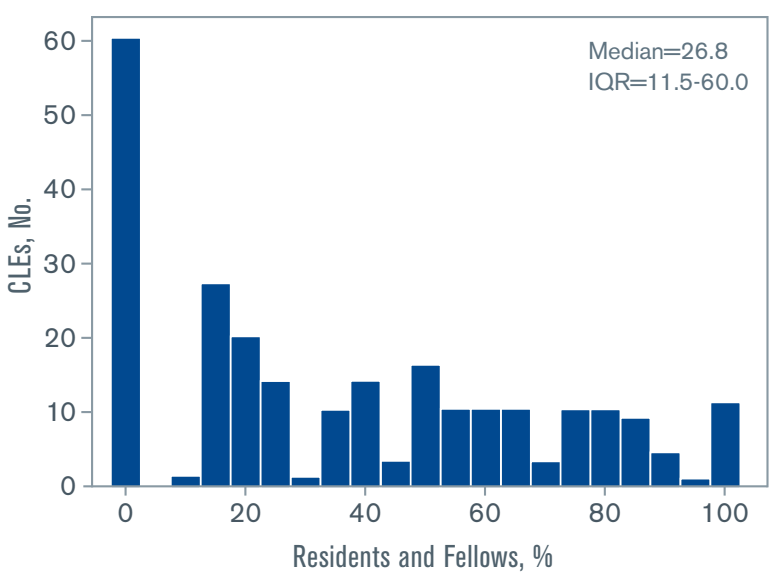

a Missing data $(<1 \%)$ have been omitted; percentages based on valid percent.

${ }^{\mathrm{b}}$ Missing data $(<2 \%)$ have been omitted; percentages based on valid percent. Of note, data are missing largely due to clinical sites that do not report data to the American Hospital Association.

${ }^{c}$ Results from clinical learning environments (CLEs) in Puerto Rico (3\%) omitted to ensure anonymity.

${ }^{\mathrm{d}}$ Distribution includes $95 \%$ or more of the total number of CLEs $(\mathrm{N}=246)$. Of note, results from visits that were held exclusively in the ambulatory care setting (9\%) have been omitted due to modifications to the question and/or scenario to better fit the setting; these modifications limited comparability across inpatient and ambulatory care settings.

* Statistically significant at $P<.05$.

** Statistically significant at $P<.01$.

*** Statistically significant at $P<.001$.

Abbreviation: PGY, postgraduate year. 


\section{B23. Percentage of Residents and Fellows Who Reported That They Would Power Through to Handoff or the End of the Day if Placed in a Situation in Which They Were Impaired by Fatigue, They Were Maxed Out on Caffeine, and a 15-Minute Nap Had No Effect}

PERCENT OF TOTAL

SURVEYED ${ }^{a}(\mathrm{~N}=1838)$

\section{0}

\section{PERCENTAGE BY RESIDENT AND \\ FELLOW AND CLE CHARACTERISTICS}

\begin{tabular}{|c|c|}
\hline $\begin{array}{l}\text { Resident and Fellow } \\
\text { Characteristics }^{\mathrm{a}}\end{array}$ & $\begin{array}{c}\text { Residents and Fellows, } \\
\qquad \%(n=1838)\end{array}$ \\
\hline \multicolumn{2}{|l|}{ Gender** } \\
\hline Male & 29.7 \\
\hline Female & 36.4 \\
\hline \multicolumn{2}{|l|}{ Level of Training*** } \\
\hline PGY-1 & 45.3 \\
\hline PGY-2 & 32.8 \\
\hline PGY-3 & 35.7 \\
\hline PGY-4+ & 20.0 \\
\hline \multicolumn{2}{|l|}{ Specialty Group* } \\
\hline Medical & 33.1 \\
\hline Surgical & 28.6 \\
\hline Hospital-based & 35.8 \\
\hline \multicolumn{2}{|l|}{ CLE Characteristics $^{\mathrm{b}}$} \\
\hline \multicolumn{2}{|l|}{ Region ${ }^{*}$} \\
\hline Northeast & 31.8 \\
\hline Midwest & 29.4 \\
\hline South & 32.5 \\
\hline West & 37.0 \\
\hline \multicolumn{2}{|l|}{ Bed Size ${ }^{* * *}$} \\
\hline$<200$ & 25.8 \\
\hline $200-299$ & 32.3 \\
\hline 300-399 & 37.7 \\
\hline $400-499$ & 37.1 \\
\hline 500 or more & 31.9 \\
\hline \multicolumn{2}{|l|}{ Type of Ownership* } \\
\hline Nongovernment, not-for-profit & 33.4 \\
\hline Investor-owned, for-profit & 29.9 \\
\hline Government, federal & 40.2 \\
\hline Government, nonfederal & 23.7 \\
\hline
\end{tabular}

DISTRIBUTION ACROSS CLES ${ }^{d}(n=268)$

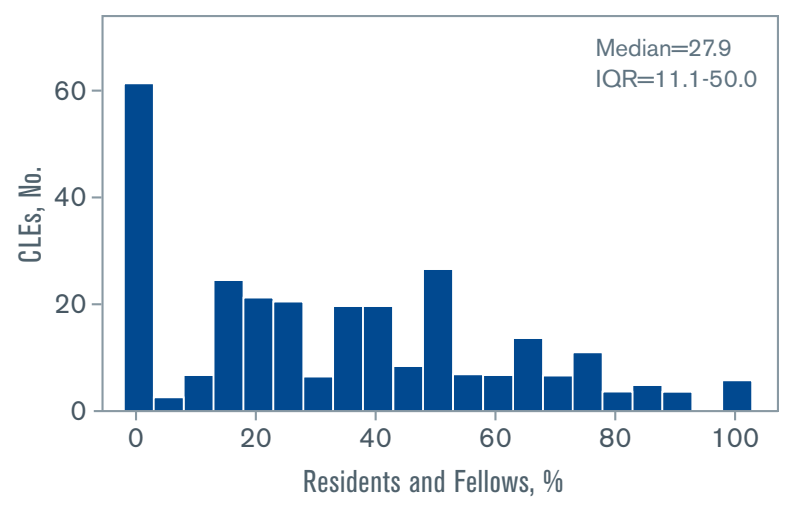

${ }^{a}$ Missing data $(<1 \%)$ have been omitted; percentages based on valid percent.

${ }^{\mathrm{b}}$ Missing data $(<7 \%)$ have been omitted; percentages based on valid percent. Of note, data are missing largely due to clinical sites that do not report data to the American Hospital Association.

${ }^{\circ}$ Results from clinical learning environments (CLEs) in Puerto Rico (3\%) omitted to ensure anonymity.

${ }^{\mathrm{d}}$ Distribution includes $95 \%$ or more of the total number of CLEs $(\mathrm{N}=270)$.

* Statistically significant at $P<.05$.

${ }^{* *}$ Statistically significant at $P<.01$.

*** Statistically significant at $P<.001$.

Abbreviation: PGY, postgraduate year. 


\section{B24. Percentage of Residents and Fellows Who Reported That Their Clinical Site Provided a Supportive, Nonpunitive Environment For Coming Forward With Concerns Regarding Honesty in Reporting (eg, Patient Data, Duty Hours)}

PERCENT OF TOTAL

SURVEYED ${ }^{a}(\mathrm{~N}=1835)$

\section{9}

\section{PERCENTAGE BY RESIDENT AND \\ FELLOW AND CLE CHARACTERISTICS}

\section{Resident and Fellow \\ Characteristics $^{\mathrm{a}}$ \\ Residents and Fellows, $\%(n=1835)$}

Gender

Male

89.3

Female

90.6

\section{Level of Training}

PGY-1

PGY-2

PGY-3

PGY-4+

Specialty Group

Medical

Surgical

Hospital-based

\section{CLE Characteristics ${ }^{\mathrm{b}}$}

\section{Region $^{\text {** }}$}

Northeast

Midwest

South

West

Bed Size

$<200$

200-299

300-399

400-499

500 or more

\section{Type of Ownership*}

Nongovernment, not-for-profit

92.2

90.0

90.0

89.1

92.1

Investor-owned, for-profit

Government, federal

Government, nonfederal
89.6

89.1

95.1
DISTRIBUTION ACROSS CLES ${ }^{d}(n=268)$

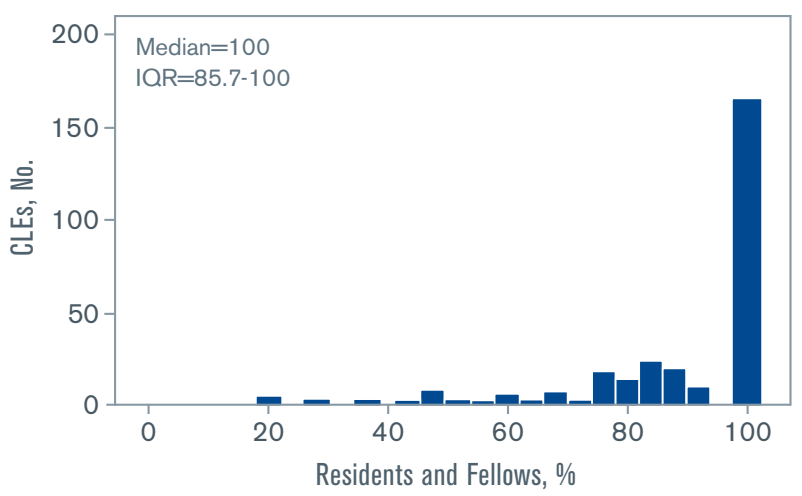

${ }^{a}$ Missing data $(<1 \%)$ have been omitted; percentages based on valid percent.

${ }^{\mathrm{b}}$ Missing data $(<7 \%)$ have been omitted; percentages based on valid percent. Of note, data are missing largely due to clinical sites that do not report data to the American Hospital Association.

${ }^{\mathrm{C}}$ Results from clinical learning environments (CLEs) in Puerto Rico (3\%) omitted to ensure anonymity.

${ }^{\mathrm{d}}$ Distribution includes $95 \%$ or more of the total number of CLEs $(\mathrm{N}=270)$.

* Statistically significant at $P<.05$.

** Statistically significant at $P<.01$.

*** Statistically significant at $P<.001$.

Abbreviation: PGY, postgraduate year. 


\section{B25. Percentage of Residents and Fellows Who Reported They Had Documented a History or Physical Finding in a Patient Medical Record That They Did Not Personally Elicit at the Clinical Site (eg, Copying and Pasting From Another Note)}

PERCENT OF TOTAL

SURVEYED ${ }^{a}(\mathrm{~N}=1830)$

\section{6}

\section{PERCENTAGE BY RESIDENT AND \\ FELLOW AND CLE CHARACTERISTICS}

\begin{tabular}{|c|c|}
\hline $\begin{array}{l}\text { Resident and Fellow } \\
\text { Characteristics }^{\mathrm{a}}\end{array}$ & $\begin{array}{c}\text { Residents and Fellows, } \\
\qquad \%(\mathrm{n}=1830)\end{array}$ \\
\hline \multicolumn{2}{|l|}{ Gender } \\
\hline Male & 25.3 \\
\hline Female & 21.7 \\
\hline \multicolumn{2}{|l|}{ Level of Training } \\
\hline PGY-1 & 23.4 \\
\hline PGY-2 & 24.4 \\
\hline PGY-3 & 23.7 \\
\hline PGY-4+ & 19.7 \\
\hline \multicolumn{2}{|l|}{ Specialty Group } \\
\hline Medical & 23.0 \\
\hline Surgical & 26.9 \\
\hline Hospital-based & 27.6 \\
\hline \multicolumn{2}{|l|}{ CLE Characteristics $^{\text {b }}$} \\
\hline \multicolumn{2}{|l|}{ Region $^{c * *}$} \\
\hline Northeast & 19.2 \\
\hline Midwest & 28.3 \\
\hline South & 22.4 \\
\hline West & 25.5 \\
\hline \multicolumn{2}{|l|}{ Bed Size } \\
\hline$<200$ & 21.6 \\
\hline $200-299$ & 20.0 \\
\hline $300-399$ & 22.4 \\
\hline $400-499$ & 25.5 \\
\hline 500 or more & 29.5 \\
\hline \multicolumn{2}{|l|}{ Type of Ownership** } \\
\hline Nongovernment, not-for-profit & 23.8 \\
\hline Investor-owned, for-profit & 20.2 \\
\hline Government, federal & 34.3 \\
\hline Government, nonfederal & 17.3 \\
\hline
\end{tabular}

DISTRIBUTION ACROSS CLES ${ }^{d}(n=268)$

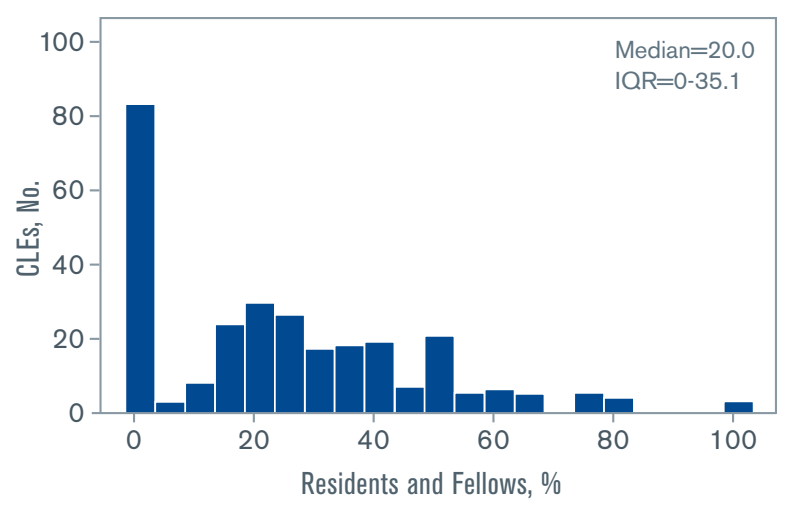

${ }^{a}$ Missing data $(<1 \%)$ have been omitted; percentages based on valid percent.

${ }^{\mathrm{b}}$ Missing data $(<7 \%)$ have been omitted; percentages based on valid percent. Of note, data are missing largely due to clinical sites that do not report data to the American Hospital Association.

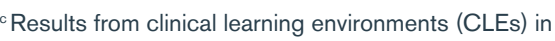
Puerto Rico (3\%) omitted to ensure anonymity.

${ }^{d}$ Distribution includes $95 \%$ or more of the total number of CLEs $(\mathrm{N}=270)$.

* Statistically significant at $P<.05$.

** Statistically significant at $P<.01$.

*** Statistically significant at $P<.001$.

Abbreviation: PGY, postgraduate year. 
B26. Percentage of Residents and Fellows Who Reported Having Felt Pressured to Compromise Their Honesty or Integrity to Satisfy an Authority Figure During Their Training at the Clinical Site

PERCENT OF TOTAL

SURVEYED ${ }^{a}(\mathrm{~N}=1835)$

\section{1}

\section{PERCENTAGE BY RESIDENT AND \\ FELLOW AND CLE CHARACTERISTICS}

\begin{tabular}{|c|c|}
\hline $\begin{array}{l}\text { Resident and Fellow } \\
\text { Characteristics }^{\mathrm{a}}\end{array}$ & $\begin{array}{l}\text { Residents and Fellows, } \\
\%(n=1835)\end{array}$ \\
\hline \multicolumn{2}{|l|}{ Gender } \\
\hline Male & 10.1 \\
\hline Female & 12.2 \\
\hline \multicolumn{2}{|l|}{ Level of Training } \\
\hline PGY-1 & 7.8 \\
\hline PGY-2 & 11.3 \\
\hline PGY-3 & 12.5 \\
\hline PGY-4+ & 6.8 \\
\hline \multicolumn{2}{|l|}{ Specialty Group } \\
\hline Medical & 11.3 \\
\hline Surgical & 12.6 \\
\hline Hospital-based & 8.1 \\
\hline \multicolumn{2}{|l|}{ CLE Characteristics $^{b}$} \\
\hline \multicolumn{2}{|l|}{ Region $^{c}$} \\
\hline Northeast & 11.3 \\
\hline Midwest & 9.9 \\
\hline South & 14.1 \\
\hline West & 8.9 \\
\hline \multicolumn{2}{|l|}{ Bed Size* } \\
\hline$<200$ & 9.3 \\
\hline $200-299$ & 14.3 \\
\hline 300-399 & 14.2 \\
\hline $400-499$ & 7.5 \\
\hline 500 or more & 9.9 \\
\hline \multicolumn{2}{|l|}{ Type of Ownership } \\
\hline Nongovernment, not-for-profit & 10.8 \\
\hline Investor-owned, for-profit & 15.7 \\
\hline Government, federal & 10.8 \\
\hline Government, nonfederal & 8.6 \\
\hline
\end{tabular}

DISTRIBUTION ACROSS CLEs ${ }^{d}(n=268)$

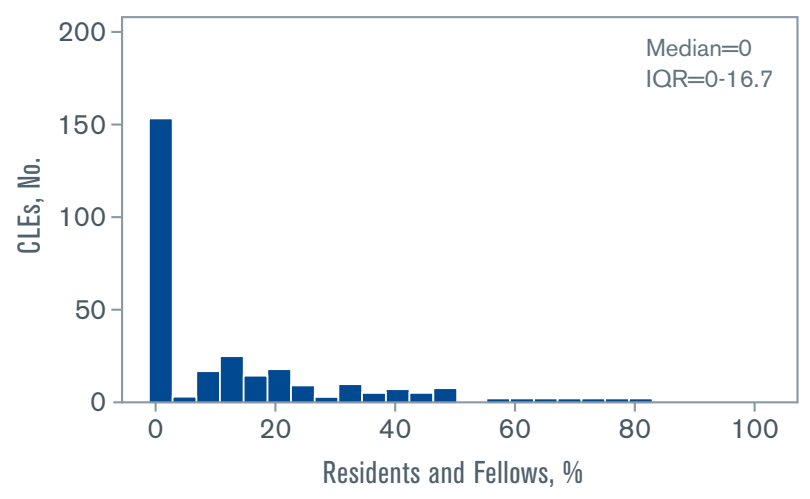

${ }^{a}$ Missing data $(<1 \%)$ have been omitted; percentages based on valid percent.

${ }^{\mathrm{b}}$ Missing data $(<7 \%)$ have been omitted; percentages based on valid percent. Of note, data are missing largely due to clinical sites that do not report data to the American Hospital Association.

${ }^{\mathrm{c}}$ Results from clinical learning environments (CLEs) in Puerto Rico $(3 \%)$ omitted to ensure anonymity.

${ }^{d}$ Distribution includes $95 \%$ or more of the total number of CLEs $(\mathrm{N}=270)$.

* Statistically significant at $P<.05$.

** Statistically significant at $P<.01$.

*** Statistically significant at $P<.001$.

Abbreviation: PGY, postgraduate year. 


\section{Appendix C. \\ SELECTED QUALITATIVE RESULTS FROM CLINICAL LEARNING ENVIRONMENT REVIEW SITE VISIT REPORTS}

\section{C1. Percentage of Clinical Learning Environments That Tracked the Number of Patient Safety Event Reports Submitted by Residents and Fellows ${ }^{a}$}

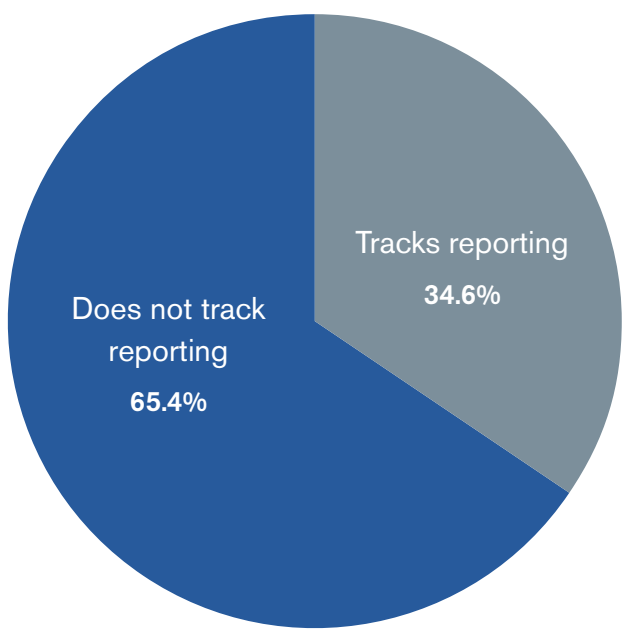

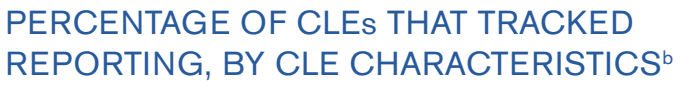

\begin{tabular}{|l|l|}
\hline Characteristics & CLEs, \% \\
\hline Region & \\
\hline Northeast & 35.0 \\
\hline Midwest & 39.7 \\
\hline South & 31.4 \\
\hline West & 32.7 \\
\hline Bed Size & \\
\hline$<200$ & 34.7 \\
\hline $200-299$ & 31.4 \\
\hline $300-399$ & 35.3 \\
\hline $400-499$ & 29.7 \\
\hline 500 or more & 41.7 \\
\hline Type of Ownership & \\
\hline Nongovernment, not-for-profit & 32.6 \\
\hline Investor-owned, for-profit & 33.3 \\
\hline Government, federal & 53.8 \\
\hline Government, nonfederal & 37.9 \\
\hline
\end{tabular}

${ }^{a}$ Missing data $(<1 \%)$ have been omitted; percentages based on valid percent. Of note, data are missing largely due to the development and refinement of a formal written site visit report template in the early stages of program implementation.

${ }^{\mathrm{b}}$ Missing data $(<8 \%)$ have been omitted; percentages based on valid percent. Of note, data are missing largely due to clinical sites that do not report data to the American Hospital Association.

${ }^{\mathrm{c}}$ Results from clinical learning environments (CLEs) in Puerto Rico (3\%) have been omitted to ensure anonymity. 


\section{C2. Percentage of Clinical Learning Environments by Proportion of Nurses' Understanding of Reportable Patient Safety Events ${ }^{2}$}
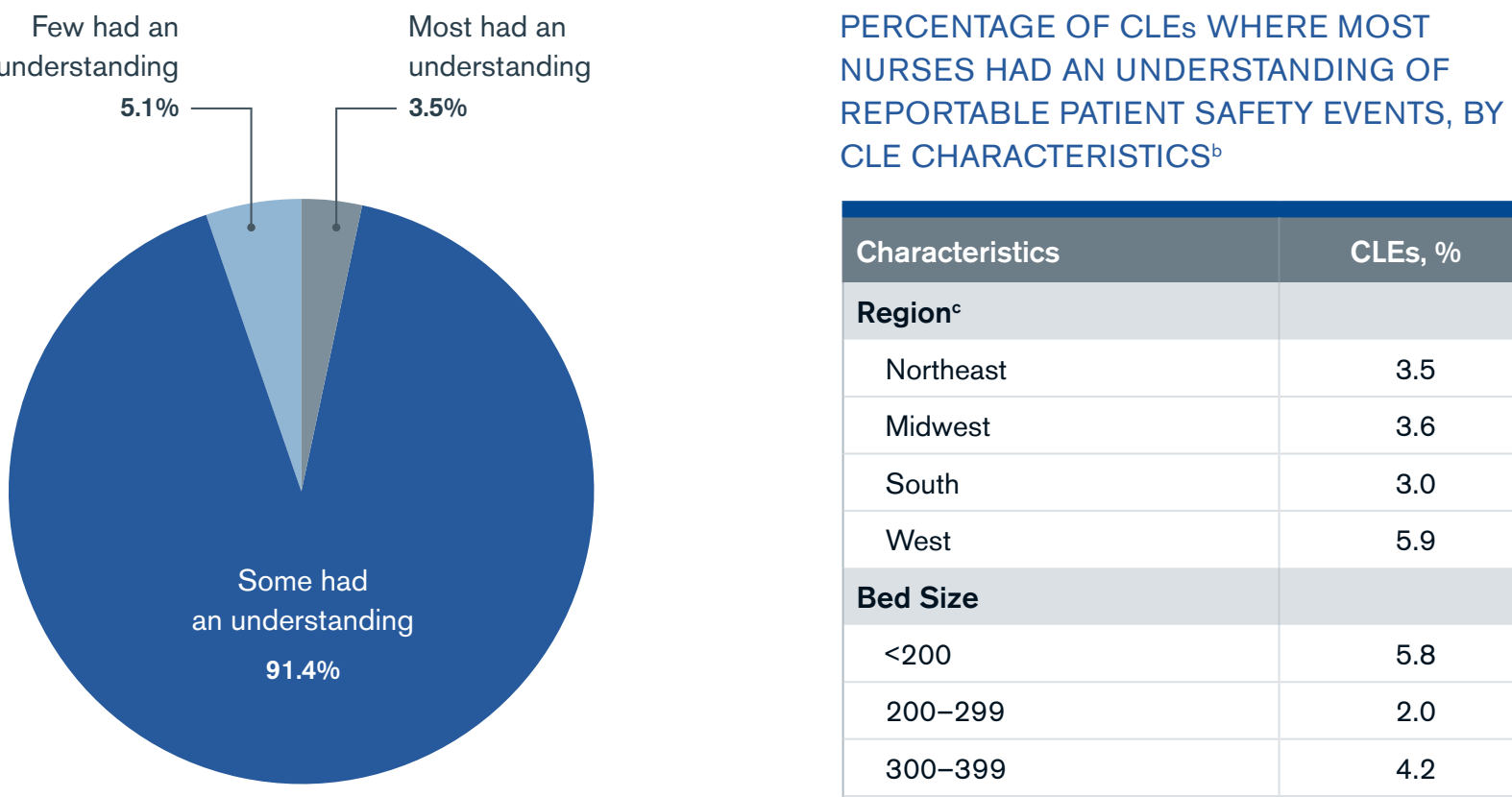

PERCENTAGE OF CLEs WHERE MOST NURSES HAD AN UNDERSTANDING OF REPORTABLE PATIENT SAFETY EVENTS, BY CLE CHARACTERISTICS

\begin{tabular}{|l|l|}
\hline Characteristics & CLEs, \% \\
\hline Region ${ }^{c}$ & \\
\hline Northeast & 3.5 \\
\hline Midwest & 3.6 \\
\hline South & 3.0 \\
\hline West & 5.9 \\
\hline Bed Size & \\
\hline$<200$ & 5.8 \\
\hline $200-299$ & 2.0 \\
\hline $300-399$ & 4.2 \\
\hline $400-499$ & 2.8 \\
\hline 500 or more & 2.9 \\
\hline Type of Ownership & \\
\hline Nongovernment, not-for-profit & 3.6 \\
\hline Investor-owned, for-profit & 0.0 \\
\hline Government, federal & 8.3 \\
\hline Government, nonfederal & 7.4 \\
\hline
\end{tabular}

\footnotetext{
${ }^{a}$ Missing data $(<6 \%)$ have been omitted; percentages based on valid percent. Of note, data are missing largely due to the development and refinement of a formal written site visit report template in the early stages of program implementation.

${ }^{\mathrm{b}}$ Missing data $(<13 \%)$ have been omitted; percentages based on valid percent. Of note, data are missing largely due to clinical sites that do not report data to the American Hospital Association.

${ }^{c}$ Results from clinical learning environments (CLEs) in Puerto Rico (3\%) have been omitted to ensure anonymity.
} 


\section{C3. Percentage of Clinical Learning Environments Where Residents and Fellows Frequently Submitted Patient Safety Event Reports ${ }^{a}$}

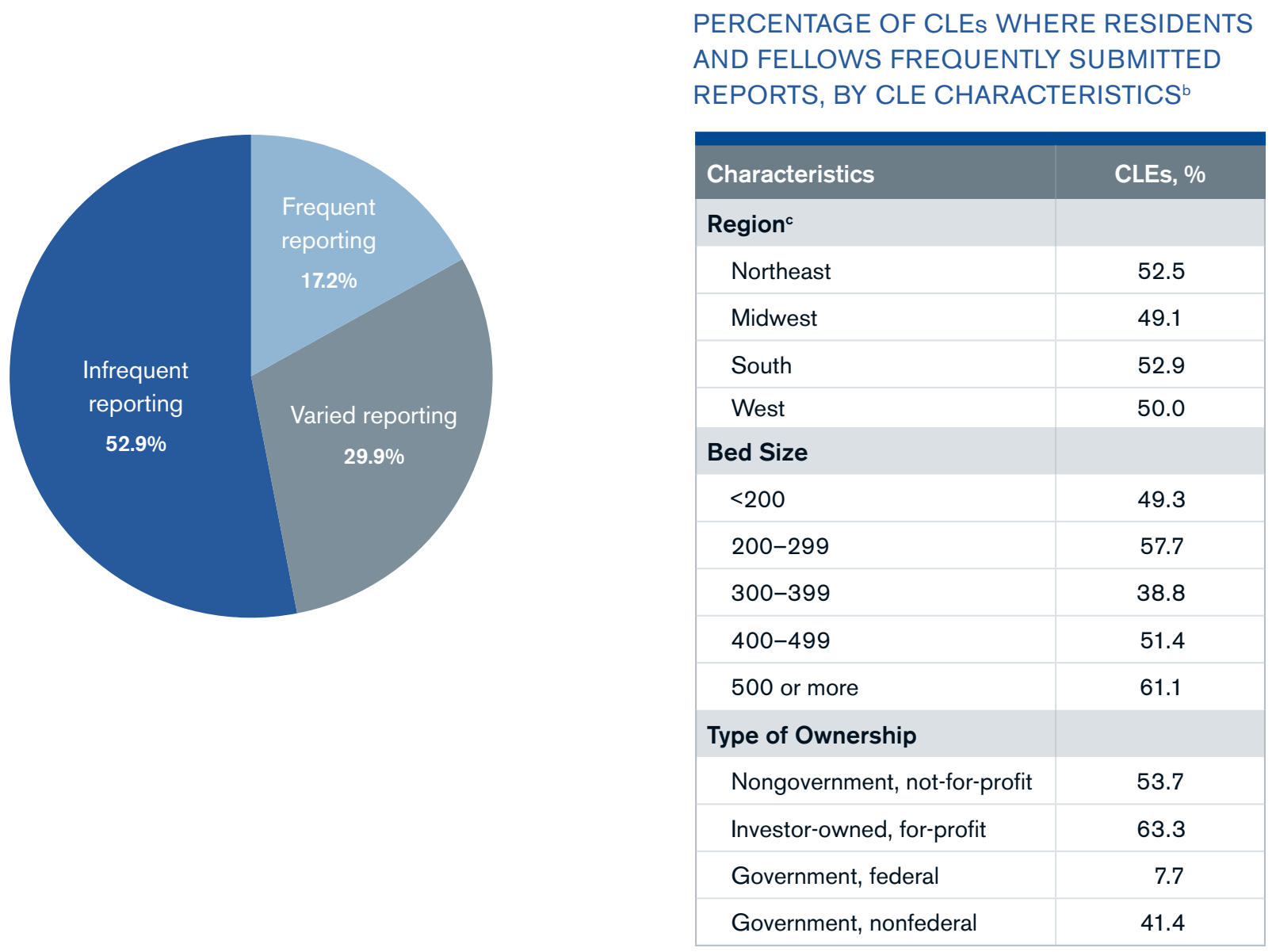

\footnotetext{
${ }^{a}$ Missing data $(<1 \%)$ have been omitted; percentages based on valid percent. Of note, data are missing largely due to the development and refinement of a formal written site visit report template in the early stages of program implementation.

${ }^{b}$ Missing data $(<8 \%)$ have been omitted; percentages based on valid percent. Of note, data are missing largely due to clinical sites that do not report data to the American Hospital Association.

${ }^{\circ}$ Results from clinical learning environments (CLEs) in Puerto Rico (3\%) have been omitted to ensure anonymity.
} 


\section{C4. Percentage of Clinical Learning Environments by Proportion of Resident and Fellow Knowledge of Basic Patient Safety Terminology and Principles ${ }^{a}$}

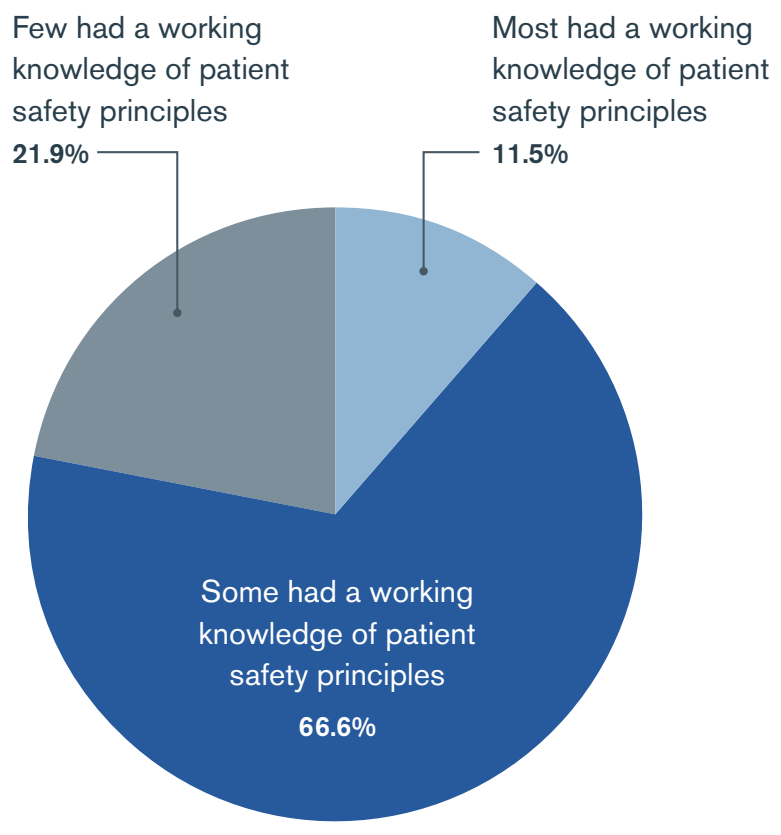

\begin{abstract}
PERCENTAGE OF CLEs WHERE MOST RESIDENTS AND FELLOWS HAD A WORKING KNOWLEDGE OF PATIENT SAFETY PRINCIPLES, BY CLE CHARACTERISTICS ${ }^{b}$
\end{abstract}

\begin{tabular}{|l|r|}
\hline Characteristics & CLEs, \% \\
\hline Region & \\
\hline Northeast & 6.6 \\
\hline Midwest & 15.5 \\
\hline South & 8.7 \\
\hline West & 12.7 \\
\hline Bed Size & \\
\hline$<200$ & 9.3 \\
\hline $200-299$ & 11.5 \\
\hline $300-399$ & 12.0 \\
\hline $400-499$ & 13.5 \\
\hline 500 or more & 5.6 \\
\hline Type of Ownership & 10.7 \\
\hline Nongovernment, not-for-profit & 9.7 \\
\hline Investor-owned, for-profit & 23.1 \\
\hline Government, federal & 3.6 \\
\hline Government, nonfederal & \\
\hline
\end{tabular}

\footnotetext{
${ }^{a}$ Missing data $(<1 \%)$ have been omitted; percentages based on valid percent. Of note, data are missing largely due to the development and refinement of a formal written site visit report template in the early stages of program implementation.

${ }^{b}$ Missing data $(<8 \%)$ have been omitted; percentages based on valid percent. Of note, data are missing largely due to clinical sites that do not report data to the American Hospital Association.

${ }^{\mathrm{c}}$ Results from clinical learning environments (CLEs) in Puerto Rico (3\%) have been omitted to ensure anonymity.
} 


\section{C5. Percentage of Clinical Learning Environments by Proportion of Resident and Fellow Knowledge of Basic Quality Improvement Concepts ${ }^{a}$}

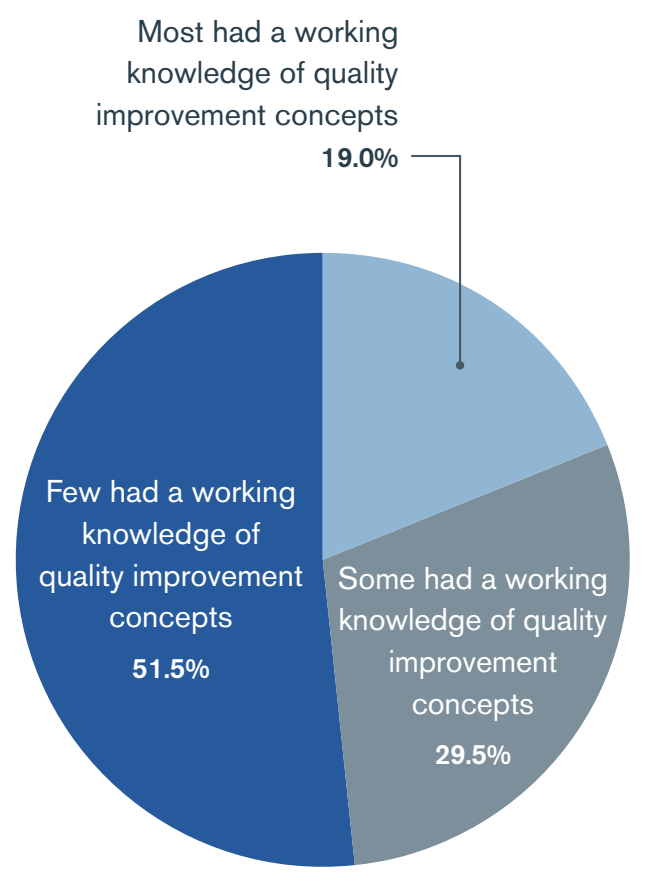
PERCENTAGE OF CLEs WHERE MOST RESIDENTS AND FELLOWS HAD A WORKING KNOWLEDGE OF QUALITY IMPROVEMENT CONCEPTS, BY CLE CHARACTERISTICS ${ }^{b}$

\begin{tabular}{|l|l|}
\hline Characteristics & CLEs, \% \\
\hline Region & \\
\hline Northeast & 18.3 \\
\hline Midwest & 15.5 \\
\hline South & 18.6 \\
\hline West & 22.2 \\
\hline Bed Size & \\
\hline$<200$ & 23.0 \\
\hline $200-299$ & 11.8 \\
\hline $300-399$ & 19.6 \\
\hline $400-499$ & 13.5 \\
\hline 500 or more & 22.2 \\
\hline Type of Ownership & \\
\hline Nongovernment, not-for-profit & 18.6 \\
\hline Investor-owned, for-profit & 12.9 \\
\hline Government, federal & 23.1 \\
\hline Government, nonfederal & 21.4 \\
\hline
\end{tabular}

\footnotetext{
${ }^{a}$ Missing data $(<1 \%)$ have been omitted; percentages based on valid percent. Of note, data are missing largely due to the development and refinement of a formal written site visit report template in the early stages of program implementation.

${ }^{\mathrm{b}}$ Missing data $(<8 \%)$ have been omitted; percentages based on valid percent. Of note, data are missing largely due to clinical sites that do not report data to the American Hospital Association.

${ }^{\circ}$ Results from clinical learning environments (CLEs) in Puerto Rico (3\%) have been omitted to ensure anonymity.
} 


\title{
C6. Percentage of Clinical Learning Environments by Proportion of Resident and Fellow Quality Improvement Projects With Components of a Complete Quality Improvement Cycle ${ }^{a}$
}

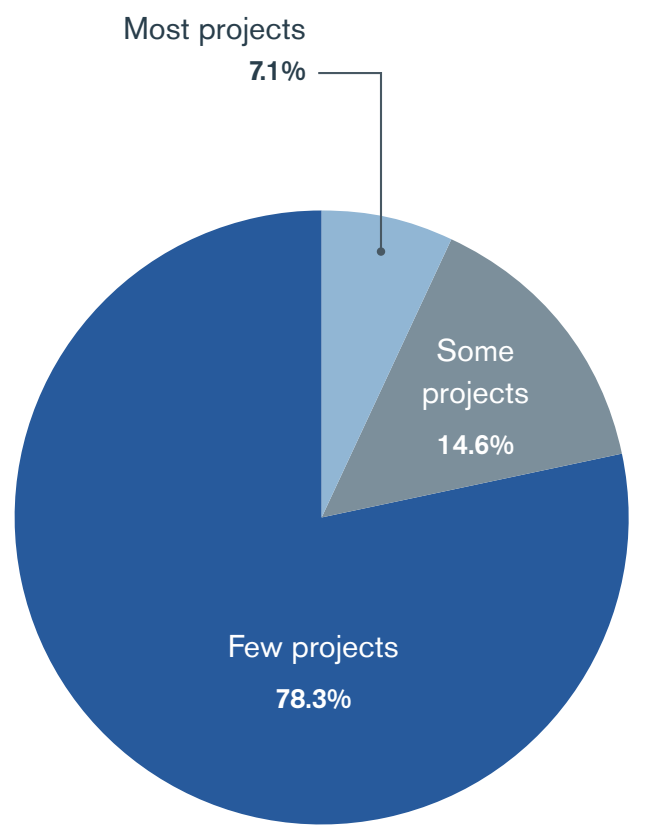

\author{
PERCENTAGE OF CLEs WHERE MOST \\ RESIDENT AND FELLOW QUALITY \\ IMPROVEMENT PROJECTS HAD COMPONENTS \\ OF A COMPLETE QUALITY IMPROVEMENT \\ CYCLE, BY CLE CHARACTERISTICS ${ }^{\text {b }}$
}

\begin{tabular}{|l|c|}
\hline Characteristics & CLEs, \% \\
\hline Region & \\
\hline Northeast & 5.0 \\
\hline Midwest & 6.9 \\
\hline South & 4.3 \\
\hline West & 11.1 \\
\hline Bed Size & \\
\hline$<200$ & 9.3 \\
\hline $200-299$ & 2.0 \\
\hline $300-399$ & 7.8 \\
\hline $400-499$ & 2.7 \\
\hline 500 or more & 8.3 \\
\hline Type of Ownership & 6.9 \\
\hline Nongovernment, not-for-profit & 6.8 \\
\hline Investor-owned, for-profit & 6.5 \\
\hline Government, federal & 0.0 \\
\hline Government, nonfederal & 6.9 \\
\hline
\end{tabular}

\footnotetext{
${ }^{a}$ Missing data $(<1 \%)$ have been omitted; percentages based on valid percent. Of note, data are missing largely due to the development and refinement of a formal written site visit report template in the early stages of program implementation.

${ }^{b}$ Missing data $(<8 \%)$ have been omitted; percentages based on valid percent. Of note, data are missing largely due to clinical sites that do not report data to the American Hospital Association.

${ }^{\mathrm{c}}$ Results from clinical learning environments (CLEs) in Puerto Rico (3\%) have been omitted to ensure anonymity.
} 


\section{C7. Percentage of Clinical Learning Environments With Handoff Processes That Were Standardized Across Programs, Based on Direct Observations ${ }^{a}$}

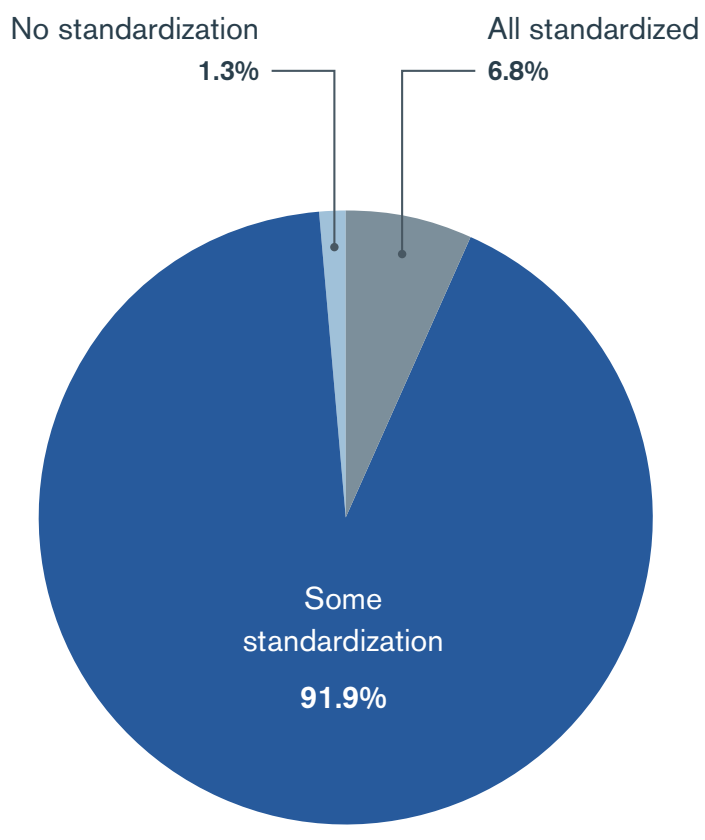
PERCENTAGE OF CLEs WITH HANDOFF PROCESSES THAT WERE ALL STANDARDIZED, BY CLE CHARACTERISTICS ${ }^{b}$

\begin{tabular}{|l|r|}
\hline Characteristics & CLEs, \% \\
\hline Region & \\
\hline Northeast & 6.6 \\
\hline Midwest & 3.9 \\
\hline South & 4.4 \\
\hline West & 10.6 \\
\hline Bed Size & \\
\hline$<200$ & 5.6 \\
\hline $200-299$ & 10.4 \\
\hline $300-399$ & 8.5 \\
\hline $400-499$ & 5.9 \\
\hline 500 or more & 2.9 \\
\hline Type of Ownership & \\
\hline Nongovernment, not-for-profit & 8.4 \\
\hline Investor-owned, for-profit & 3.7 \\
\hline Government, federal & 0.0 \\
\hline Government, nonfederal & 3.6 \\
\hline
\end{tabular}

\footnotetext{
${ }^{a}$ Missing data $(<13 \%)$ have been omitted; percentages based on valid percent. Of note, data are missing largely due to the development and refinement of a formal written site visit report template in the early stages of program implementation.

${ }^{b}$ Missing data $(<14 \%)$ have been omitted; percentages based on valid percent. Of note, data are missing largely due to clinical sites that do not report data to the American Hospital Association.

${ }^{\circ}$ Results from clinical learning environments (CLEs) in Puerto Rico (3\%) have been omitted to ensure anonymity.
} 


\section{C8. Percentage of Clinical Learning Environments by Mechanism Used for Identification of Resident and Fellow Competency to Perform Clinical Procedures, as Reported by Nurses ${ }^{a}$}

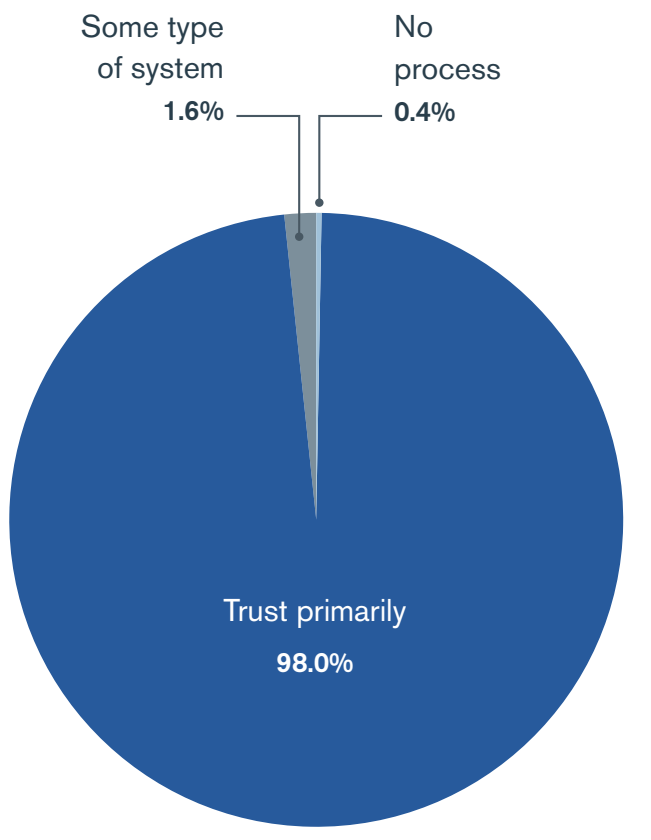

\footnotetext{
${ }^{a}$ Missing data $(<5 \%)$ have been omitted; percentages based on valid percent. Of note, data are missing largely due to the development and refinement of a formal written site visit report template in the early stages of program implementation.

${ }^{b}$ Missing data $(<11 \%)$ have been omitted; percentages based on valid percent. Of note, data are missing largely due to clinical sites that do not report data to the American Hospital Association.

${ }^{\circ}$ Results from clinical learning environments (CLEs) in Puerto Rico (3\%) have been omitted to ensure anonymity.
} 


\section{C9. Percentage of Clinical Learning Environments Where Patient Safety and Quality Leaders Recalled Patient Safety Events Related to Resident and Fellow Fatigue}
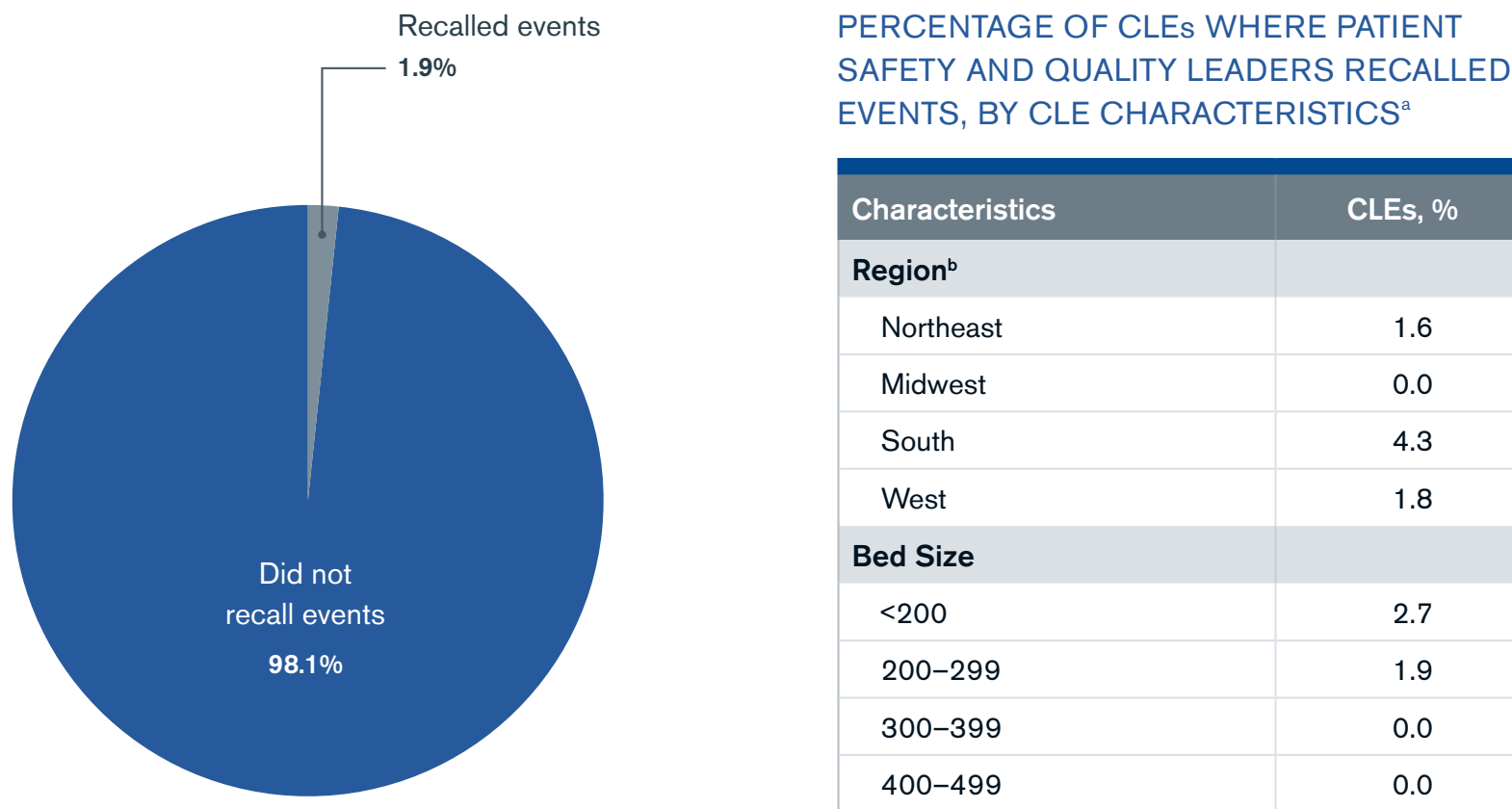

\begin{tabular}{|l|c|}
\hline Characteristics & CLEs, \% \\
\hline Region ${ }^{\text {b }}$ & 1.6 \\
\hline Northeast & 0.0 \\
\hline Midwest & 4.3 \\
\hline South & 1.8 \\
\hline West & \\
\hline Bed Size & 2.7 \\
\hline <200 & 1.9 \\
\hline $200-299$ & 0.0 \\
\hline 300-399 & 0.0 \\
\hline $400-499$ & 5.6 \\
\hline 500 or more & \\
\hline Type of Ownership & 1.7 \\
\hline Nongovernment, not-for-profit & 0.0 \\
\hline Investor-owned, for-profit & 15.4 \\
\hline Government, federal & 0.0 \\
\hline Government, nonfederal & \\
\hline
\end{tabular}

\footnotetext{
${ }^{a}$ Missing data $(<8 \%)$ have been omitted; percentages based on valid percent. Of note, data are missing largely due to clinical sites that do not report data to the American Hospital Association.

${ }^{\mathrm{b}}$ Results from clinical learning environments (CLEs) in Puerto Rico (3\%) have been omitted to ensure anonymity.
} 


\section{C10. Percentage of Clinical Learning Environments Where Residents and Fellows Reported Observing Some Signs of Burnout Among Faculty Members and Program Directors ${ }^{a}$}

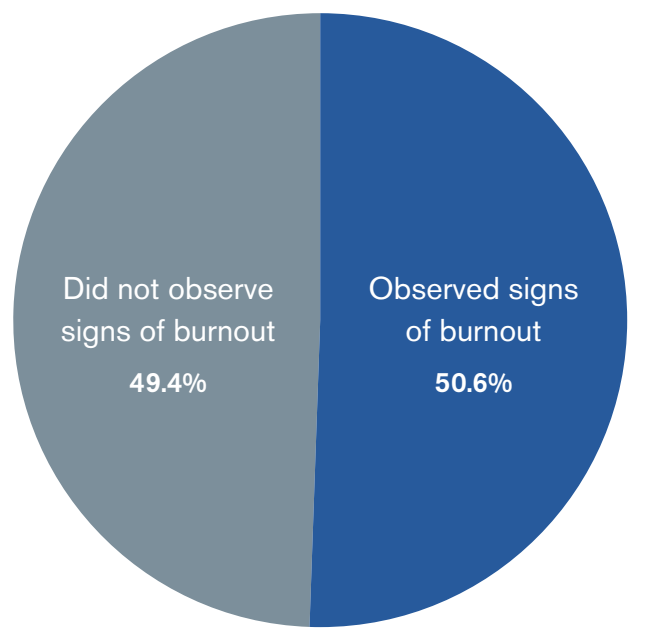

\section{PERCENTAGE OF CLES WHERE RESIDENTS AND FELLOWS REPORTED OBSERVING SIGNS} OF BURNOUT, BY CLE CHARACTERISTICS ${ }^{\mathrm{b}}$

\begin{tabular}{|l|l|}
\hline Characteristics & CLEs, \% \\
\hline Region & \\
\hline Northeast & 51.7 \\
\hline Midwest & 50.9 \\
\hline South & 41.5 \\
\hline West & 60.8 \\
\hline Bed Size & \\
\hline$<200$ & 39.7 \\
\hline $200-299$ & 50.0 \\
\hline $300-399$ & 58.0 \\
\hline $400-499$ & 54.5 \\
\hline 500 or more & 55.9 \\
\hline Type of Ownership & \\
\hline Nongovernment, not-for-profit & 53.0 \\
\hline Investor-owned, for-profit & 42.9 \\
\hline Government, federal & 46.2 \\
\hline Government, nonfederal & 40.7 \\
\hline
\end{tabular}

\footnotetext{
${ }^{a}$ Missing data $(<7 \%)$ have been omitted; percentages based on valid percent. Of note, data are missing largely due to the development and refinement of a formal written site visit report template in the early stages of program implementation.

${ }^{b}$ Missing data $(<13 \%)$ have been omitted; percentages based on valid percent. Of note, data are missing largely due to clinical sites that do not report data to the American Hospital Association.

${ }^{\circ}$ Results from clinical learning environments (CLEs) in Puerto Rico (3\%) have been omitted to ensure anonymity.
} 


\section{C11. Percentage of Clinical Learning Environments Where Executive Leadership Recalled Incidents Concerning Professionalism}
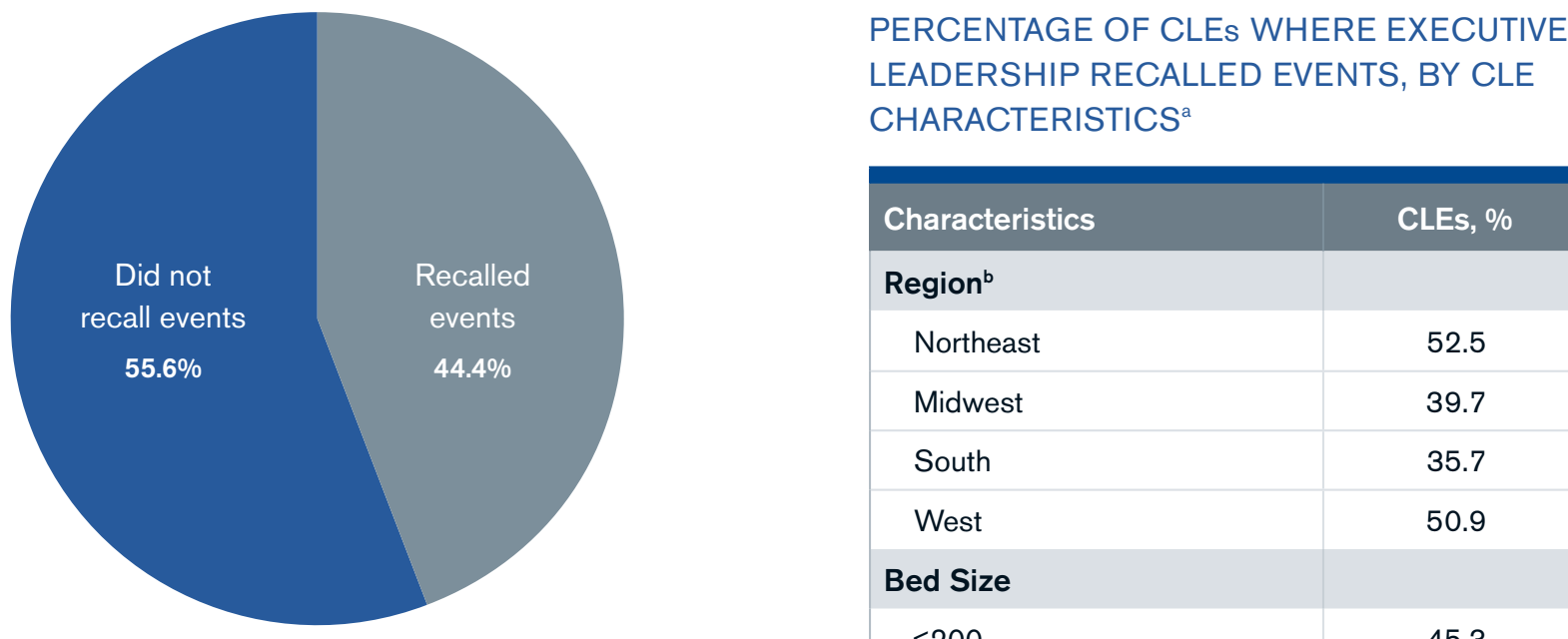

CHARACTERISTICS ${ }^{a}$

\begin{tabular}{|l|l|}
\hline Characteristics & CLEs, \% \\
\hline Region & \\
\hline Northeast & 52.5 \\
\hline Midwest & 39.7 \\
\hline South & 35.7 \\
\hline West & 50.9 \\
\hline Bed Size & \\
\hline$<200$ & 45.3 \\
\hline $200-299$ & 40.4 \\
\hline $300-399$ & 51.0 \\
\hline $400-499$ & 45.9 \\
\hline 500 or more & 33.3 \\
\hline Type of Ownership & \\
\hline Nongovernment, not-for-profit & 37.6 \\
\hline Investor-owned, for-profit & 54.8 \\
\hline Government, federal & 76.9 \\
\hline Government, nonfederal & 55.2 \\
\hline
\end{tabular}

\footnotetext{
${ }^{a}$ Missing data $(<8 \%)$ have been omitted; percentages based on valid percent. Of note, data are missing largely due to clinical sites that do not report data to the American Hospital Association.

${ }^{\mathrm{b}}$ Results from clinical learning environments (CLEs) in Puerto Rico (3\%) have been omitted to ensure anonymity.
} 


\section{C12. Percentage of Clinical Learning Environments With Reports of Chronic Disrespectful or Disruptive Behavior Across More Than 1 Clinical Unita}

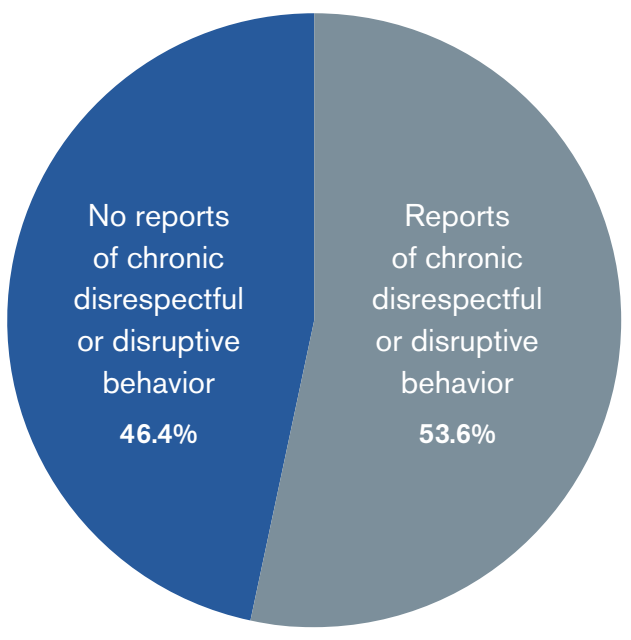
PERCENTAGE OF CLEs WITH REPORTS OF CHRONIC DISRESPECTFUL OR DISRUPTIVE BEHAVIOR, BY CLE CHARACTERISTICS ${ }^{\text {b }}$

\begin{tabular}{|l|l|}
\hline Characteristics & CLEs, \% \\
\hline Region & \\
\hline Northeast & 58.3 \\
\hline Midwest & 70.2 \\
\hline South & 49.3 \\
\hline West & 52.7 \\
\hline Bed Size & \\
\hline$<200$ & 47.3 \\
\hline $200-299$ & 57.7 \\
\hline $300-399$ & 64.0 \\
\hline $400-499$ & 63.9 \\
\hline 500 or more & 55.6 \\
\hline Type of Ownership & \\
\hline Nongovernment, not-for-profit & 61.6 \\
\hline Investor-owned, for-profit & 60.0 \\
\hline Government, federal & 23.1 \\
\hline Government, nonfederal & 35.7 \\
\hline
\end{tabular}

\footnotetext{
${ }^{a}$ Missing data $(<2 \%)$ have been omitted; percentages based on valid percent. Of note, data are missing largely due to the development and refinement of a formal written site visit report template in the early stages of program implementation.

${ }^{b}$ Missing data $(<9 \%)$ have been omitted; percentages based on valid percent. Of note, data are missing largely due to clinical sites that do not report data to the American Hospital Association.

${ }^{\circ}$ Results from clinical learning environments (CLEs) in Puerto Rico (3\%) have been omitted to ensure anonymity.
} 


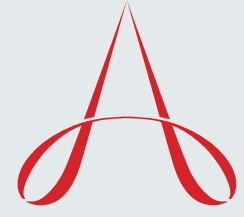

A C G M E

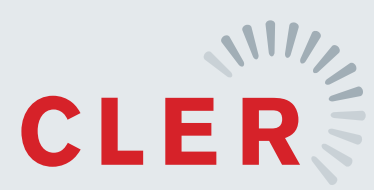

Clinical Learning Environment Review

Accreditation Council for

Graduate Medical Education

Access the full CLER National Report of Findings 2019: Initial Visits to Sponsoring Institutions With 2 or Fewer Core Residency Programs at https://doi.org/10.35425/ACGME.0001 